\title{
New avenues towards mobile brain computer interfaces
}

Citation for published version (APA):

Lührs, M. (2018). New avenues towards mobile brain computer interfaces: the impact of real-time and fast fMRI. [Doctoral Thesis, Maastricht University]. Maastricht University. https://doi.org/10.26481/dis.20180927ml

Document status and date:

Published: 01/01/2018

DOI:

$10.26481 /$ dis.20180927ml

Document Version:

Publisher's PDF, also known as Version of record

\section{Please check the document version of this publication:}

- A submitted manuscript is the version of the article upon submission and before peer-review. There can be important differences between the submitted version and the official published version of record.

People interested in the research are advised to contact the author for the final version of the publication, or visit the DOI to the publisher's website.

- The final author version and the galley proof are versions of the publication after peer review.

- The final published version features the final layout of the paper including the volume, issue and page numbers.

Link to publication

\footnotetext{
General rights rights.

- You may freely distribute the URL identifying the publication in the public portal. please follow below link for the End User Agreement:

www.umlib.nl/taverne-license

Take down policy

If you believe that this document breaches copyright please contact us at:

repository@maastrichtuniversity.nl

providing details and we will investigate your claim.
}

Copyright and moral rights for the publications made accessible in the public portal are retained by the authors and/or other copyright owners and it is a condition of accessing publications that users recognise and abide by the legal requirements associated with these

- Users may download and print one copy of any publication from the public portal for the purpose of private study or research.

- You may not further distribute the material or use it for any profit-making activity or commercial gain

If the publication is distributed under the terms of Article $25 \mathrm{fa}$ of the Dutch Copyright Act, indicated by the "Taverne" license above, 
New avenues towards mobile brain computer interfaces: the impact of real-time and fast fMRI

Michael Lührs 


\section{(c) Michael Lührs, Maastricht 2018}

All rights reserved. No part of this thesis may be reproduced, stored in a retrieval system, or transmitted in any form, or by any means, electronic, mechanical, photocopying, recording or otherwise, without prior written permission of the author.

Printed by: printclub Aachen

Cover and chapter illustrations: fabioberti.it ISBN: 978-90-829118-1-7

The work presented in this thesis was funded by the European Community's Seventh Framework Programme FP7/2007-2013 under grant agreement number PITN-GA-2011-290011 and by the European Commission's Health Cooperation Work Programme of the 7th Framework Programme, under the Grant Agreement $n^{\circ} 602186$ (BRAINTRAIN). 
New avenues towards mobile brain computer interfaces: the impact of real-time and fast fMRI

\author{
Dissertation \\ to obtain the degree of Doctor at the Maastricht University, \\ on the authority of the Rector Magnificus Prof.dr. Rianne M. Letschert \\ in accordance with the decision of the Board of Deans, \\ to be defended in public on Thursday $27^{\text {th }}$ of September 2018 at 12:00 hours \\ by
}

Michael Lührs 


\section{Supervisor}

Prof. dr. Rainer. Goebel

\section{Co-supervisor}

Dr. Fabrizio Esposito

Dr. Bettina Sorger

\section{Assessment Committee}

Prof. dr. D.E.J. Linden (Chair)

Prof. dr. M. Castelo-Branco (University of Coimbra, PRT)

Dr. J.C. Peters

Prof. dr. N. Weiskopf (Leipzig University, GER) 


\section{CONTENTS}

CHAPTER 1

General introduction

\section{CHAPTER 2}

Automated selection of brain regions for real-time fMRI Brain Computer Interfaces

CHAPTER 3

65

MR-Encephalography for $\mathrm{BCl} /$ neurofeedback applications with high temporal resolution sampling

\section{CHAPTER 4}

107

Turbo-Satori - A neurofeedback and BCI toolbox for real-time fNIRS

\section{CHAPTER 5}

Summary, general discussion and conclusion

\section{ADDENDUM}

Knowledge valorization, Acknowledgments,

Publications

\section{9}

Curriculum Vitae, 


$$
.5
$$




\section{Chapter 1}

General introduction 
In the last decades, several technological developments in neuroscience research have opened up new opportunities in the field of cognitive neuroscience. Beginning with the use of the first computers in a clinical environment which culminated in digital imaging modalities allowing in vivo diagnosis and research of the human brain. This enabled clinicians and researchers a deeper look into the human brain which was not possible before. Since the introduction of the first electrophysiological techniques like electroencephalography (EEG) in 1875 (La Vaque, 1999), neuroimaging methods of the brain got more and more attention after the development of functional magnetic resonance imaging (fMRI) in 1990 (Seiji Ogawa, Lee, Nayak, \& Glynn, 1990). fMRI allows to noninvasively investigate the human brain with a spatial resolution that was not possible before. Even though fMRI, compared to EEG, measures metabolic changes (blood flow) in the brain caused by neural activity, it provides clear insights of the human brain because of the hemodynamic coupling (Logothetis \& Pfeuffer, 2004), which describes the delivery of blood to actively used neuronal tissue. Also, a method called functional near-infrared spectroscopy (fNIRS) which, similar to fMRI, also measures metabolic changes due to brain activity was invented in 1977 even a couple of years earlier (Feinberg \& Yacoub, 2012; Ferrari, Giannini, Sideri, \& Zanette, 1985; Jöbsis, 1977; Schlereth, Fossaceca, Keckler, \& Barbour, n.d.; Torricelli et al., 2014; Wald, 2012). Using near-infrared light, it is possible to measure the differences in oxygenated and deoxygenated hemoglobin which is similarly also the basis signal for fMRI. It is based on the light absorption property of hemoglobin using at least two different wavelengths, allowing to indirectly measure differences in neuronal activity.

Further technical developments in computer science led to an increase in processing performance which paved the way for real-time analysis of $\mathrm{fMRI}$ data (rt-fMRI) at the end of the 20th century (Bagarinao, Nakai, \& Tanaka, 2006). With this new technique, several new application possibilities emerged, building the bases for, for example, new quality control measures in $\mathrm{fMRI}$ experiments as well as new experimental and therapeutic designs - the latter meant to help patients suffering from specific mental diseases in their respective recovery process (Sulzer et al., 2013; Wang, Mantini, \& Gillebert, 2017; Weiskopf, 2012). While the first publications focused on the general feasibility of analyzing $\mathrm{fMRI}$ data in real-time (DeCharms et al., 2004; Posse et al., 2000, 2003; Voyvodic, 
1999; S.-S. Yoo \& Jolesz, 2002), the focus shifted quickly towards real-time applications of computationally extensive methods like independent component analysis (ICA) and brain-computer interfacing (BCl) (Birbaumer, Murguialday, \& Cohen, 2008; Esposito et al., 2003; Yoo et al., 2004). At the same time, also fNIRS got more attention in neuroscientific research (Calderon-Arnulphi, Alaraj, \& Slavin, 2009; Lloyd-Fox, Blasi, \& Elwell, 2010). Also, here the first occurrences of real-time applications can be found in the mid-nineties of the last century, but it took more than ten years to see a significant increase in the amount of publications (see Figure 1).

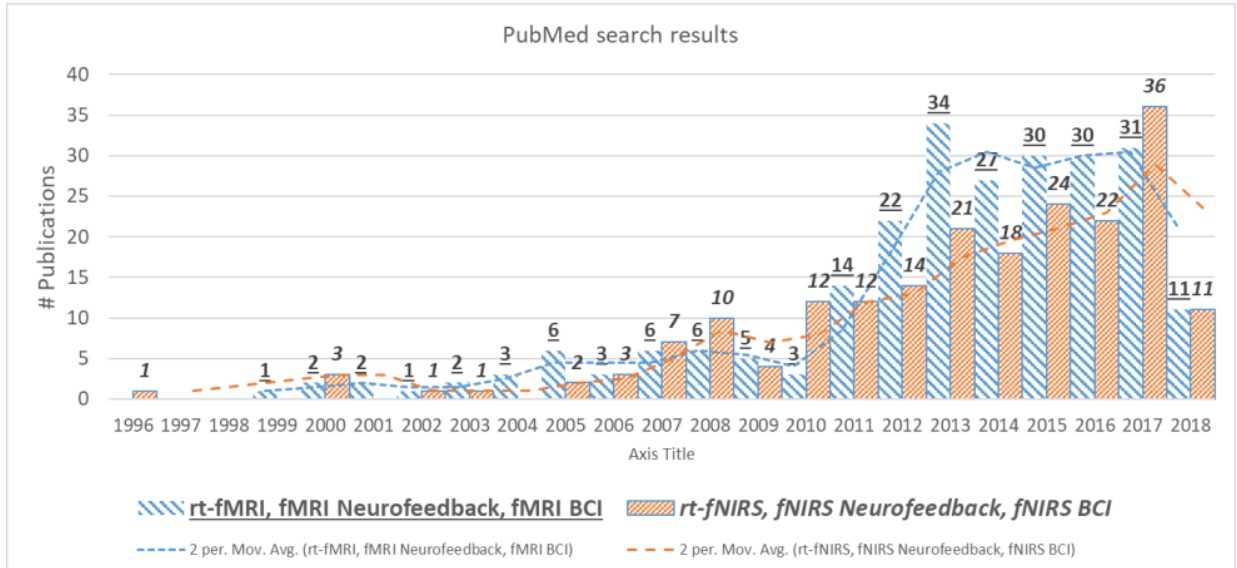

Figure 1: Number of publications based on PubMed (Kidd et al., 1999) search. PubMed is an online repository covering about 27 million citations of research articles from live science journals and books. The performed search used the following keywords: (real-time fMRI) OR (fMRI Neurofeedback) OR (fMRI BCI) for the fMRI publications and (real-time functional near-infrared spectroscopy) $O R$ (functional near-infrared spectroscopy Neurofeedback) OR (functional near-infrared spectroscopy $B C I)$ for the fNIRS publications.

Overall the current amount of yearly publications for rt-fMRI and rt-fNIRS increased more than threefold compared to 2010 (see Figure 1). This clearly shows the growing attention in this particular field of research. Also, as described in recent review articles (Boas, Elwell, Ferrari, \& Taga, 2014; Naseer \& Hong, 2015; Thibault, MacPherson, Lifshitz, Roth, \& Raz, 2018), potential applications for rt-fMRI and rt-fNIRS become more and more wide spread. 


\section{BRAIN-COMPUTER INTERFACING FOR BRAIN-BASED COMMUNICATION/CONTROL AND NEUROFEEDBACK}

The possibility to analyze neuroimaging data in real-time opens up several opportunities especially in the field of $\mathrm{BCls}$. A BCl based on $\mathrm{fMRI}$ usually consists of three separate parts: (1) the MRI scanner and its console computer, which reconstructs and stores the fMRI data in real-time; (2), the real-time analysis machine which performs fMRI preprocessing and statistical analysis of the data in real-time; and (3) the stimulus presentation monitor, which not only presents the stimulus, it can also present the output of the analysis or even raw time course information as a basis for the $\mathrm{BCl}$. A conceptual overview of a possible $\mathrm{BCl}$ setup is shown in Figure 2.

\section{fMRI BCl setup}

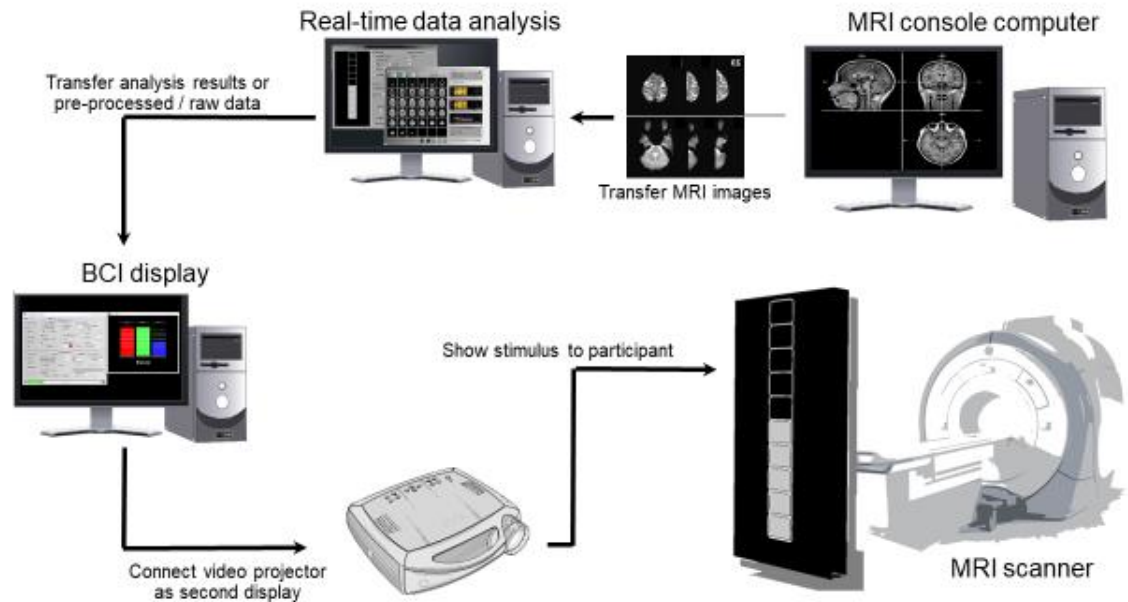

Figure 2: Conceptual rt-fMRI $B C l$ Setup. The fully reconstructed $f M R I$ images are transferred to the real-time analysis computer using a shared network folder or a direct TCP/IP connection. After preprocessing and analysis on the real-time data-analysis machine the data is transferred to the $\mathrm{BCl}$ computer which allows further analysis and presents the stimuli to the participant. Real-time and $\mathrm{BCl}$ computers are separated for best performance and timing precise stimulus presentation. 
Using for example rt-fMRI to decode temporal and spatial properties of the hemodynamics response of the brain allows creating multiple-choice communication $\mathrm{BCl}$ s or even $\mathrm{BCl}$-based letter-spelling devices (Lührs, Sorger, Goebel, \& Esposito, 2017; Sorger et al., 2009; Sorger, Reithler, Dahmen, \& Goebel, 2012) its own brain activity which could lead to improved learning paradigms, more advanced techniques helping to gain more control over their own mind or in noninvasive treatments for certain diseases.

If a participant is also informed about his performance, a communication/control $\mathrm{BCl}$ also includes a neurofeedback component since it projects the (eventually en-/decoded) brain activity back to the participant. This allows the participant to get control over his/her own brain activity which allows for example to create improved learning paradigms (G. Zhang, Yao, Zhang, Long, \& Zhao, 2013), advanced techniques helping to gain more control over their own mind (Christopher DeCharms, 2008) or in noninvasive treatments for certain disease (Scharnowski et al., 2015; J. J. Yoo et al., 2012; Q. Zhang, Zhang, Yao, \& Zhao, 2015). Some example applications to help recovery or symptom improvement of specific illnesses which are targeted till now using rtfMRI imaging techniques range from attention deficit hyperactivity disorder (ADHD) (Alegria et al., 2017; Katya et al., 2016) over binge eating disorder (Sokunbi, 2018) to Stroke recovery (Neyedli et al., 2018; Wang et al., 2017), depression (Linden et al., 2012; Weiskopf, 2012; Young et al., 2017) and more (Thibault et al., 2018). 


\section{IMPROVEMENTS IN REGION OF INTEREST SELECTION}

All these rt-fMRI applications rely on an appropriate delineation of target brain regions. Selecting regions of interests (ROIs) for such experiments can be done using either functional or anatomical atlases or functional localizers (Stoeckel et al., 2014) (e.g., paradigms which allow to contrast specific tasks and activate brain regions of interest). Anatomical atlases can be defined automatically by projecting the fMRI data into the anatomical space (or vice versa) and extract the time course of this area. A potential problem and disadvantage of this procedure is that it relies on the goodness of fit of an individual subject's brain to a common space (e.g., Montreal Neurological Institute (MNI) or Talairach (Jean \& Tournoux, 1988; Talairach, 1967) space). The better the functional to anatomical alignment and the less different the targeted brain areas are, the higher the specificity of the selected region (Greve \& Fischl, 2009). Depending on the size and variance of the targeted area, the anatomical selection can be very precise or rather broad. Using data preprocessing procedures like spatial smoothing can help to improve the appropriateness of the $\mathrm{ROI}$ selection while reducing the spatial resolution of the fMRI data.

A more individualized approach is the so-called functional localizer. For each participant an additional experiment within the scanning session is performed to localize a subject-specific target area. Anatomical information can also be added to verify the location of the target area. This procedure assumes clear knowledge about the specific brain areas which are used for the particular communication/control $\mathrm{BCl}$ or neurofeedback application. Because this implies the availability of experts for each rt-fMRI session, it will be difficult to implement this as a treatment option (Thibault et al., 2018) in a wider spectrum. A possible option to overcome this issue by automatizing the ROI selection is described in the first part of this dissertation. 


\section{IMPROVEMENTS IN TEMPORAL RESOLUTION}

The temporal resolution that can be achieved using standard fMRIsequence parameters with whole-brain coverage usually ranges from 13 s repetition time (TR). This allows to investigate the signal of interest, the blood-oxygen-level dependent (BOLD) contrast (Ogawa, Lee, Kay, \& Tank, 1990). Nevertheless, a lot of information is smeared throughout this time period, e.g., physiological information like cardiac pulsation and respiration as well as motion and other noise artifacts (Posse et al., 2012). More recent methods like single-shot echo-volumar imaging (EVI) (Rabrait et al., 2008), Inverse Imaging (InI) (Lin et al., 2012), highly undersampled projection imaging (PI) (Grotz, Zahneisen, Ella, Zaitsev, \& Hennig, 2009), multiplexed EPI (Feinberg et al., 2010) and last but not least MR-Encephalography (Assländer et al., 2013; Zahneisen et al., 2011, 2012) allow repetition times in the range of $100 \mathrm{~ms}$ or less (Posse et al., 2012). The increased sensitivity resulting from the high sampling rate gives more insights into the BOLD-signal dynamics and allows to at least some degree correct for physiological artifacts which highly contaminate the BOLD-signal. Real-time applications which allow the participants to modulate their own brain activity are therefore especially of interest for this improved sampling rate. It would for example allow ensuring that modulation of the BOLD-response is not caused by adjusting the respiration of the participant which also affects the Bold-Signal (Chang \& Glover, 2009). The potential to remove artifacts (online) is especially relevant/beneficial for real-time applications as the appropriateness of the (e.g., neurofeedback) signal is crucial and has an immediate effect on the ongoing experiment is, for example, not that relevant for standard fMRI experiments for which can optimize the preprocessing at any later point in time (after the data acquisition). So, it is important to investigate the potential benefits specifically gained by MREG also compared to standard $\mathrm{fMRI}$ sequences which use a lower temporal resolution which will be described in chapter three of this thesis. 


\section{TOWARDS MOBILE BCI APPLICATIONS}

One drawback of rt-fMRI is the limited availability and immobility. The costs are rather high, and it is questionable if these will be covered by health insurances soon (or in the upcoming years). A possible alternative to rt-fMRI is the use of rt-fNIRS which allows non-invasive brain spectroscopy of specific target areas. Even though the spatial resolution is only about $3 \mathrm{~cm}$ (Cui, Bray, Bryant, Glover, \& Reiss, 2011), the optode placement can be very precise so that specific areas can be targeted.

Also, the temporal resolution of fNIRS can be within the range of milliseconds which gives the benefits as describe in the previous paragraph. This allows to control also in mobile applications for pulse and respiration artifacts and false modulation strategies.

Since the field of $\mathrm{FMRI}$ and $\mathrm{fNIRS}$ is still quite young compared to methods like EEG, the field of rt-fMRI and rt-fNIRS applications offers many opportunities to support patients in their recovery process. At the same time needs more detailed research in the used methods to sustenance the recovery process in the specific disease. 


\section{RESEARCH AIM AND OUTLINE OF THIS THESIS}

In this thesis, novel tools and methods are presented with the aim to allow researchers and clinicians in the future to apply new $\mathrm{BCl}$ and neurofeedback approaches in different fields. More specifically, the current research applications of rt-fMRI and rt-fNIRS are mainly investigating potential applications (e.g., neurofeedback for specific diseases or $\mathrm{BCls}$ for specific patient groups) but there is a lack of standardized methods to easily use these applications on different sites and environments, for example without the need of researchers, doctors or specifically trained personal to run the experiments.

This implies ensuring that additional methods are developed bridging the gap between research and real-world applications which is the focus of this thesis. Multiple developments towards more systematic applications of current research results will be proposed throughout the following chapters.

First, a method to automatically select ROIs for $\mathrm{BCl}$ is proposed.

\section{Chapter 2 - Automated selection of brain regions for real-time fMRI Brain Computer Interfaces}

This chapter focuses on advancements in rt-fMRI based BCls which use localizer experiments to determine target ROIs as input for the $\mathrm{BCl}$. The ROI selection is usually performed on-site by researchers performing the study. To be able to standardize this procedure and avoid the need of experts to run the $\mathrm{BCl}$ experiment, the automation of this step is a key point. Two unsupervised computational techniques based on the general linear model (GLM) and independent component analysis of rt-fMRI data were developed, and the performances were compared on a communication $\mathrm{BCl}$. 3T $\mathrm{fMRI}$ data of six volunteers were re-analyzed in a simulated real-time condition to evaluate the potential of the two approaches. Participants performed three different mental tasks during a localizer run (e.g., motor imagery, mental calculation, inner speech, or mental singing, all using a visual stimulus). In the two communication runs, a letter-spelling display guided the subjects to freely encode letters by performing one of the mental tasks with a specific timing. The two approaches, GLM- and ICA-based, were used to decode each letter, using 
compact ROls and whole-brain distributed spatio-temporal patterns of fMRI activity respectively, which were automatically defined from subject-specific or group-level maps.

Letter-decoding performances were then compared to supervised methods.

The next chapter covers the advantages of a higher sampling rate in fMRI applications.

\section{Chapter 3 - MR-Encephalography for BCl/Neurofeedback applications with high temporal resolution sampling}

This chapter focuses on the comparison of a standard fMRI sequence using a TR of two seconds with a more recently developed sequence called MR-Encephalography (MREG). Because of the limited temporal resolution, using standard EPI sequences it is not directly possible to separate physiological artifacts such as breathing and heart beat from the fMRI signal which potentially causes misleading false positive effects especially in $\mathrm{BCl}$ and neurofeedback setups. The high temporal resolution of real-time MR-Encephalography (rt-MREG) allows to un-aliase and correct for physiological fluctuations and leads to increased sensitivity for mapping task-based activation and functional connectivity as well as for detecting dynamic changes in connectivity over time (Feinberg et al., 2010; Posse et al., 2013). The larger number of data points also holds the potential for faster detection of resting-state (Akin, Lee, Hennig, \& LeVan, 2017). Moreover, BOLD-signal changes have been shown to contain measurable fluctuations at frequencies up to $5 \mathrm{~Hz}$, which are not accessible by conventional whole-brain echo planar imaging (EPI) data. MREG allows 3D whole-brain imaging with sampling rates below $100 \mathrm{~ms}$ per volume and a nominal spatial resolution of 3-mm isotropic using a spherical read-out on a $3 \mathrm{~T} \mathrm{MRI}$. In this chapter, we present results obtained by offline analyses and discuss the potential of this technique for future rt-fMRI applications.

In the last chapter, a newly developed software for analyzing fNIRS data in real-time and enabling communication/control $\mathrm{BCls}$ and neurofeedback applications is introduced. Since the fNIRS research field 
is still lacking a user-friendly and powerful real-time data-analysis software this is especially important for a continuous progress in the field.

\section{Chapter 4 - Turbo-Satori - A neurofeedback and BCl toolbox for real- time fNIRS}

This chapter focuses on a neurofeedback and $\mathrm{BCl}$ toolbox for real-time fNIRS called Turbo-Satori. While the first two chapters focus more on improving methods for real-time applications, this chapter changes gears towards a wider spread range of applications also using hemodynamic signals. Since fNIRS is also a mobile system the possible applications of this techniques are much broader compared to fMRI which was the bases of chapters two and three. Hereby the developed toolbox should also provide improvements in $\mathrm{BCl}$ applications. One important aspect thereby is the possibility of a fast and reliable experiment setup and the execution in a real-time environment. Also, statistical analysis methods, suitable for real-time, should be part of such a toolbox. Including incremental recursive least-squares procedure for real-time general linear model (rlsGLM) and support vector machine (SVM) classifiers for advanced BCl applications. Flexible input and output routines for real-time applications that allow to interface with other $3^{\text {rd }}$-party stimulus applications to access the processed data and calculated statistics in real-time was also seen as a very important component of the toolbox. Overall the toolbox should allow an immediate access to real-time fNIRS data analysis and $\mathrm{BCl}$ paradigms. 


\section{REFERENCES}

Akin, B., Lee, H. L., Hennig, J., \& LeVan, P. (2017). Enhanced subject-specific resting-state network detection and extraction with fast $\mathrm{fMRI}$. Human Brain Mapping, 38(2), 817-830. https://doi.org/10.1002/hbm.23420

Alegria, A. A., Wulff, M., Brinson, H., Barker, G. J., Norman, L. J., Brandeis, D., ... Rubia, K. (2017). Real-time fMRI neurofeedback in adolescents with attention deficit hyperactivity disorder. Human Brain Mapping, 38(6), 3190-3209. https://doi.org/10.1002/hbm.23584

Assländer, J., Zahneisen, B., Hugger, T., Reisert, M., Lee, H. L., LeVan, P., \& Hennig, J. (2013). Single shot whole brain imaging using spherical stack of spirals trajectories. Neurolmage, 73, 59-70. https://doi.org/10.1016/j.neuroimage.2013.01.065

Bagarinao, E., Nakai, T., \& Tanaka, Y. (2006). Real-time functional MRI: development and emerging applications. Magn Reson Med Sci, 5(3), 157165. https://doi.org/10.2463/mrms.5.157

Birbaumer, N., Murguialday, A. R., \& Cohen, L. (2008). Brain-computer interface in paralysis. Current Opinion in Neurology, 21(6), 634-638. https://doi.org/10.1097/WCO.0b013e328315ee2d

Boas, D. A., Elwell, C. E., Ferrari, M., \& Taga, G. (2014). Twenty years of functional near-infrared spectroscopy: Introduction for the special issue. Neurolmage, 85, 1-5. https://doi.org/10.1016/j.neuroimage.2013.11.033

Calderon-Arnulphi, M., Alaraj, A., \& Slavin, K. V. (2009). Near infrared technology in neuroscience: past, present and future. Neurological Research, 31(6), 605-614. https://doi.org/10.1179/174313209X383286

Chang, C., \& Glover, G. H. (2009). Relationship between respiration, end-tidal $\mathrm{CO} 2$, and BOLD signals in resting-state fMRI. Neurolmage, 47(4), 13811393. https://doi.org/10.1016/J.NEUROIMAGE.2009.04.048

Christopher DeCharms, R. (2008, September 1). Applications of real-time fMRI. Nature Reviews Neuroscience. Nature Publishing Group. https://doi.org/10.1038/nrn2414

Cui, X., Bray, S., Bryant, D. M., Glover, G. H., \& Reiss, A. L. (2011). A quantitative comparison of NIRS and fMRI across multiple cognitive tasks. Neurolmage, 54(4), 2808-2821. https://doi.org/10.1016/j.neuroimage.2010.10.069

DeCharms, R. C., Christoff, K., Glover, G. H., Pauly, J. M., Whitfield, S., \& Gabrieli, J. D. E. (2004). Learned regulation of spatially localized brain activation using real-time fMRI. Neurolmage, 21(1), 436-443. https://doi.org/10.1016/j.neuroimage.2003.08.041

Esposito, F., Seifritz, E., Formisano, E., Morrone, R., Scarabino, T., Tedeschi, G., ... Di Salle, F. (2003). Real-time independent component analysis of fMRI time-series.

Neurolmage, 20(4), 2209-2224. 
https://doi.org/10.1016/j.neuroimage.2003.08.012

Feinberg, D. A., Moeller, S., Smith, S. M., Auerbach, E., Ramanna, S., Glasser, M. F., ... Yacoub, E. (2010). Multiplexed echo planar imaging for sub-second whole brain fmri and fast diffusion imaging. PLOS ONE, 5(12), e15710. https://doi.org/10.1371/journal.pone.0015710

Feinberg, D. A., \& Yacoub, E. (2012). The rapid development of high speed, resolution and precision in fMRI. Neurolmage, 62(2), 720-725. https://doi.org/10.1016/j.neuroimage.2012.01.049

Ferrari, M., Giannini, I., Sideri, G., \& Zanette, E. (1985). Continuous Non Invasive Monitoring of Human Brain by Near Infrared Spectroscopy (pp. 873-882). Springer, Boston, MA. https://doi.org/10.1007/978-1-4684-3291-6_88

Greve, D. N., \& Fischl, B. (2009). Accurate and robust brain image alignment using boundary-based registration. Neurolmage, 48(1), 63-72. https://doi.org/10.1016/j.neuroimage.2009.06.060

Grotz, T., Zahneisen, B., Ella, A., Zaitsev, M., \& Hennig, J. (2009). Fast functional brain imaging using constrained reconstruction based on regularization using arbitrary projections. Magnetic Resonance in Medicine, 62(2), 394405. https://doi.org/10.1002/mrm.22009

Jean, T., \& Tournoux, P. (1988). Co-Planar Stereotaxic Atlas of the Human Brain: 3-D Proportional System: An Approach to Cerebral Imaging (Thieme Classics): J. Talairach: 9780865772939: Amazon.com: Books. Thieme. G. Thieme. Retrieved from http://www.amazon.com/Co-Planar-StereotaxicAtlas-Human-Brain/dp/0865772932

Jöbsis, F. F. (1977). Noninvasive, infrared monitoring of cerebral and myocardial oxygen sufficiency and circulatory parameters. Science (New York, N.Y.), 198(4323), 1264-1267. https://doi.org/10.1126/SCIENCE.929199

Katya, R., Analucia, A., Melanie, W., Helen, B., Vincent, G., Gareth, B., ... Anthony, D. (2016). A randomized controlled clinical trial of real-time functional magnetic resonance imaging neurofeedback for adolescents with attention deficit hyperactivity disorder (ADHD). Frontiers in Human Neuroscience, 10. https://doi.org/10.3389/conf.fnhum.2016.220.00060

Kidd, D., Barkhof, F., McConnell, R., Algra, P. R., Allen, I. V, \& Revesz, T. (1999). Home - PubMed - NCBI. Retrieved May 8, 2018, from http://www.ncbi.nlm.nih.gov/pubmed/

La Vaque, T. J. (1999). The History of EEG Hans Berger. Journal of Neurotherapy, 3(2), 1-9. https://doi.org/10.1300/J184v03n02_01

Lin, F. H., Tsai, K. W. K., Chu, Y. H., Witzel, T., Nummenmaa, A., Raij, T., ... Belliveau, J. W. (2012). Ultrafast inverse imaging techniques for fMRI. Neurolmage, 62(2), 699-705.

https://doi.org/10.1016/j.neuroimage.2012.01.072

Linden, D. E. J., Habes, I., Johnston, S. J., Linden, S., Tatineni, R., Subramanian, L., ... Goebel, R. (2012). Real-time self-regulation of emotion networks in 
patients with depression. PLoS ONE, 7(6), e38115. https://doi.org/10.1371/journal.pone.0038115

Lloyd-Fox, S., Blasi, A., \& Elwell, C. E. (2010). Illuminating the developing brain: The past, present and future of functional near infrared spectroscopy. Neuroscience and Biobehavioral Reviews, 34(3), 269-284. https://doi.org/10.1016/j.neubiorev.2009.07.008

Logothetis, N. K., \& Pfeuffer, J. (2004). On the nature of the BOLD fMRI contrast mechanism. Magnetic Resonance Imaging, 22(10), 1517-1531. https://doi.org/10.1016/j.mri.2004.10.018

Lührs, M., Sorger, B., Goebel, R., \& Esposito, F. (2017). Automated selection of brain regions for real-time $\mathrm{fMRI}$ brain-computer interfaces. Journal of Neural Engineering, 14(1), 016004. https://doi.org/10.1088/17412560/14/1/016004

Naseer, N., \& Hong, K.-S. (2015). fNIRS-based brain-computer interfaces: a review. Frontiers in Human Neuroscience, 9(JAN), 3. https://doi.org/10.3389/fnhum.2015.00003

Neyedli, H. F., Sampaio-Baptista, C., Kirkman, M. A., Havard, D., Lührs, M., Ramsden, K., ... Johansen-Berg, H. (2018). Increasing Lateralized Motor Activity in Younger and Older Adults using Real-time fMRI during Executed Movements.

Neuroscience, 378 ,

165-174. https://doi.org/10.1016/j.neuroscience.2017.02.010

Ogawa, S., Lee, T. -M, Nayak, A. S., \& Glynn, P. (1990). Oxygenation-sensitive contrast in magnetic resonance image of rodent brain at high magnetic fields. Magnetic Resonance in Medicine, 14(1), 68-78. https://doi.org/10.1002/mrm.1910140108

Ogawa, S., Lee, T. M., Kay, A. R., \& Tank, D. W. (1990). Brain magnetic resonance imaging with contrast dependent on blood oxygenation. Proceedings of the National Academy of Sciences, 87(24), 9868-9872. https://doi.org/10.1073/pnas.87.24.9868

Posse, S., Ackley, E., Mutihac, R., Rick, J., Shane, M., Murray-Krezan, C., ... Speck, O. (2012). Enhancement of temporal resolution and BOLD sensitivity in real-time fMRI using multi-slab echo-volumar imaging. Neurolmage, 61(1), 115-130. https://doi.org/10.1016/j.neuroimage.2012.02.059

Posse, S., Binkofski, F., Schneider, F., Gembris, D., Frings, W., Habel, U., ... Eickermann, T. (2000). A new approach to measure single-event related brain activity using real-time fMRI: Feasibility of sensory, motor, and higher cognitive tasks. Human Brain Mapping, 12(1), 25-41. https://doi.org/10.1002/1097-0193(200101)12:1<25::AIDHBM30>3.0.CO;2-H

Posse, S., Fitzgerald, D., Gao, K., Habel, U., Rosenberg, D., Moore, G. J., \& Schneider, F. (2003). Real-time fMRI of temporolimbic regions detects amygdala activation during single-trial self-induced sadness. Neurolmage, 
18(3), 760-768. https://doi.org/10.1016/S1053-8119(03)00004-1

Rabrait, C., Ciuciu, P., Ribés, A., Poupon, C., Le Roux, P., Dehaine-Lambertz, G., ... Lethimonnier, F. (2008). High temporal resolution functional MRI using parallel echo volumar imaging. Journal of Magnetic Resonance Imaging, 27(4), 744-753. https://doi.org/10.1002/jmri.21329

Scharnowski, F., Veit, R., Zopf, R., Studer, P., Bock, S., Diedrichsen, J., ... Weiskopf, N. (2015). Manipulating motor performance and memory through real-time fMRI neurofeedback. Biological Psychology, 108, 85-97. https://doi.org/10.1016/j.biopsycho.2015.03.009

Schlereth, F. H., Fossaceca, J. M., Keckler, A. D., \& Barbour, R. L. (n.d.). Multicomputer-based neural networks for imaging in random media. In Conference Record of the 1991 IEEE Nuclear Science Symposium and Medical Imaging Conference (pp. 2193-2197). IEEE. https://doi.org/10.1109/NSSMIC.1991.259308

Sokunbi, M. O. (2018). Using real-time fMRI brain-computer interfacing to treat eating disorders. Journal of the Neurological Sciences, 388, 109-114. https://doi.org/10.1016/j.jns.2018.03.011

Sorger, B., Dahmen, B., Reithler, J., Gosseries, O., Maudoux, A., Laureys, S., \& Goebel, R. (2009). Another kind of "BOLD Response": answering multiplechoice questions via online decoded single-trial brain signals. Progress in Brain Research, 177(C), 275-292. https://doi.org/10.1016/S00796123(09)17719-1

Sorger, B., Reithler, J., Dahmen, B., \& Goebel, R. (2012). A real-time fMRI-based spelling device immediately enabling robust motor-independent communication. Current Biology, 22(14), 1333-1338. https://doi.org/10.1016/j.cub.2012.05.022

Stoeckel, L. E., Garrison, K. A., Ghosh, S., Wighton, P., Hanlon, C. A., Gilman, J. M., ... Evins, A. E. (2014). Optimizing real time fMRI neurofeedback for therapeutic discovery and development. Neurolmage: Clinical, 5, 245-255. https://doi.org/10.1016/j.nicl.2014.07.002

Sulzer, J., Haller, S., Scharnowski, F., Weiskopf, N., Birbaumer, N., Blefari, M. L., ... Sitaram, R. (2013). Real-time fMRI neurofeedback: Progress and challenges. Neurolmage, 76, 386-399. https://doi.org/10.1016/j.neuroimage.2013.03.033

Talairach, J. (1967). Atlas d'anatomie stéréotaxique du télencéphale : études anatomo-radiologiques. Paris: Masson. Retrieved from http://www.worldcat.org/title/atlas-danatomie-stereotaxique-dutelencephale-etudes-anatomo-radiologiques/oclc/249360510

Thibault, R. T., MacPherson, A., Lifshitz, M., Roth, R. R., \& Raz, A. Neurofeedback with fMRI: A critical systematic review, 172 Neurolmage $\S$ (2018). Academic Press. https://doi.org/10.1016/j.neuroimage.2017.12.071

Torricelli, A., Contini, D., Pifferi, A., Caffini, M., Re, R., Zucchelli, L., \& Spinelli, L. 
(2014). Time domain functional NIRS imaging for human brain mapping. Neurolmage, 85 ,

28-50. https://doi.org/10.1016/j.neuroimage.2013.05.106

Voyvodic, J. T. (1999). Real-time fMRI paradigm control, physiology, and behavior combined with near real-time statistical analysis. Neurolmage, 10(2), 91-106. https://doi.org/10.1006/nimg.1999.0457

Wald, L. L. (2012). The future of acquisition speed, coverage, sensitivity, and resolution. Neurolmage, $62(2)$,

1221-1229. https://doi.org/10.1016/j.neuroimage.2012.02.077

Wang, T., Mantini, D., \& Gillebert, C. R. (2017). The potential of real-time fMRI neurofeedback for stroke rehabilitation: A systematic review. Cortex. https://doi.org/10.1016/j.cortex.2017.09.006

Weiskopf, N. (2012). Real-time fMRI and its application to neurofeedback. Neurolmage, 62(2), 682-692. https://doi.org/10.1016/j.neuroimage.2011.10.009

Yoo, J. J., Hinds, O., Ofen, N., Thompson, T. W., Whitfield-Gabrieli, S., Triantafyllou, C., \& Gabrieli, J. D. E. (2012). When the brain is prepared to learn: Enhancing human learning using real-time fMRI. Neurolmage, 59(1), 846-852. https://doi.org/10.1016/j.neuroimage.2011.07.063

Yoo, S.-S., Fairneny, T., Chen, N.-K., Choo, S.-E., Panych, L. P., Park, H., ... Jolesz, F. A. (2004). Brain-computer interface using fMRI: spatial navigation by thoughts. NeuroReport, 15(10), 1591-1595. https://doi.org/10.1097/01.wnr.0000133296.39160.fe

Yoo, S.-S., \& Jolesz, F. A. (2002). Functional MRI for neurofeedback: feasibility studyon a hand motor task. Neuroreport, 13(11), 1377-1381. https://doi.org/10.1097/00001756-200208070-00005

Young, K. D., Siegle, G. J., Zotev, V., Phillips, R., Misaki, M., Yuan, H., ... Bodurka, J. (2017). Randomized clinical trial of real-time fMRI amygdala neurofeedbackfor major depressive disorder: Effectson symptoms and autobiographical memory recall. American Journal of Psychiatry, 174(8), 748-755. https://doi.org/10.1176/appi.ajp.2017.16060637

Zahneisen, B., Grotz, T., Lee, K. J., Ohlendorf, S., Reisert, M., Zaitsev, M., \& Hennig, J. (2011). Three-dimensional MR-encephalography: Fast volumetric brain imaging using rosette trajectories. Magnetic Resonance in Medicine, 65(5), 1260-1268. https://doi.org/10.1002/mrm.22711

Zahneisen, B., Hugger, T., Lee, K. J., Levan, P., Reisert, M., Lee, H. L., ... Hennig, J. (2012). Single shot concentric shells trajectories for ultra fast fMRI. Magnetic Resonance in Medicine, 68(2), 484-494. https://doi.org/10.1002/mrm.23256

Zhang, G., Yao, L., Zhang, H., Long, Z., \& Zhao, X. (2013). Improved Working Memory Performance through Self-Regulation of Dorsal Lateral Prefrontal Cortex Activation Using Real-Time fMRI. PLOS ONE, 8(8), e73735. 
https://doi.org/10.1371/journal.pone.0073735

Zhang, Q., Zhang, G., Yao, L., \& Zhao, X. (2015). Impact of real-time fMRI working memory feedback training on the interactions between three core brain networks. Frontiers in Behavioral Neuroscience, 9, 244. https://doi.org/10.3389/fnbeh.2015.00244 


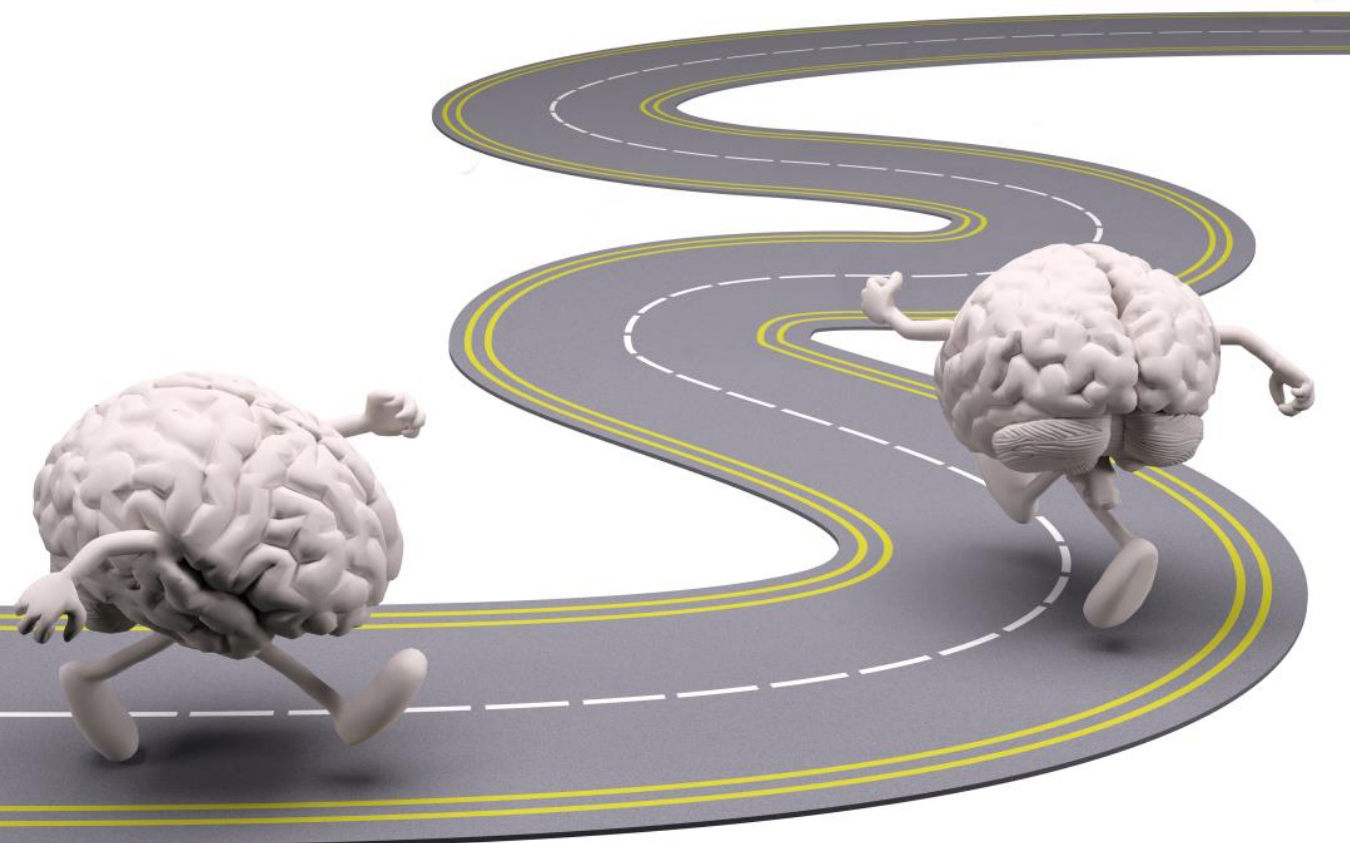




\section{Chapter 2}

\section{Automated selection of brain regions for real-time fMRI Brain Computer Interfaces}

Corresponding publication:

Lührs, M., Sorger, B., Goebel, R., Esposito, F., 2016. Automated selection of brain regions for real-time fMRI brain-computer interfaces. Journal of Neural Engineering, 14(1), 16004. 


\section{ABSTRACT}

Objective. Brain-computer interfaces ( $\mathrm{BCls})$ implemented with real-time functional magnetic resonance imaging (rt-fMRI) use fMRI time-courses from predefined regions of interest (ROIs). To reach best performances, localizer experiments and on-site expert supervision are required for ROI definition. To automate this step, we developed two unsupervised computational techniques based on the general linear model (GLM) and independent component analysis (ICA) of rt-fMRI data, and compared their performances on a communication $\mathrm{BCl}$.

Approach. 3 Tesla fMRI data of six volunteers were re-analyzed in simulated real-time. During a localizer run, participants performed three mental tasks following visual cues. During two communication runs, a letter-spelling display guided the subjects to freely encode letters by performing one of the mental tasks with a specific timing. GLM- and ICAbased procedures were used to decode each letter, respectively using compact ROls and whole-brain distributed spatio-temporal patterns of fMRI activity, automatically defined from subject-specific or group-level maps.

Main Results. Letter-decoding performances were comparable to supervised methods. In combination with a similarity-based criterion, GLM- and ICA-based approaches successfully decoded more than $80 \%$ (average) of the letters. Subject-specific maps yielded optimal performances.

Significance. Automated solutions for ROI selection may help accelerating the translation of $\mathrm{rt}-\mathrm{fMRI} \mathrm{BCls}$ from research to clinical applications. 


\section{INTRODUCTION}

Real-time functional magnetic resonance imaging (rt-fMRI) allows immediate access to brain activity information by analyzing blood oxygen level dependent (BOLD) activation signals as fast as they are acquired (Weiskopf, 2012). For this reason, rt-fMRI can be used as a brain-computer interface $(\mathrm{BCl})$ with high spatial resolution and wholebrain coverage, thereby overcoming some of the limitations of electroencephalography (EEG) based BCls (Weiskopf, 2012). Rt-fMRI $\mathrm{BCls}$ can also be used for neurofeedback (Ruiz, Buyukturkoglu, Rana, Birbaumer, \& Sitaram, 2014; Stoeckel et al., 2014; Sulzer et al., 2013; Weiskopf, 2012). In these applications, the rt-fMRI BCl is expressly designed to implement the real-time feedback of BOLD signals to the experimental subject or the patient. Thereby, researchers and clinicians can use rt-fMRI BCls to train individual subjects to self-regulate their brain activity in various different brain areas, and possibly observe specific behavioral or therapeutic effects across repeated sessions of the experiment (Ruiz et al., 2014; Stoeckel et al., 2014; Sulzer et al., 2013; Weiskopf, 2012).

Although $f M R I$ is more costly than EEG, rt-fMRI $B C l$ s may offer unique opportunities, especially in the field of communication and control BCls (Lorina Naci et al., 2012). For instance, in recent studies, it has been shown that participants were able to control brain activation within different brain regions distinctively, e.g., to answer multiple-choice questions or to navigate through a virtual maze (Eklund, Andersson, Ohlsson, Ynnerman, \& Knutsson, 2010; Sorger et al., 2009; Yoo et al., 2004). A more recent study (Sorger, Reithler, Dahmen, \& Goebel, 2012) introduced a motor-independent communication technique that allowed decoding up to 27 distinct information units. The communication $\mathrm{BCl}$ introduced by Sorger and colleagues (Sorger et al., 2012) enables bi-directional communication using BOLD signals in predefined brain regions of interest (ROIs). In fact, using this letterspelling rt-fMRI $\mathrm{BCl}$, participants were able to communicate freely chosen answers to open questions.

From a technical standpoint, both rt-fMRI communication/control and neurofeedback $\mathrm{BCl}$ s rely on a general $\mathrm{rt}-\mathrm{fMRI}$ analysis pipeline, thereby similar issues pertain to, and common solutions are shared between, the two applications. Among these, the selection of the ROls, as either 
$\mathrm{BCl}$ signal source(s) or neurofeedback target location(s), is considered critical for both $\mathrm{BCl}$ decoding performances and for neurofeedback treatment success (Ruiz et al., 2014; Sokunbi, Linden, Habes, Johnston, \& Ihssen, 2014). In previous rt-fMRI works, depending on the specific application, source/target ROls have been typically obtained using (i) available anatomical atlases (see, e. g., (Koush et al., 2015)), (ii) thresholded group-level functional maps from separate fMRI experiments (see, e. g., (Lorenz et al., 2016)) or (iii) subject-specific functional maps from within-session localizer experiments (see, e. g., (Cohen Kadosh et al., 2016)).

In order to reach best possible decoding and neurofeedback performances, the selection of ROls in fMRI-based $\mathrm{BCls}$ is usually optimized within the specific application. This can be achieved with carefully designed functional localizer experiments prior to the actual $\mathrm{BCl}$ experiment. However, in this case, multi-domain cognitive expertise (especially about functional network topographies) as well as on-line supervision capabilities to manually set appropriate statistical models and thresholds are crucial to optimally select the relevant task-related brain regions from localizer data sets. These aspects may impede the use of $\mathrm{fMRI}$-based $\mathrm{BCls}$ in clinical setups due to the need of human experts. In addition, because fMRI responses and patterns may change significantly in patients, due to, e.g., unknown levels of cognitive capacities, attention, arousal and communication intents (see, e.g., (L Naci, Cusack, Jia, \& Owen, 2013)), the same spatial and temporal models used for a given setup in healthy volunteers, might not work with identical performances in clinical settings.

In an attempt to automatize the procedure of selecting $\mathrm{fMRI}$-based $\mathrm{BCl}$ signal sources, we developed two novel unsupervised computational approaches, one based on the standard general linear model (GLM) (Nelder \& Wedderburn, 1972) and one based on real-time independent component analysis (ICA) (Esposito et al., 2003), and (re-)analyze data presented in (Sorger et al., 2012) and an additional data set, in a simulated real-time framework. Although the proposed methods were applied in off-line data analyses of existing data, which could in part limit the general validity of the presented results, we present a novel and fully automatic processing pipeline that can be used as is in an actual real-time setup. 
We hypothesized that using the proposed selection strategies and algorithms, comparable performances between the proposed unsupervised solutions and the previous supervised solutions could be achieved. Because our initial simulations clearly indicated that the implemented pipeline is fully compatible with real-time applications, the results obtained with the presented approaches can be useful for the implementation of a fully automated rt-fMRI BCI. More in general, these approaches could be applied in other communication/control and neurofeedback $\mathrm{BCls}$, e.g., to enhance effective connectivity (Koush et al., 2015), to develop new experimental paradigms in cognitive neuroscience (Lorenz et al., 2016) or for closed-loop training (Megan, Cohen, Lee, Norman, \& Turk-browne, 2015). 


\section{METHODS}

\section{MRI Data Description of the fMRI Data Sets}

(F)MRI data of six participants (mean age $29.3+$ +/ 5.39 years, four female and two male) were re-analyzed. Details about the population can be found in table 1, including participants' age, sex, handedness (as evaluated by the Edinburgh Inventory (Oldfield, 1971)), and prior fMRI experience. Moreover, it is indicated how many volumes were acquired in each functional run (Sorger et al., 2012). For subject S1 data of two sessions were available, of which the second data set was not included in the previous study (Sorger et al., 2012). 


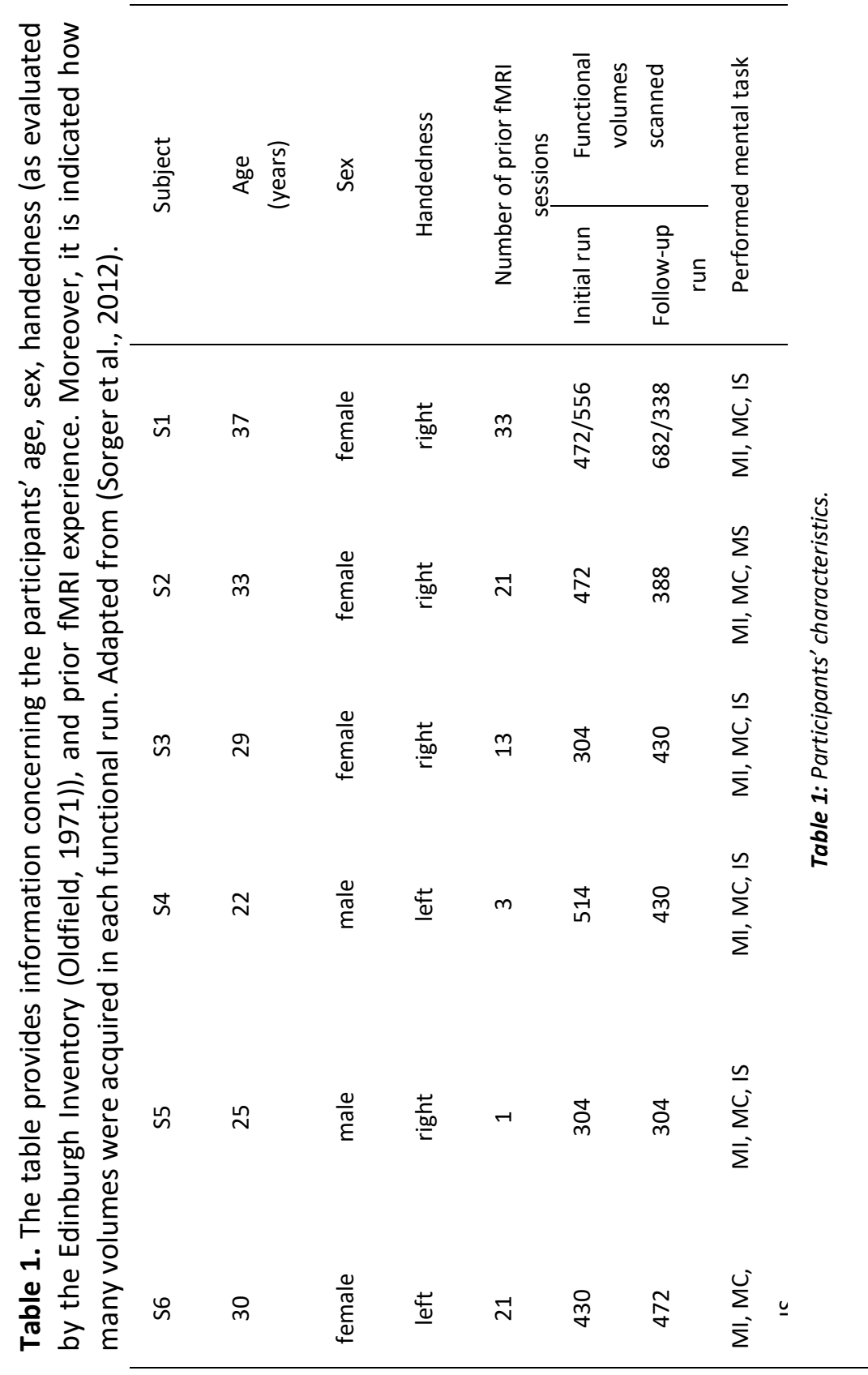

Remarks: $M I=$ motor imagery, $M C=$ mental calculation, $I S=$ inner speech, MS = mental singing. 
Description of the $\mathrm{BCl}$ and Experimental Design

The background of the letter encoding technique and all details about the experimental design can also be found in (Sorger et al., 2012). For the sake of comprehension of the present paper, the description of the $\mathrm{BCl}$ has been repeated here.

In order to represent each letter of the English alphabet and the blank space by a distinct neural activation pattern, a letter encoding technique was implemented that requires almost zero pretraining. This technique exploits spatiotemporal characteristics of single-trial BOLD signals that are associated with the participant's performance of different mental tasks and certain temporal aspects of task execution. That a single cognitive event can be assessed online has been demonstrated by Posse and colleagues (Posse et al., 2000). To intentionally generate 27 distinguishable hemodynamic activation patterns, participants voluntarily influenced three BOLD signal aspects: (i) location of the signal source by performing three different mental tasks, (ii) signal onset delay by delaying the start of the mental task for $0 \mathrm{~s}, 10 \mathrm{~s}$, or $20 \mathrm{~s}$, and (iii) signal duration by varying the mental task duration between $10 \mathrm{~s}, 20$ $\mathrm{s}$, and $30 \mathrm{~s}$. Full combination of these parameters results in the generation of 27 unique hemodynamic brain responses, which can be assigned to 27 characters (figure 1). The letter-by-letter translation of any freely chosen answer into single-trial BOLD signals required only little effort from participants as the encoding process was facilitated by using a convenient visual letter encoding display designed according to the coding scheme (figure 1). For encoding a certain letter, participants merely had to attend to the desired letter and to perform the designated mental task as long as the letter was highlighted in the display (figure 2). 


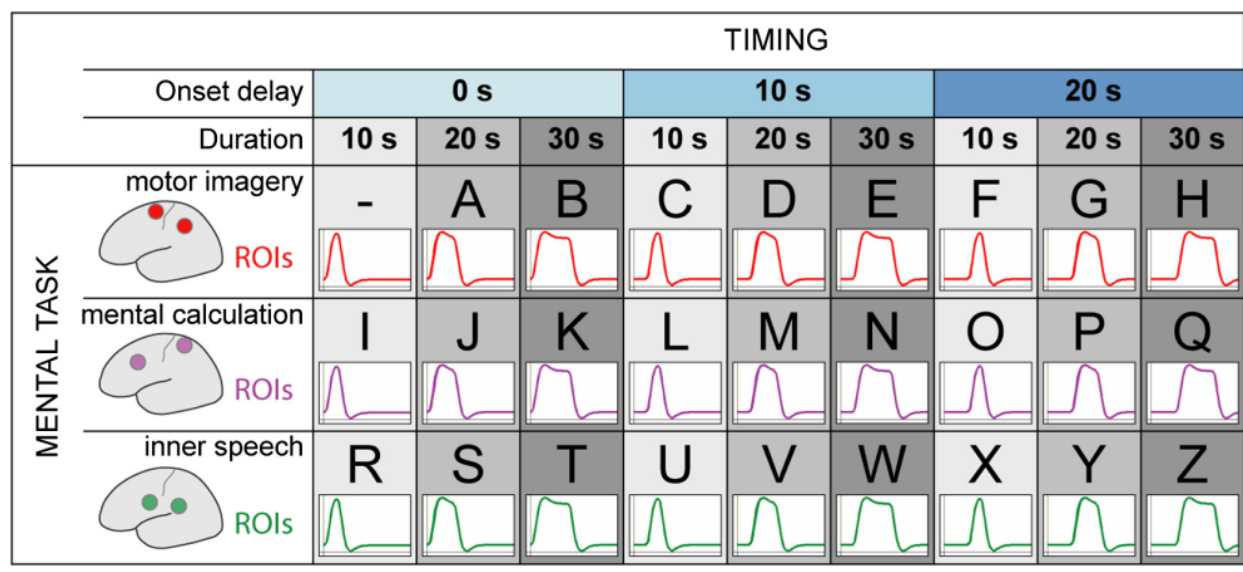

Figure 1: Letter Coding Scheme. Combining three mental tasks, three task onset delays, and three task durations allows for encoding $3 \times 3 \times 3$ $=27$ distinct information units. When assigning these 27 combinations to 26 letters and the blank space ("-"), every character can be represented by a certain cognitive event leading to a unique dynamic brain response pattern. In order to encode, e.g., the letter " $E$," a motor action has to be imagined, starting $10 \mathrm{~s}$ after the general trial onset and lasting for $30 \mathrm{~s}$. Remarks/abbreviations are as follows: RTCS, reference time courses; ROIs, regions of interest; see Table S1 for mental tasks and ROIs used in the current study; see (Sorger et al., 2012) Movie S1 for brain activation patterns evoked by performing different mental tasks; curves shown are modeled RTCS derived from standard hemodynamic response functions (note, however, that individual RTCs were used for letter decoding [see (Sorger et al., 2012) figure S3 for comparison of individual versus standard RTCs]). Figure reused from (Sorger et al., 2012) with permission by the publisher.

After each letter encoding trial, the participants had to passively view the display and rest until the next trial. During this trial, no feedback was given to the participants. According to previous (EEG-based) $\mathrm{BCl}$ studies (see, e. g., (Lorenz, Pascual, Blankertz, \& Vidaurre, 2014; Reuderink, Nijholt, \& Poel, 2009)), both motivation and frustration can be increased by feedback and error monitoring could lead to changes in $\mathrm{BCl}$ performances. In this case, it was decided to not give letter by letter feedback to prevent frustration, also considering that not every letter needs to be correct in order to later be able to successfully interpret the whole word. 
In a localizer experiment preceding the experimental runs, the participants were instructed to perform one of the three different tasks as soon and for as long as the word of the task (e.g., motor imagery) was shown on the display. To later derive individual response functions for each participant, the three different tasks where shown for three different time intervals (10, 20 and 30 seconds).

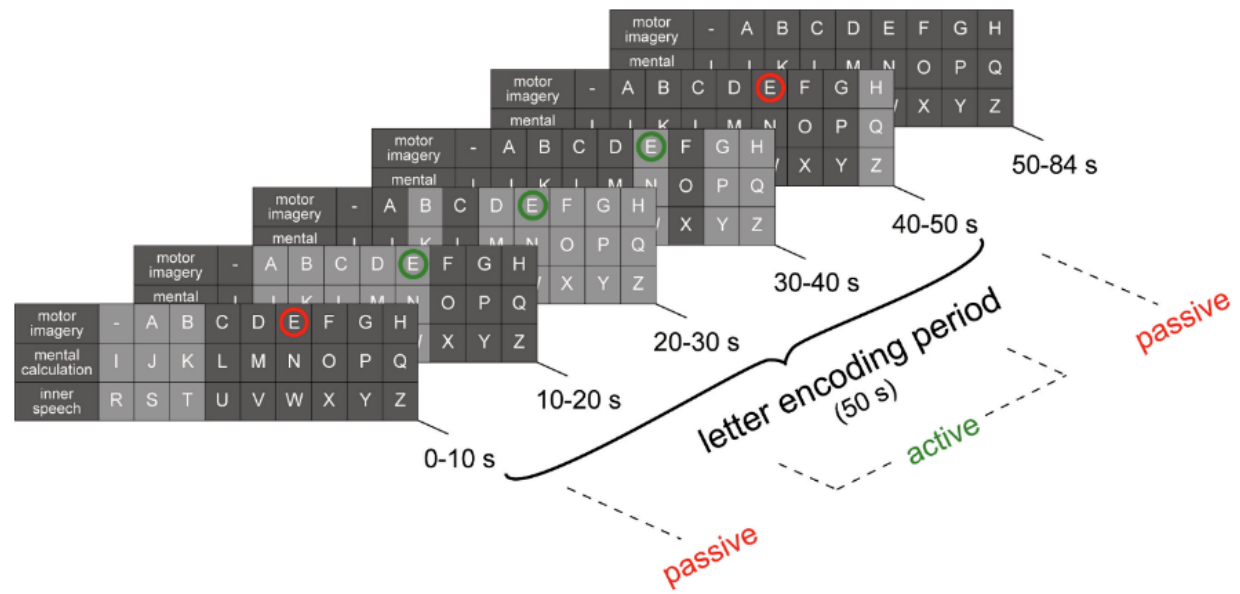

Figure 2: In order to encode a specific letter, participants did not have to memorize the encoding parameters for a certain letter (i.e., mental task, onset delay, task duration). They only had to attend to the selected letter and to perform the particular mental task assigned to the respective row (indicated in the first column of each window) as long as the accordant letter cell was highlighted (for $10 \mathrm{~s}, 20 \mathrm{~s}$, or $30 \mathrm{~s}$ ). In order to encode, e.g., the letter " $E$," no mental task has to be performed in the first $10 \mathrm{~s}$ following the onset of the letter encoding trial (passive). When the " $E$ " cell gets highlighted, the participant starts performing "motor imagery" (active). As soon as the letter cell is no longer highlighted (after $30 \mathrm{~s}$ in this example), the participant stops and stays focusing on " $E$ " for another $10 \mathrm{~s}$ (passive) until the whole letter encoding period is finished. During the subsequent resting period, in which none of the letter cells is highlighted, the participant is asked to select (and switch to) the next intended letter remaining in a sort of "stand-by" mode, selecting the next mental task to be performed, and awaiting the next active letter encoding phase. Note that the conceptualization of our letter encoding display and the visual display invented in the context of the development of an EEG-based spelling device via event-related potentials (P300) (Farwell \& Donchin, 1988) share common characteristics. Remarks are as 
follows: circles were not visible for participants; they are shown here in order to emphasize active (green) and passive (red) answer encoding phases when encoding the letter " $E$ ". Figure reused from (Sorger et al., 2012) with permission by the publisher.

\section{MRI Data Acquisition}

FMRI data were acquired with a 3-T MRI head scanner (Magnetom Allegra, Siemens Healthcare, Erlangen, Germany) using a quadrature birdcage head coil. In all sessions, a single-shot repeated echo-planar imaging (EPI) sequence was used to produce BOLD-fMRI image time series. Except for the number of acquisitions (localizer experiment: 372 volumes; for further details, see Table 1), identical scanning parameters were used for both experimental runs (repetition time [TR] = $2000 \mathrm{ms,}$ echo time $[\mathrm{TE}]=30 \mathrm{~ms}$, flip angle $[\mathrm{FA}]=90^{\circ}$, field of view $[\mathrm{FOV}]=224 \times$ $224 \mathrm{~mm} 2$, matrix size $=64 \times 64$, number of slices $=34$, slice thickness $=$ $3.5 \mathrm{~mm}$, no gap, slice order = ascending/interleaved).

For each participant, a three-dimensional (3D) T1-weighted data set encompassing the whole brain was acquired following the localizer experiment (scan parameters: $\mathrm{TR}=2250 \mathrm{~ms}$, TE $=2.6 \mathrm{~ms}, \mathrm{FA}=9$ ㅇ, $\mathrm{FOV}=$ $256 \times 256 \mathrm{~mm}^{2}$, matrix size $=256 \times 256$, number of slices $=192$, slice thickness $=1 \mathrm{~mm}$, total scan time $=8 \mathrm{~min}$ and $26 \mathrm{~s}$ ). Parameters of this anatomical MRI sequence were based on the Alzheimer's disease Neuroimaging Initiative protocols. Anatomical and functional MRI offline data processing was performed using BrainVoyager QX (v2.8.4; Brain Innovation B.V., Maastricht, The Netherlands). Further details about the MRI data acquisition protocol can be found in (Sorger et al., 2012).

\section{MRI Data Analysis}

Image data preprocessing (motion correction, spatial smoothing with gaussian kernel FWHM $6 \mathrm{~mm}$ ) and simulated real-time analyses were performed using Turbo-BrainVoyager (v3.2; Brain Innovation B.V., Maastricht, The Netherlands). No trend removal (high-pass filtering) was applied on the motion-corrected EPI time series to simulate the realtime analysis framework but added as a confound predictor to the GLM analysis.

The automated decoding procedures (see details below) for the ICAbased algorithm (section 2.6) was implemented in Matlab (The 
MathWorks, Inc., Natick, Massachusetts, USA) using the output of Turbo-BrainVoyager and the rt-ICA plugin of Turbo-BrainVoyager, that respectively consisted of the $\mathrm{ROI}$ time-courses and the ICA component maps and representative time-courses.

Image data spatial normalization and template ROI definitions were performed using BrainVoyager QX (v2.8.4 Brain Innovation B.V., Maastricht, The Netherlands).

\section{GLM-based Letter Decoding}

The fMRI data of the functional localizer run were used to determine the ROIs for subsequent communication runs. A standard convolution-based GLM analysis was performed on the localizer datasets using three boxcar functions for the three tasks. Using two different linear contrasts calculated from the fitted GLM beta weights, two ROls were automatically selected for each task: The first region was based on the main effects of the task (contrast: task vs. rest). The second region was more specific for the task as it was based on the balanced differential effects, e.g., for task 1 the contrast was selected as task 1 (+2), task 2 ($1)$, task $3(-1)$. The choice of the two contrasts was motivated by the need of jointly accounting for both the generalized activation evoked by the task (main effects independent of the other tasks) and the differential effects of each specific task relative to the other tasks.

The unsupervised selection procedure for each ROI from the GLM tmaps consists of multiple steps. Starting from the GLM map obtained by a given contrast (t statistics), the algorithm adaptively searches for a $t$ statistic threshold such that at least twenty voxels are selected forming one or more compact clusters of at least five voxels. A compact cluster is obtained from a set of adjacent voxels, where two voxels are considered adjacent if they are directly connected (i.e. they touch each other) at one of their faces, edges or vertices. Because the simple iterative search among many thresholds would imply a new search of all the clusters for each new threshold, to avoid repeating the search for all thresholds, we used an interpolation search method (Sedgewick, 1990). This algorithm searches for a given value in a sorted and indexed list with on average $\mathrm{O}(\log (\log (\mathrm{n}))$ comparisons and can be implemented with the following code (adapted from (Sedgewick, 1990)): 


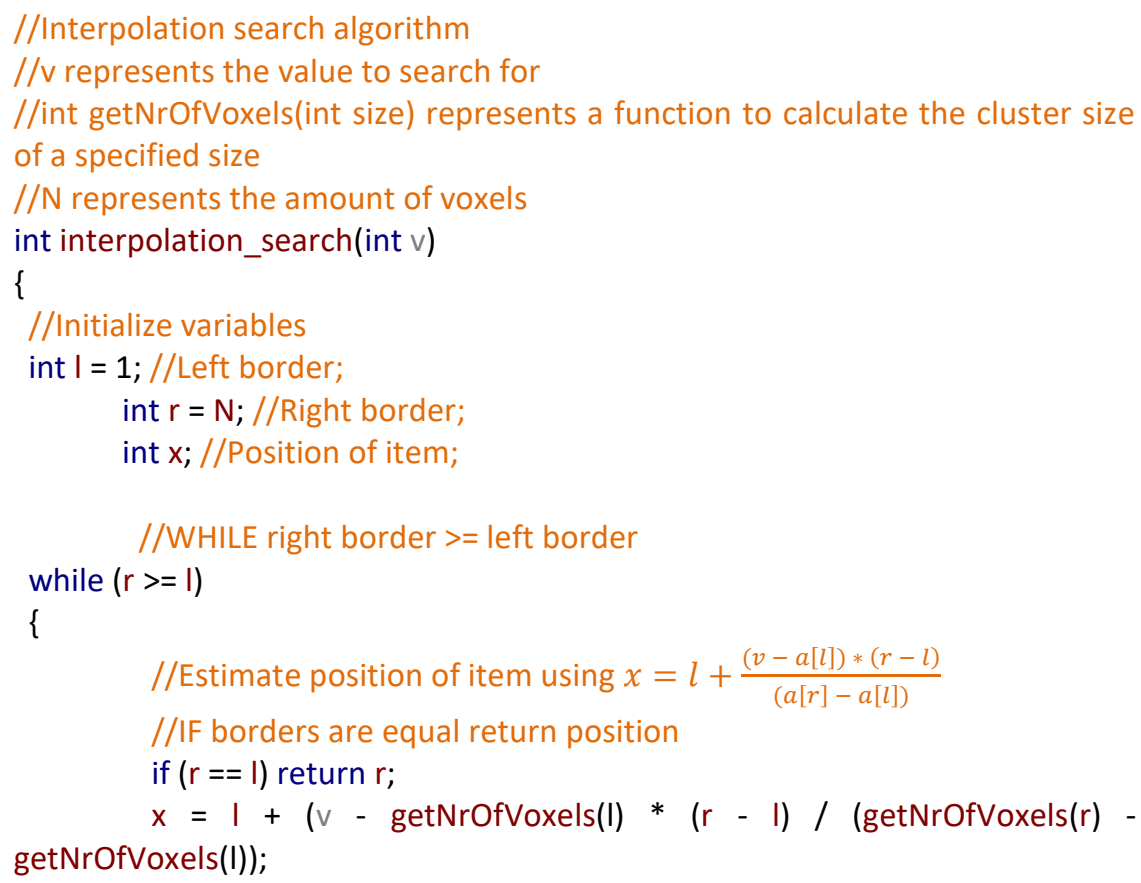

In this way, two sets of voxels (due to the above two contrasts) were independently determined for each task, albeit it was not prohibited that (i) more than one compact region was included in the final (multi-) ROI as a cluster assembly or network and (ii) all cluster assemblies ultimately consisted of the same voxels (in total or in part). 
Apart from ROI selection, the automated letter-decoding procedure was the same as used in the previous study, ranking all 27 characters according to their overall fit with a combined letter-decoding criterion incorporating both shape and amplitude-pattern information. The shape criterion is obtained by the correlation of the ROI time courses with a multi-filter bank of hemodynamic response functions; the amplitude pattern criterion is obtained by the regression of the ROI time-courses on the multiple hemodynamic response functions and the extraction of the estimated single-trial beta weights within all six ROls (Sorger et al., 2012). For calculating both shape and amplitude-pattern scores according to this criterion, the hemodynamic response functions could either be ideal response functions or individually derived empirical responses generated from the mean $\mathrm{ROI}$ time courses in the localizer run, thereby both response function types were explored in our tests. The ideal response functions were derived by linearly convolving the nine box-car functions ( 3 different onset delays $\times 3$ different stimulus durations) with the double-gamma hemodynamic response function (Friston et al., 1998).

To further investigate the potentials of the approach, especially in the direction of localizer-free experimental settings, the selected ROIs of each participant were transformed into Talairach space and combined into group-level (template) ROls. To test the performances of the GLMbased decoding using template ROls, a leave-one-out approach was used in which the spatially normalized (Talairach-transformed) ROls were merged from all participants except the participant that was investigated during decoding and then back-transformed to the native space of this participant and given as an input for the decoding algorithm.

All Talairach transformations were estimated on the individual anatomical images using 12-parameter affine transformations, and applied to the functional images.

In more detail, each anatomical data set was first transformed to the bicommissural (ACPC) space using a single affine transformation and then warped to Talairach standard space using a piece-wise grid-scaling affine transformation. The individual ROIs, initially obtained as voxel masks in the native space of functional data sets, were transformed to Talairach space using the same parameters obtained from the anatomical data sets after careful functional-to-anatomical image alignment. In order to 
avoid quality loss due to successive data sampling, functional-toanatomical image registration and normalization were performed in one single step by combining the estimated functional-anatomical affine transformation, the rigid-body ACPC affine transformation and the piecewise affine Talairach grid scaling. The merging of all individual ROls (both within and between subject) into a group-level (template) ROI was performed in the normalized space and consisted in the logical OR of all transformed masks. As the combined spatial transformations were also applied backward in one step and concatenated to form a single $4 \times 4$ affine transformation matrix, the obtained coordinates of the grouplevel (template) ROI voxels in the normalized space were used to calculate the coordinates of the group-level (template) ROI voxels in the original 3D space of each subject, allowing to precisely sample the data points in the functional data set via sinc interpolation.

\section{ICA-based Letter Decoding}

The novel ICA letter-decoding method consists of nine consecutive calculation steps to determine the encoded letter (figure 3). First, surface reconstruction is applied to the anatomical data set which was acquired for each participant at the beginning of each session. This step requires the correction of inhomogeneities of the MRI signal intensity across space, the white / gray matter border segmentation (performed with a region-growing method using intensity histograms), a number of morphological operations to smooth the borders of the segmented data and to separate the left from the right hemisphere, and the creation of a triangular mesh representing the white/gray matter border surface. This mesh is then projected into the functional data, thereby those voxels that lie within a standard range with respect of the white/gray matter border (usually from $1 \mathrm{~mm}$ inward to $3 \mathrm{~mm}$ outward) are tagged as "gray matter" voxels and define the gray matter masks to be used in the simulated real-time ICA analyses (for more details see (Formisano, Esposito, Di Salle, \& Goebel, 2004; Goebel, Esposito, \& Formisano, 2006)) (step one). GLM activation maps for each task were calculated from the functional localizer data by contrasting each task separately from each other compared to rest (step two). The resulting activation patterns for the three tasks were taken as whole-brain maps (without thresholds) and stored for the later selection step to determine (via spatial correlation with ICA component maps) which task was 
performed by the participant. In step three, the whole functional localizer data set is decomposed into spatial ICA components using offline ICA with the fastICA algorithm (Hyvarinen, 1999). Prior to the standard ICA decomposition, the initial dimension of the functional dataset is reduced to 40 using principal component analysis (PCA). The PCA stage is included in standard ICA methodology and the chosen final dimensionality corresponds to more than $20 \%$ of the time points, and accounts for more than $99.9 \%$ of the total variance-covariance, of the localizer data sets (Goebel et al., 2006). For each task multiple ICA components are selected based on the highest spatial correlation between the ICA components and the task-specific GLM maps from the previous step (step four). The selected ICA components' maps and representative time-courses are used as alternative maps for selection (see below) and to calculate empirical responses via trial-based averaging (step five). All analysis steps from one to five involving the anatomical and the localizer functional data sets were performed using BrainVoyager QX (v2.8.4 Brain Innovation B.V., Maastricht), and are not intended to be part of the final simulated real-time analysis. In fact, these steps could be in principle performed in a different session, separated from the communication runs. In our simulations, step one never took more than 10 minutes on a computer equipped with a quad core processor @3GHz and 8GB of RAM, and therefore can be completed by running a script during the calibration run (that lasts 12.4 minutes). Moreover, because calibration data can be preprocessed online, and the analysis in steps two and three are performed in the native space, step two (GLM on calibration data) and steps three, four and five (off-line ICA + component selection based on GLM maps + empirical response function generation) could be all completed in about 3 minutes on the same computer. This suggests that all preliminary calculations can be completed before the on-line run without excessive delay between the calibration and the on-line runs.

Steps from six to nine are part of the final simulated real-time analysis of the communication runs.

Step six pertains to the letter encoding process. For the real-time ICA, the time window was set to 40 time points (covering the duration of each letter encoding trial including the rest period between two successive trials) and the calculations were started at the end of each block. Because spatial smoothing improves the detection power of the 
ICA procedure (Esposito et al., 2003), a spatial smoothing with gaussian kernel of $6 \mathrm{~mm}$ FWHM is applied on-line to the functional data. No further pre-processing is done prior to the real-time ICA computation and a TxV data matrix is filled by taking the $\mathrm{V}$ voxel values corresponding to the $T$ time points in the current time window as soon as these are available for real-time processing. This matrix is fed into the real-time ICA algorithm.

In real-time ICA, the number of ICA components can be set to the number of time points of the data (Esposito et al., 2003). Although it would be possible to reduce the temporal dimensions of the data prior to ICA (as is normally done in off-line ICA), this reduction is not needed for real-time ICA due to the small number of time points. Conversely, it is more important (compared to off-line ICA) to use the entire variance/covariance of the data in the time window and extract as many ICA components as possible, to avoid missing some informative components in the data. In our simulations, after each completed letter encoding trial, 40 independent components were all available for subsequent processing (steps seven and eight) within about 4 seconds. After extraction, each ICA component time-course is used in a parallelized multiple linear regression analysis with nine standard hemodynamic reference functions. Thereby, letting $y(t)$ denote the column vector with a single component time-course, $X=\left[\mathbf{x}_{1}(t), \mathbf{x}_{2}(t), \ldots\right.$, $\left.\mathbf{x}_{9}(t)\right]$ the matrix with the nine reference functions as column vectors and $\mathbf{b}$ a column vector with nine unknown weights, the linear model $\mathbf{y}=\mathbf{X} \cdot \mathbf{b}$ is least-square fitted, and the coefficient of determination $\left(r^{2}\right)$ is obtained, for each component.

The standard hemodynamic reference functions could be either ideal (i.e. derived from the two-gamma hemodynamic response model (Friston et al., 1998)) or individually determined from the empirical responses in step five. Both response models were explored in this approach. After selecting the ICA component with the highest coefficient of determination $\left(r^{2}\right)$ (step seven), the spatial correlation coefficients between the corresponding ICA component map and the three GLM-based statistical maps from step three (or the ICA maps from step four) were calculated to identify which task was most probably performed during the letter-encoding trial (step eight). Finally, the most probable participant's letter choice was determined based on the results of the previous steps (step nine). 
Given the stochastic nature of the ICA algorithm, to investigate the reliability of the ICA results (Misaki et al., 2015) (i.e. the stability of the point of convergence), this analysis procedure was applied five times for each encoded letter with random initial conditions. In fact, the fastICA algorithm estimates the parameters of each component (component "weights") iteratively, returning a new component when the next iteration does not change the sum of squares of the weights more than a given tolerance (set by default to $10^{-6}$ ). Because these parameters are best initialized with random numbers, we wanted to check that repeating the simulations on the same data would not result in different decoded letters. In practice, because the important aspect in this context is not the exact point of convergence but the actual decoded letter, this step is not needed in the real-time application and therefore does not affect the on-line feasibility of the proposed approach.

Similarly to what has been done for the GLM approach, to further investigate the potential of the ICA approach with respect to a possible localizer-free experimental setting, the described algorithm was modified by removing steps one to three, and using a group-level GLM to generate a mean GLM map of all six participants (excluding the participant that was analyzed). To calculated this map, a fixed-effects GLM group data model was used to retain the highest statistical power in the studied group of participants. These template GLM maps replaced in this way the individual participant GLM maps in step eight. 


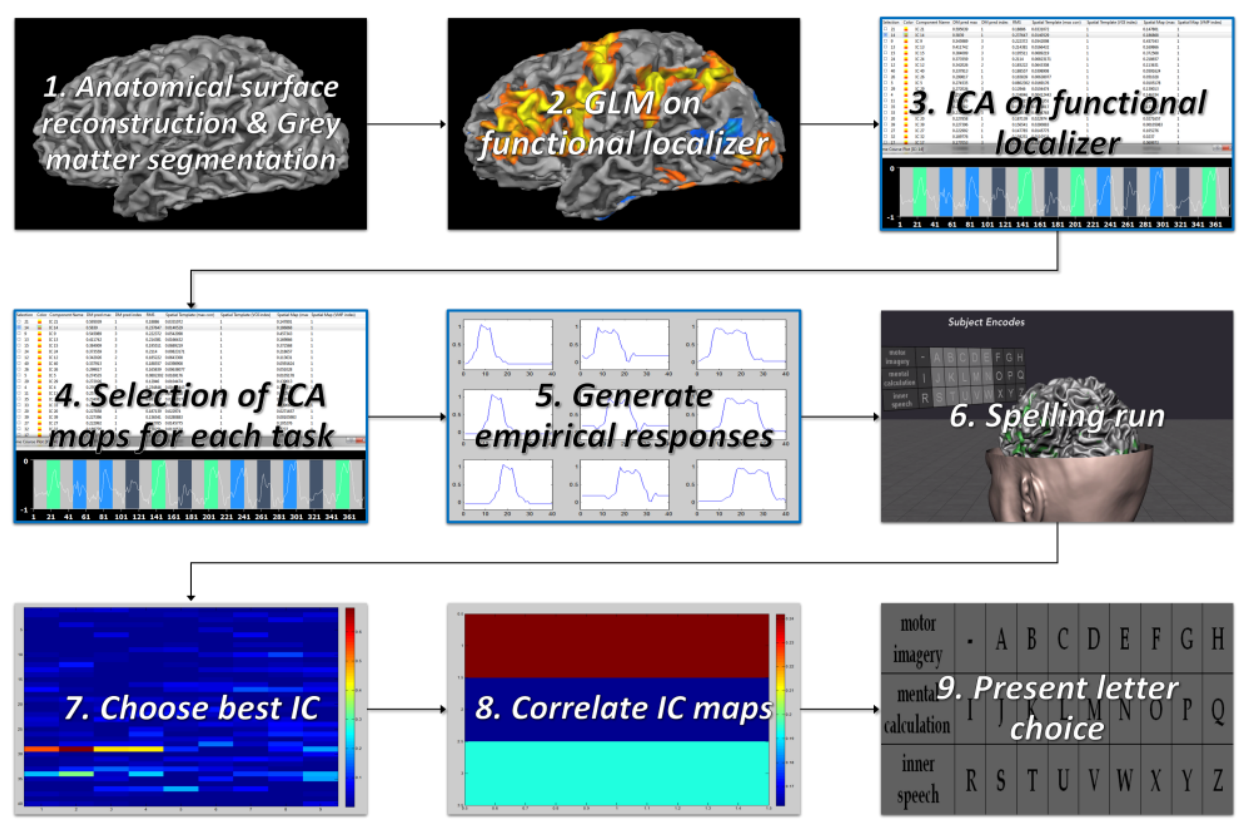

Figure 3: ICA letter decoding algorithm scheme. The ICA based decoding algorithm consists of nine iterative steps that are performed after each other. Steps three to five, marked with a blue border, are only necessary for implementing the empirical approach with real-time ICA. In a first step the anatomical surface reconstruction and a grey matter segmentation is performed to run ICA only on grey matter voxels. Afterwards reference GLM maps from the functional localizer run of each session are prepared. In step three the whole functional localizer is analyzed using ICA. For each task separate ICA maps are extracted based on the highest correlation with the GLM maps prepared in the previous step. These maps were later used in step eight. The following step generates empirical responses from the ICA time courses combined of each selected task in the prior step. After the preparation steps the real spelling run can be performed. Using the acquired single trial data for each letter the specific independent component is selected. In step eight the IC map of the selected component is correlated with the maps prepared in the localizer. The last step presents the letter choice to the experimenter. 


\section{Decoding Performance Evaluation}

In order to assess and compare the performances of the proposed solution, a similarity letter-based decoding criterion was introduced. In addition to the first (exact), second and third ranked letter choices (corresponding to constant chance levels of $3.7 \%, 11.11 \%, 55.55 \%$ ), an alternative decoding criterion was used where additional letter choices were considered among the four letters having the highest similarity (estimated in terms of the temporal correlation coefficient) compared to the ideal response function of the selected letter. The crosscorrelations coefficients of the nine different ideal response functions are shown in figure 4 . Thereby, if the letter choice were for example the letter ' $D$ ' the corresponding similarity criterion would additionally consider the letter choices ' $M$ ', ' $V$ ', ' $E$ ' and ' $B$ ', where ' $M$ ' and ' $V$ ' are the letters having a different task but the same response function, and the letter's ' $E$ ' and ' $B$ ' are based on the same task and have the two highest correlation coefficients across the nine response functions. If the response functions have the same correlation coefficients, the letter with the closest distance on the visual letter display is considered, e.g. if the letter choice were ' $S$ ', also ' $R$ ' would be selected but not ' $U$ '. This criterion produced a chance level of $55.55 \%$. For the performance evaluation according to this criterion, all the additional letters provided by the similarity criterion were considered equally possible alternative choices.

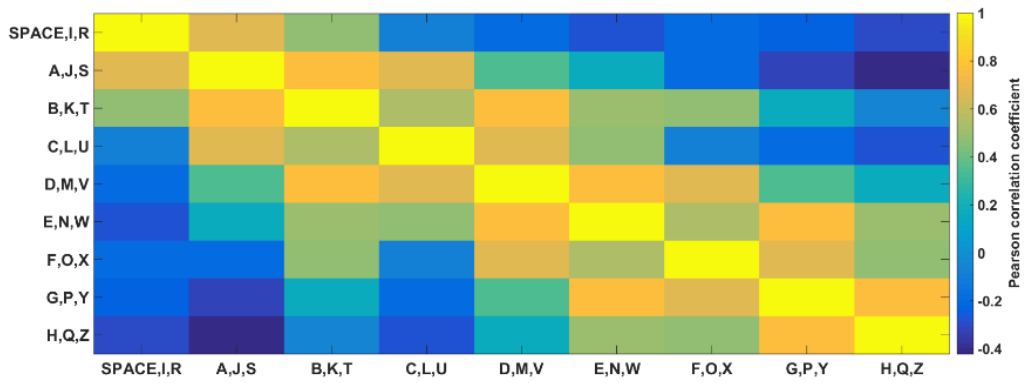

Figure 4: Correlation coefficients of the ideal response functions of each of the nine different on/offsets and lengths. For the similarity criterion the two letters having the highest correlation to the selected letter were accepted as correct choice. E.g. for letter choice "D" the letters " $E$ " and "B" would fulfil the criterion. The correlation coefficients show that letters that are very close on the letter display are not automatically the letters that have the highest correlation. 


\section{RESULTS}

\section{GLM Results}

Using the automated ROI selection approach on the GLM maps with empirical responses derived from the selected ROIs, a decoding accuracy of $77.66 \%$ for the first letter choice (chance level: $3.7 \%$, minimum accuracy $14.29 \%$, maximum accuracy $100 \%$, SD 23.31 ) was achieved by fully automating the selection based on the localizer session. Taking into account the first, second and third letter choice an accuracy of $92.75 \%$ was reached (chance level: $11.11 \%$, minimum accuracy $57.14 \%$, maximum accuracy $100 \%$, SD 15.36). Considering the similarity selection criterion gave a mean accuracy of $97.31 \%$ (chance level: $55.55 \%$, minimum accuracy $85.71 \%$, maximum accuracy $100 \%$, SD 4.37). The results of each single participant and word are presented in figure 5 .

With the ideal response functions, the mean accuracy was $76.81 \%$ for the first letter choice (minimum accuracy $14.29 \%$, maximum accuracy $100 \%$, SD 24.51), $91.38 \%$ for the first, second and third letter choice (minimum accuracy $70 \%$, maximum accuracy $100 \%$, SD 12.01) and $95.82 \%$ for the similarity selection criterion (minimum accuracy $85.71 \%$, maximum accuracy $100 \%$, SD 9.04).

Using the ROls combined from all participants (excluding the participant under investigation) resulted for the first letter choice in a mean accuracy of $35.94 \%$ (chance level: $3.7 \%$, minimum accuracy $0 \%$, maximum accuracy $77.78 \%$, SD 23.31), for the first, second and third letter choice a mean accuracy of $58.66 \%$ was reached (chance level: $11.11 \%$, minimum accuracy $28.57 \%$, maximum accuracy $100 \%$, SD 23.32 ). The similarity criteria resulted in a mean accuracy of $81.93 \%$ (chance level: $55.55 \%$, minimum accuracy $54.55 \%$, maximum accuracy $100 \%$, SD 14.58). 


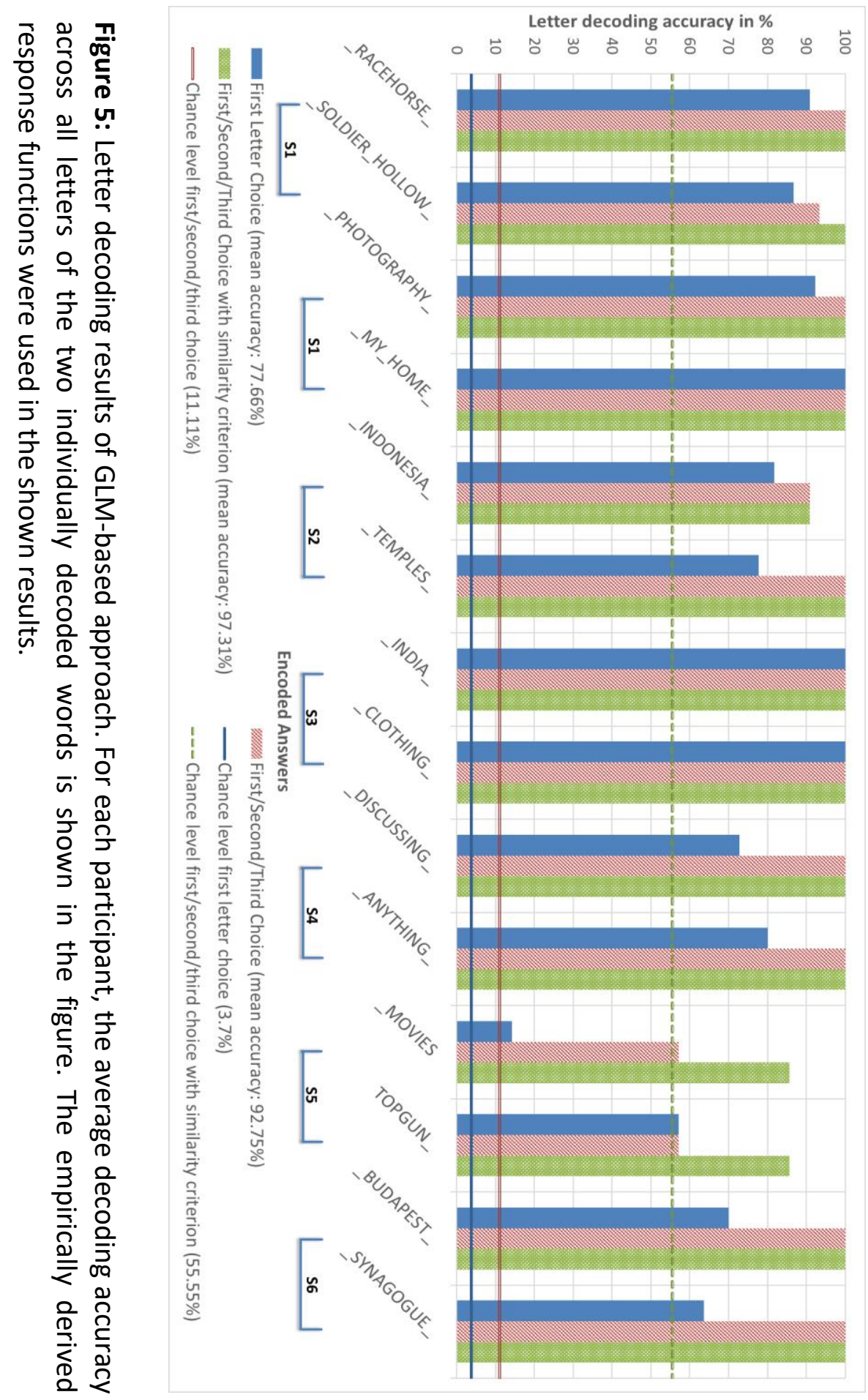




\section{ICA Results}

Using the proposed ICA approach, the letter choices of fourteen different words encoded by six healthy participants were decoded without (manually or automatically) defining ROls in advance.

For the first letter choice, a mean decoding accuracy was reached of $50.03 \%$ (chance level: $3.7 \%$, minimum accuracy $25.71 \%$, maximum accuracy $81.82 \%$, SD 16.65). For the first, second and third letter choices a mean decoding accuracy was reached of $67.06 \%$ (chance level: $11.11 \%$, minimum accuracy $31.43 \%$, maximum accuracy $87.27 \%$, SD 16.48). Considering the similarity selection criterion, a mean decoding accuracy was reached of $83.04 \%$ (chance level: $55.55 \%$, minimum accuracy $60 \%$, maximum accuracy $98.46 \%$, SD 14.04). The results of each single participant and word are presented in figure 6 . In all cases, the use of ideal hemodynamic reference functions and localizer GLM maps yielded better performances compared to using individual empirical responses and ICA maps created from the localizer (step three to five) (first letter choice: $45.04 \%$, first, second and third letter choice: $63.69 \%$, first, second, third and similarity criterion $80.28 \%$ ). Figure 8 shows the difference between the GLM-based localizer map and an ICAbased map selected for decoding, as well as their respective overlap, for two cases of correct decoding of a single letter, respectively in a participant with a good overall performance (and higher overlap for this case) and in a participant with a low mean performance (and lower overlap in this case).

Using not the individual participant GLM localizer maps but the template GLM localizer map obtained from the mean of all participants (except the one currently processed) produced an accuracy of $40.25 \%$ (chance level: $3.7 \%$, minimum accuracy $11.43 \%$, maximum accuracy $76.92 \%$, SD 17.71). For the first, second and third letter choice a mean accuracy of $54.06 \%$ was reached (chance level: $11.11 \%$, minimum accuracy $14.29 \%$, maximum accuracy $92.31 \%$, SD 22.76 ). The similarity criterion resulted in a mean accuracy of $76.53 \%$ (chance level: $55.55 \%$, minimum accuracy $28.57 \%$, maximum accuracy $100 \%$, SD 16.43 ).

An overview of the mean accuracies of both methods is shown in figure 7. 


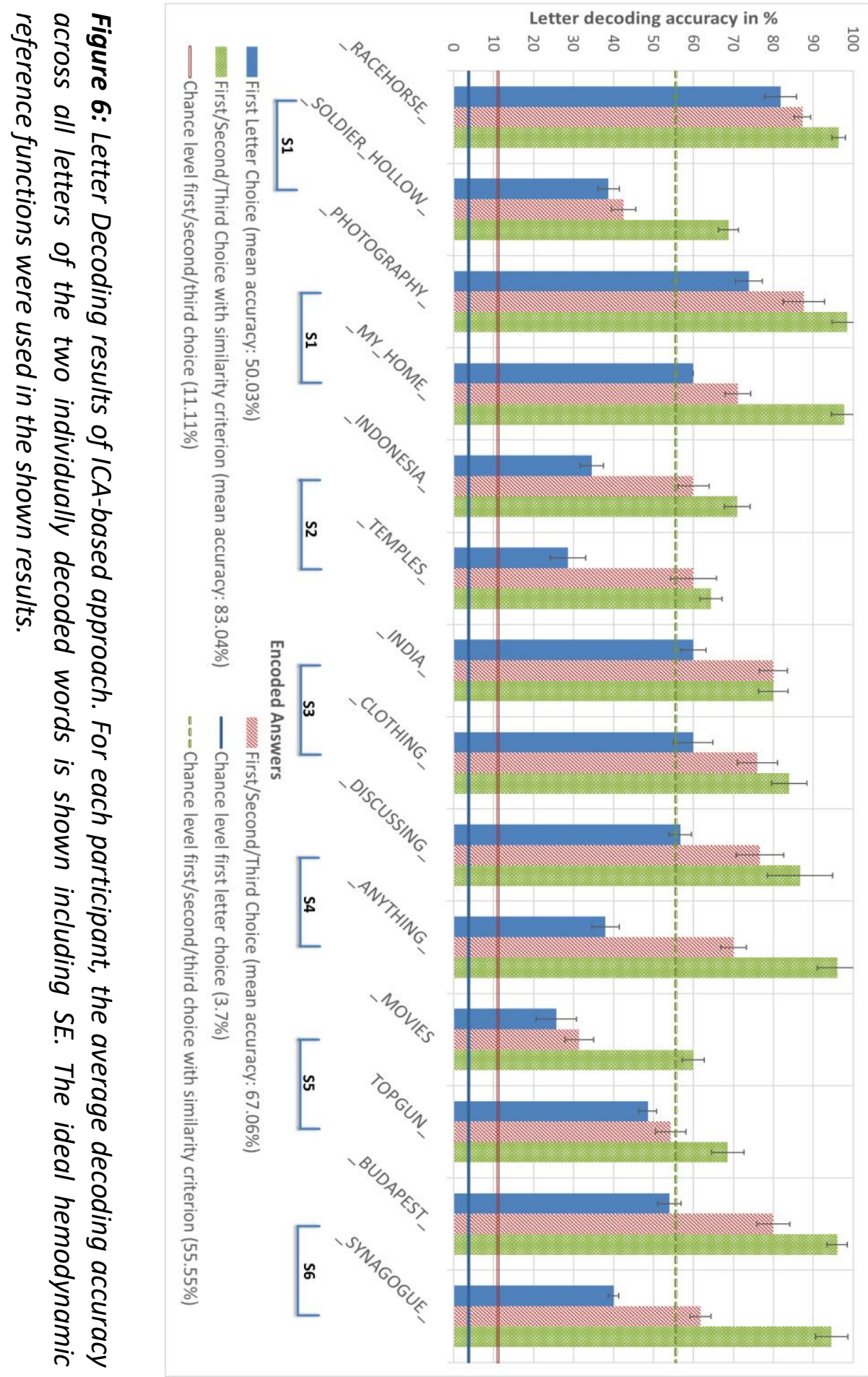




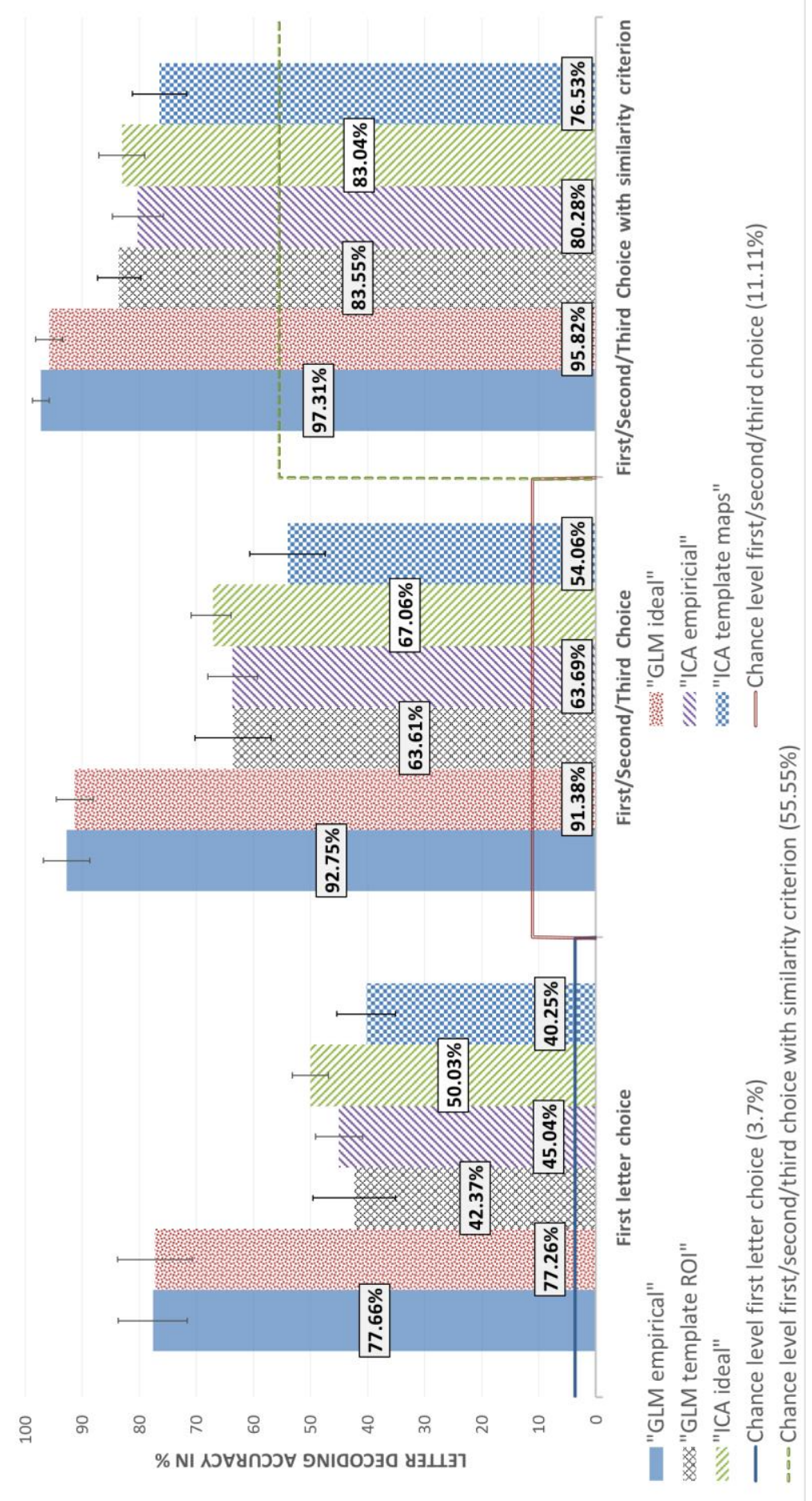

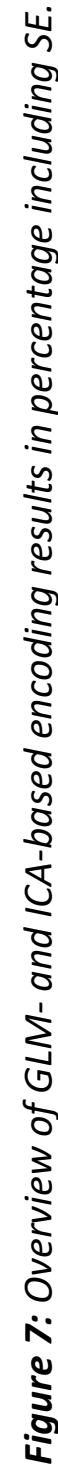




\section{DISCUSSION}

Here we have presented, and explored the performances of, a novel mechanism for automated GLM-based ROI selection and introduced the use of real-time ICA in fMRI-based motor-independent communication $\mathrm{BCls}$.

We had two main objectives in mind: (i) proposing an automated, yet stable and flexible, hard thresholding mechanism for univariate GLM modeling of localizer data sets for possible use in GLM-based letter-byletter decoding, without the need of cognitive network expertise related to the specific chosen mental tasks, and (ii) evaluating the decoding performances of a multi-dimensional data projection method like ICA that can work in real time but is not based on compact ROI preselection, and therefore avoids the setting of a voxel-level threshold.

As a target application for the introduced methods, we used the rt-fMRI communication $\mathrm{BCl}$ presented in [9]. In this $\mathrm{BCl}$ the subject encodes a single letter during each trial and the total time needed to spell a complete word can be up to 10-20 minutes (i.e. the number of letters in the word times the total trial duration including the rest period). As this is a common duration for a typical fMRI experimental session, in practice, this $\mathrm{BCl}$ can be used to respond to a single question (posed by the researcher) that accepts a single (long) word, or pairs of short words with a preposition or conjunction in between, as a possible answer.

\section{Accuracy of the automated GLM-based ROI selection}

The automated GLM-based ROI selection approach showed highly similar accuracies compared to those reached by the previous expertguided ROI selection approach (76\% vs $82 \%$ in the first letter choice (Sorger et al., 2012)). Note that this performance was obtained without the use of prior spatial (anatomical or functional) information about the location of the ROls. In fact, only geometric parameters are set for the ROI searching algorithm that were chosen empirically. More specifically, we found that selecting ROls with at least 20 voxels in each clusters produced stable results across all participants. This choice was inspired by previous work (Sorger et al., 2012), where a total of six ROls (two ROIs per task) were manually defined for supervised letter decoding, and each ROI comprised four contiguous voxels within a certain fMRI 
slice (thereby, the total number of voxels that were a priori selected for decoding was 24). In order to be in line with previous choice (and allow a better comparison between supervised and unsupervised approaches), we decided to set the minimum cluster size to 5 voxels and the minimum total number of activated voxels to 20 voxels. Because the ROIs are not manually defined in the unsupervised approaches (i.e. the ROIs can in principle appear in any location of the brain), we intended to be slightly more conservative for the minimum cluster size ( 5 instead of 4 voxels) and allow for potentially more clusters selected (4 instead of 2 clusters in the specific case of clusters with minimal size). In general, we believe this choice was not over-fitted to our data set because the initial choice was not based on specific calculations or statistics performed on the data sets but rather on a priori considerations made (and used) in previous work. On the other hand, when we used the same thresholds for a data set not included in the previous study (namely first session of S1), we obtained similar decoding performances compared to most of the other data sets. Future studies could systematically explore the effect of the number of selected voxels to determine more criterions for optimal static or dynamic voxel selection thresholds. In some individual cases, little adjustments of these parameters lead to better performances (not reported), suggesting that possible improvements in this procedure are still possible. Future work is needed to explore the impact and variability of these parameters on the automatic GLM decoding performances also in other information-encoding paradigms. We also found that, for this approach, using empirically derived hemodynamic reference functions should be preferred albeit the difference, compared to (Sorger et al., 2012), was rather small. This is still in line with earlier studies showing that there is a high variability of the single-trial BOLD responses across different individuals and different brain regions (Aguirre, Zarahn, \& D'esposito, 1998; Handwerker, Ollinger, \& D’Esposito, 2004).

To explore the performances of the GLM-based decoding in a hypothetical localizer-free experimental session, we also replaced the ROIs automatically obtained from the individual localizer data with a different set of ROls obtained from the localizer data of all other participants, however, the results based on these group-representative template ROIs were considerably worse than those obtained with individual localizers. This might be due to the small number of 
participants studied that could have determined a low degree of generalization of the calculated group ROls, or to the fact that using individual ROls always lead to better results and higher decoding accuracies in general. Further studies involving a considerably higher number of participants have to be performed in order to systematically investigate the potential of this approach. Also, the difference of subject S2 performing mental singing and not inner speech could have lead to a different network activity pattern and therefore to suboptimal results.

More in general, when a $\mathrm{BCl}$ or neurofeedback experiment makes use of predefined ROls, as in the reported template-based approach, the accuracy also relies on the quality of the registration between the functional data of the specific session and the anatomical or functional data in standardized space for the used ROIs. Thereby, the use of alternative registration algorithms, such as, e.g., cortex-based alignment (CBA) (Frost \& Goebel, 2012) or boundary based registration (BBR) (Greve \& Fischl, 2009) procedures, may result in different accuracies, and must be further investigated to get more insight about which registration procedure is most suitable for $\mathrm{BCl}$ and neurofeedback experiments.

As letters that appear close in the display (visual space) do not necessarily correspond to the most similar ideal responses (response space), we also introduced a novel similarity selection criterion to handle alternative choice options based on the measured BOLD responses. This criterion allowed reaching a mean accuracy of $97.31 \%$ across all participants and a maximum accuracy of $100 \%$ in some participants, against a chance of $55.55 \%$. Not only this criterion expands the potential use of the present $\mathrm{BCl}$, e.g., in possible combination with word prediction tools like the so called "Text on 9 keys" or "T9" used in mobile phones or similar solutions, but also allowed a more detailed investigation about how the task selection itself is affected by (slightly) wrong results in single steps of the procedure which would be otherwise not visible by only investigating the first choice results.

Although the results were overall encouraging, some variability in the performances were observed and for one of the experimental participants (S5), performances were substantially worse than the others. This variability could be in part explained by the fact that, among all participants, subject S5 was the only one with merely one previous $\mathrm{fMRI}$ session, whereas all the others had three or (many) more previous 
fMRI sessions (see Table 1). However, overall, we did not find a significant correlation between the letter performances and the number of previous $\mathrm{fMRI}$ sessions, suggesting that already two or three sessions could suffice for a subject to become accustomed with the $\mathrm{BCl}$. Nonetheless, a closer inspection of the results suggested that some of the selected $\mathrm{BCl}$ regions turned out to be not strongly enough related to any of the mental tasks during the encoding runs. Although this might be an isolated case, the ROI selection of areas completely unrelated to the areas initially expected to be involved in the mental task, occurred in another participant as well (albeit with less impact on the final performances), suggesting that the localizer run was probably suboptimal for both these participants and therefore this might be an important aspect, especially for the GLM-based decoding approach that strongly relies on the localizer data quality. In general, a participant needs to be able to perform the mental tasks well and reliably enough to allow sufficient statistical contrast among the different tasks and to ensure that the selection of the different ROls is sufficiently related to each task, otherwise additional quality measures and spatial guides for ROI selection procedures are needed. For instance, the effect of poor localizer performances on the ROI selection procedure could be potentially mitigated by combining the functional information available from the GLM with atlas-derived templates of the expected location for the brain regions. This could give a better generalization of the calculated beta weights in task-specific areas compared to other brain areas.

It would be in principle possible to replace the proposed GLM-based decoding with multi-voxel pattern analysis (MVPA) models and implement a categorical classification based on local multivariate patterns. However, this would not necessarily improve the communication performances when the errors are due to poor localizer results. In fact, MVPA techniques also rely on the selection of compact $\mathrm{ROI}(\mathrm{s})$ from the localizer session and additionally require long training sessions for multi-class classification (Stokes, Thompson, Cusack, \& Duncan, 2009), thereby, their application to a 27-class problem would easily become computationally prohibitive and would therefore make us depart from the main objective of simplifying and automatizing the present communication $\mathrm{BCl}$. 


\section{Accuracy of the real-time ICA approach}

With multivariate, not local, but whole-brain distributed, and fully datadriven approaches like ICA, the selection of specific brain areas for each task is not necessary, albeit the problem is shifted to the (real-time) selection of task-specific patterns. In this study a sliding window of 40 time points was used to estimate ICA components and to select the component of choice in the signal. It has been previously shown that whenever a task-related component is clearly dominant in the signal, this also results in comparable accuracy between real-time ICA and GLM (Esposito et al., 2003). However, at the end of our simulated real-time analysis, the average accuracy of the real-time ICA approach across all letters was overall lower compared to the GLM ( $\sim 50.03 \%$ compared to $\sim 77.66 \%)$. This can be possibly explained by the fact that the parameters of the experiment were purposely designed to strengthen the GLM decoding approach in the first place. For instance, using more mental tasks producing spatially non-overlapping patterns and less (or no) constrains on the timing control of the task performance, would possibly favor the ICA approach, given that the letter choice strongly relies on the spatial patterns in the first part of the pipeline. Nevertheless, the results clearly suggest that ICA performs significantly above chance level already for the first letter choices (50.03\%, chance level: $3.7 \%$ ), and can reach more than $80 \%$ success in combination with the similarity selection criterion (chance level: $55.55 \%$ ).

For this approach we did not find that, using empirically derived hemodynamic reference functions gives better performances as in the case of the GLM based approach. This can be explained by the fact that ICA component time course is not based on a single area but on a distributed network of areas, and this has the effect of reducing the variability of the single-trial BOLD responses due to the (weighted) averaging process implied by the real-time ICA decomposition (Aguirre et al., 1998; Handwerker et al., 2004).

Moreover, it turned out that only small benefit resulted from using individual localizer information in the ICA component selection compared to the GLM based method, and that using templates instead of individual localizers made ICA-based decoding slightly outperform GLM-based decoding. This indicates that while using localizer data remains the best option for reaching optimal performances with both the GLM- and ICA- decoding approaches, the ICA-based decoding 
approach seems to have higher potential for working with predetermined template maps in a possible localizer-free session. This is because the final selection of the best-decoding ICA component at each letter is mainly determined by the intrinsic signal-to-noise of the spatial pattern of activity and to a lesser extent by the precise overlap between the component and the localizer maps (as illustrated in figure 8), and this signal-to-noise depends on the trade-off existing between temporal (i.e., how many time points) and spatial (i.e., how many voxels) data dimensions for the ICA decomposition (Esposito et al., 2003).

In general, the temporal dimension of ICA decompositions is to be necessarily adapted to the single-block duration (in this case it was adapted to the single letter encoding). In fact, while shorter time windows do not necessarily impair the possibility to successfully decompose the data in real-time into meaningful spatial patterns, thereby providing the correct ROls for task selection via spatial correlation, the component time-course associated with the selected component will be necessarily truncated, and this will likely impair the response selection, which is based on temporal correlations. In line with previous work (Esposito et al., 2003), some preliminary simulations with a localizer data set (not shown) suggested that real-time ICA can often capture signal variations in time windows as short as the rising or falling time of the BOLD response, which is in the order of the time-to-peak of a typical hemodynamic response function (4-8s, 2-4 TRs). However, when the time window is set to include only a few time points, the latencies of the component time-course cannot be unambiguously decoded due to the absence of sufficient baseline (or plateau) points. It is therefore to be expected, also in different experimental designs, that the minimum duration of the time window for real-time ICA cannot be less than 20-25s, i.e. the minimum duration of a BOLD "impulse" response.

Other work on real-time ICA has mainly focused on the performances of different publicly available ICA algorithms applied in sliding window (Soldati, Calhoun, Bruzzone, \& Jovicich, 2013a) and different procedures to monitor in real-time a target component (Soldati, Calhoun, Bruzzone, \& Jovicich, 2013b). In both studies, real-time ICA was applied to simulated real-time $\mathrm{fMRI}$ experiments using real $\mathrm{fMRI}$ data. By comparing our current implementation to these works, we noted that the fastICA algorithm (Hyvarinen, 1999), that we currently use for real- 
time ICA, was found to be one of the four best performing algorithms among fourteen different algorithms (Soldati et al., 2013a). Moreover, among different procedures for selecting a target component of interest, a "back-projection" method deriving static spatial information from the functional localizer using off-line ICA, was found to outperform other approaches for dynamic component selection during the simulated real-time acquisition (Soldati et al., 2013b). This approach is very similar to our current implementation, as we also perform off-line ICA on the localizer data sets to generate three task-specific target components, to which the real-time components are spatially correlated.

Potential improvements of the ICA-based method are also possible in relation to the selection of the voxels to be included in the real-time calculations (before pattern generation and selection). In the present application, we performed a highly general selection by including all gray matter voxels, as this is known to improve spatial ICA decompositions in general (Formisano et al., 2004). Additional voxel selection schemes with a better balance between voxels related vs. voxels unrelated to the task pattern may further increase the performances, as it has been shown in an off-line ICA application (Aragri et al., 2006). In future applications, the use of atlas-derived sets of regions or network configurations might be used with the double purpose to define the global voxel space for ICA calculation on the one hand and allow better component selection on the other hand, resulting in more standardized and portable design for the $\mathrm{fMRI}-\mathrm{BCl}$, where the preparatory phase is ideally completed outside the scanner.

Moreover, in the present application, only task-related ICA components are considered for the automatic letter decoding. In future applications, other components classifiable on-line as specific noise or motion components according to general task-independent spatio-temporal features (see, e. g., (De Martino et al., 2007)) or via on-line estimation of motion parameters, could be useful for a combined real-time ICA-GLM approach where these "confound" components are regressed out before applying the described GLM approach. 


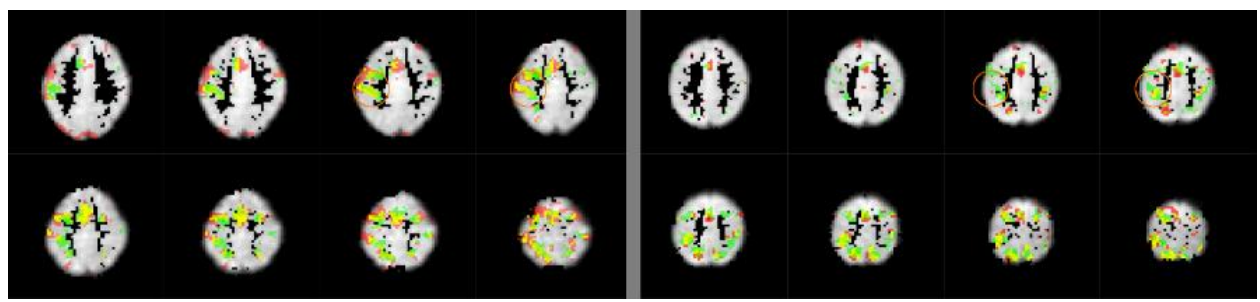

Figure 8: Maps of successful single-trial letter decoding results using ICA. Left panel: participant with high average accuracy (S1). Right panel: participant with low average accuracy (S5). The GLM-derived localizer map is overlaid in green, whereas the selected ICA map for this trial is overlaid in red. The overlap between localizer maps and ICA maps is overlaid in yellow. A circle highlights a brain region in the right prefrontal cortex where the selected ICA map overlaps well (S1) or not well (S5) with the GLM-based localizer map. 


\section{CONCLUSIONS}

We demonstrated that a higher level of automation for ROI-based GLM decoding techniques is possible using proper ROI-searching algorithms, and that real-time ICA can potentially reduce the amount and criticality of prior information needed for decoding spatially distributed patterns, in $\mathrm{fMRI}$-based communication $\mathrm{BCls}$.

There are three practical implications of this study: (i) whenever the localizer is available, the GLM-approach remains the decoding method of choice; (ii) the introduction of novel mechanisms for unsupervised thresholding of localizer maps may considerably reduce the amount of expertise and time spent on trimming the ROIs; (iii) the use of real-time ICA in localizer-free experimental settings can become an option, but, given the current gap observed in the performances between templatebased and localizer-based solutions, this could make sense only in particular cases where localizers are not possible because of extremely limited scanning time or critical for any reason, e.g., in the context of diagnostics in patients with disorders of consciousness or generally fast exhaustion of such patients.

Nonetheless, we believe that future research and development along these lines may lead to further increasing the level of automation of $\mathrm{fMRI}$-based $\mathrm{BCls}$. This aspect may turn out to be particularly relevant for brain-based communication when the ability to process cognitive stimuli of varying complexity is challenged by the complete absence of a behavioral response. More in general, motor impairment, mental fatigue, attention disorders and other cognitive problems typically contribute to the difficulty of communication, thereby more flexible and automated communication $\mathrm{BCls}$ might be helpful in restoring some communication ability. 


\section{ACKNOWLEDGMENTS}

The research leading to these results has received funding from the European Community's Seventh Framework Programme FP7/2007-2013 under grant agreement number PITN-GA-2011-290011. This work was also supported by the Netherlands Organization for Scientific Research (NWO; RUBICON 446-09-010 awarded to BS) and the European Research Council (ERC; advanced grant \#269853 awarded to RG). 


\section{REFERENCES}

Aguirre, G. K., Zarahn, E., \& D'esposito, M. (1998). The variability of human, BOLD hemodynamic responses. Neurolmage, 8(4), 360-369. https://doi.org/10.1006/nimg.1998.0369

Aragri, A., Scarabino, T., Seifritz, E., Comani, S., Cirillo, S., Tedeschi, G., ... Di Salle, F. (2006). How does spatial extent of fMRI datasets affect independent component analysis decomposition? Human Brain Mapping, 27(9), 736-746. https://doi.org/10.1002/hbm.20215

Cohen Kadosh, K., Luo, Q., de Burca, C., Sokunbi, M. O., Feng, J., Linden, D. E. J., \& Lau, J. Y. F. (2016). Using real-time fMRI to influence effective connectivity in the developing emotion regulation network. Neurolmage, 125, 616-626. https://doi.org/10.1016/j.neuroimage.2015.09.070

De Martino, F., Gentile, F., Esposito, F., Balsi, M., Di Salle, F., Goebel, R., \& Formisano, E. (2007). Classification of fMRI independent components using IC-fingerprints and support vector machine classifiers. Neurolmage, 34(1), 177-194. https://doi.org/10.1016/j.neuroimage.2006.08.041

Eklund, A., Andersson, M., Ohlsson, H., Ynnerman, A., \& Knutsson, H. (2010). A brain computer interface for communication using real-time fMRI. In Proceedings - International Conference on Pattern Recognition (pp. 36653669). https://doi.org/10.1109/ICPR.2010.894

Esposito, F., Seifritz, E., Formisano, E., Morrone, R., Scarabino, T., Tedeschi, G., ... Di Salle, F. (2003). Real-time independent component analysis of fMRI time-series. Neurolmage, 20(4), 2209-2224. https://doi.org/10.1016/j.neuroimage.2003.08.012

Farwell, L. A., \& Donchin, E. (1988). Talking off the top of your head: toward a mental prosthesis utilizing event-related brain potentials. Electroencephalography and Clinical Neurophysiology. https://doi.org/10.1016/0013-4694(88)90149-6

Formisano, E., Esposito, F., Di Salle, F., \& Goebel, R. (2004). Cortex-based independent component analysis of fMRI time series. Magnetic Resonance Imaging, 22(10 SPEC. ISS.), 1493-1504. https://doi.org/10.1016/j.mri.2004.10.020

Friston, K. J., Fletcher, P., Josephs, O., Holmes, a, Rugg, M. D., \& Turner, R. (1998). Event-related fMRI: characterizing differential responses. Neurolmage, 7(1), 30-40. https://doi.org/10.1006/nimg.1997.0306

Frost, M. A., \& Goebel, R. (2012). Measuring structural-functional correspondence: Spatial variability of specialised brain regions after macro-anatomical alignment. Neurolmage, 59(2), 1369-1381. https://doi.org/10.1016/j.neuroimage.2011.08.035

Goebel, R., Esposito, F., \& Formisano, E. (2006). Analysis of Functional Image Analysis Contest (FIAC) data with BrainVoyager QX: From single-subject to 
cortically aligned group General Linear Model analysis and self-organizing group Independent Component Analysis. Human Brain Mapping, 27(5), 392-401. https://doi.org/10.1002/hbm.20249

Greve, D. N., \& Fischl, B. (2009). Accurate and robust brain image alignment using boundary-based registration. Neurolmage, 48(1), 63-72. https://doi.org/10.1016/j.neuroimage.2009.06.060

Handwerker, D. A., Ollinger, J. M., \& D'Esposito, M. (2004). Variation of BOLD hemodynamic responses across subjects and brain regions and their effects on statistical analyses. Neurolmage, 21(4), 1639-1651. https://doi.org/10.1016/j.neuroimage.2003.11.029

Hyvarinen, A. (1999). Fast and Robust Fixed-Point Algorithm for Independent Component Analysis. IEEE Trans. Neur. Net., 10(3), 626-634.

Koush, Y., Meskaldji, D.-E., Pichon, S., Rey, G., Rieger, S. W., Linden, D. E. J., ... Scharnowski, F. (2015). Learning Control Over Emotion Networks Through Connectivity-Based Neurofeedback. Cerebral Cortex, bhv311. https://doi.org/10.1093/cercor/bhv311

Lorenz, R., Monti, R. P., Violante, I. R., Anagnostopoulos, C., Faisal, A. A., Montana, G., \& Leech, R. (2016). The Automatic Neuroscientist: A framework for optimizing experimental design with closed-loop real-time fMRI. Neurolmage, 129, 320-334. https://doi.org/10.1016/j.neuroimage.2016.01.032

Lorenz, R., Pascual, J., Blankertz, B., \& Vidaurre, C. (2014). Towards a holistic assessment of the user experience with hybrid BCls. Journal of Neural Engineering, 11(3), 35007. Retrieved from http://stacks.iop.org/1741$2552 / 11 / \mathrm{i}=3 / \mathrm{a}=035007$

Megan, T., Cohen, J. D., Lee, R. F., Norman, K. a, \& Turk-browne, N. B. (2015). Closed-loop training of attention with real-time brain imaging. Nature Neuroscience, 18(3), 1-9. https://doi.org/10.1038/nn.3940

Misaki, M., Barzigar, N., Zotev, V., Phillips, R., Cheng, S., \& Bodurka, J. (2015). Real-time $\mathrm{fMRI}$ processing with physiological noise correction Comparison with off-line analysis. Journal of Neuroscience Methods, 256(3), 117-121. https://doi.org/10.1016/j.jneumeth.2015.08.033

Naci, L., Cusack, R., Jia, V. Z., \& Owen, A. M. (2013). The brain's silent messenger: using selective attention to decode human thought for brainbased communication. J Neurosci, 33(22), 9385-9393. https://doi.org/10.1523/jneurosci.5577-12.2013

Naci, L., Monti, M. M., Cruse, D., Kübler, A., Sorger, B., Goebel, R., ... Owen, A. M. (2012). Brain-computer interfaces for communication with nonresponsive patients. Annals of Neurology, 72(3), 312-323. https://doi.org/10.1002/ana.23656

Nelder, J. A., \& Wedderburn, R. W. M. (1972). Generalized Linear Models. Journal of the Royal Statistical Society. Series A (General) Journal of the 
Royal Statistical Society. Series A (General J. R. Statist. Soc. A, 13517213(3), 370-384. https://doi.org/10.2307/2344614

Oldfield, R. C. (1971). The assessment and analysis of handedness: The Edinburgh inventory. Neuropsychologia. https://doi.org/10.1016/00283932(71)90067-4

Posse, S., Binkofski, F., Schneider, F., Gembris, D., Frings, W., Habel, U., ... Eickermann, T. (2000). A new approach to measure single-event related brain activity using real-time fMRI: Feasibility of sensory, motor, and higher cognitive tasks. Human Brain Mapping, 12(1), 25-41. https://doi.org/10.1002/1097-0193(200101)12:1<25::AIDHBM30>3.0.CO;2-H

Reuderink, B., Nijholt, A., \& Poel, M. (2009). Affective Pacman: A frustrating game for brain-computer interface experiments. In A. Nijholt, D. Reidsma, \& H. Hondorp (Eds.), Lecture Notes of the Institute for Computer Sciences, Social-Informatics and Telecommunications Engineering (Vol. 9 LNICST, pp. 221-227). Berlin, Heidelberg: Springer Berlin Heidelberg. https://doi.org/10.1007/978-3-642-02315-6_23

Ruiz, S., Buyukturkoglu, K., Rana, M., Birbaumer, N., \& Sitaram, R. (2014). Realtime $\mathrm{fMRI}$ brain computer interfaces: Self-regulation of single brain regions to networks. Biological Psychology, 95(1), 4-20. https://doi.org/10.1016/j.biopsycho.2013.04.010

Sedgewick, R. (1990). Algorithms in C. Boston, MA, USA: Addison-Wesley Longman Publishing Co., Inc.

Sokunbi, M. O., Linden, D. E. J., Habes, I., Johnston, S., \& Ihssen, N. (2014). Real-time fMRI brain-computer interface: development of a "motivational feedback" subsystem for the regulation of visual cue reactivity. Frontiers in Behavioral Neuroscience, 8(NOV), 392. https://doi.org/10.3389/fnbeh.2014.00392

Soldati, N., Calhoun, V. D., Bruzzone, L., \& Jovicich, J. (2013a). ICA analysis of fMRI with real-time constraints: an evaluation of fast detection performance as function of algorithms, parameters and a priori conditions. Frontiers in Human Neuroscience, 7(February), 19. https://doi.org/10.3389/fnhum.2013.00019

Soldati, N., Calhoun, V. D., Bruzzone, L., \& Jovicich, J. (2013b). The Use of a priori Information in ICA-Based Techniques for Real-Time fMRI: An Evaluation of Static/Dynamic and Spatial/Temporal Characteristics. Frontiers in Human Neuroscience, 7(March), 64. https://doi.org/10.3389/fnhum.2013.00064

Sorger, B., Dahmen, B., Reithler, J., Gosseries, O., Maudoux, A., Laureys, S., \& Goebel, R. (2009). Another kind of "BOLD Response": answering multiplechoice questions via online decoded single-trial brain signals. Progress in Brain Research, 177(C), 275-292. https://doi.org/10.1016/S0079- 


\section{3(09)17719-1}

Sorger, B., Reithler, J., Dahmen, B., \& Goebel, R. (2012). A real-time fMRI-based spelling device immediately enabling robust motor-independent communication. Current Biology, 22(14), 1333-1338. https://doi.org/10.1016/j.cub.2012.05.022

Stoeckel, L. E., Garrison, K. A., Ghosh, S., Wighton, P., Hanlon, C. A., Gilman, J. M., ... Evins, A. E. (2014). Neurolmage : Clinical Optimizing real time fMRI neurofeedback for therapeutic discovery and development, 5, 245-255. https://doi.org/10.1016/j.nicl.2014.07.002

Stokes, M., Thompson, R., Cusack, R., \& Duncan, J. (2009). Top-Down Activation of Shape-Specific Population Codes in Visual Cortex during Mental Imagery. Journal of Neuroscience, 29(5), 1565-1572. https://doi.org/10.1523/JNEUROSCI.4657-08.2009

Sulzer, J., Haller, S., Scharnowski, F., Weiskopf, N., Birbaumer, N., Blefari, M. L., ... Sitaram, R. (2013). Real-time fMRI neurofeedback: Progress and challenges. Neurolmage, 76, 386-399. https://doi.org/10.1016/j.neuroimage.2013.03.033

Weiskopf, N. (2012). Real-time fMRI and its application to neurofeedback. Neurolmage, 62(2), 682-692. https://doi.org/10.1016/j.neuroimage.2011.10.009

Yoo, S. S., Fairneny, T., Chen, N. K., Choo, S. E., Panych, L. P., Park, H., ... Jolesz, F. a. (2004). Brain-computer interface using fMRI: spatial navigation by thoughts. NeuroReport, 15(10), 1591-1595. https://doi.org/DOI 10.1097/01.wnr.0000133296.39160.fe 


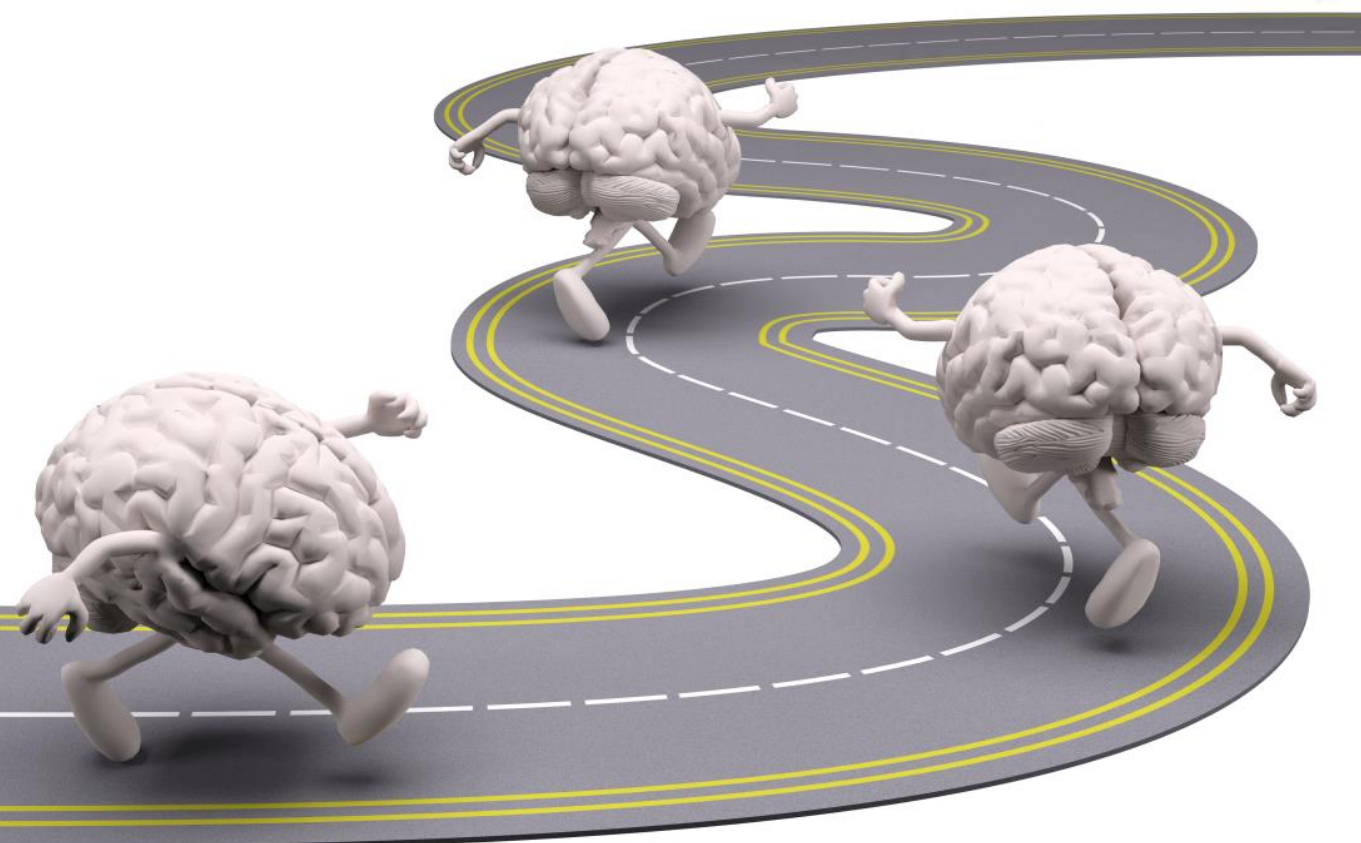




\section{Chapter 3}

\section{MR-Encephalography for $\mathrm{BCl} /$ neurofeed back applications with high temporal resolution sampling}

Corresponding publication:

Lührs, M., Riemenschneider, B., Eck, J., Benitez, A., Poser, B., Heinecke, A., Krause, F., Esposito, F., Sorger, B., Hennig, J., Goebel, R., MR-Encephalography for $\mathrm{BCl} /$ neurofeedback applications with high temporal resolution. Neurolmage, 2018, submitted. 


\section{ABSTRACT}

Real-time functional magnetic resonance imaging ( $r t-f M R I)$ enables the update of various brain-activity measures during an ongoing experiment as soon as a new brain volume is acquired. However, the recorded Blood-oxygen-level dependent (BOLD) signal also contains physiological artifacts such as breathing and heartbeat, which potentially cause misleading false positive effects especially problematic in brain computer interface (BCl) and neurofeedback (NF) setups. The low temporal resolution of standard echo planar imaging (EPI) sequences (which is in the range of seconds) prevents a proper separation of these artifacts from the BOLD signal. MR-Encephalography (MREG) has been shown to provide the high temporal resolution required to unalias and correct for physiological fluctuations and leads to increased specificity and sensitivity for mapping task-based activation and functional connectivity as well as for detecting dynamic changes in connectivity over time. By comparing a standard EPI sequence and an MREG sequence for three different experimental fMRI paradigms (perception of house and face stimuli, motor imagery, Stroop task), the potential of this novel technique for future $\mathrm{BCl}$ and $\mathrm{NF}$ applications was investigated. First, adapted general linear model pre-whitening which accounts for the high temporal resolution in MREG was implemented to calculate proper statistical results and be able to compare these with the EPI sequence. Furthermore, the respiration- and cardiac pulsation-related signals were successfully separated from the MREG signal using independent component analysis which were then included as regressors for a GLM analysis. Only the MREG sequence allowed to clearly separate cardiac pulsation and respiration components from the signal time course. It could be shown that these components highly correlate with the recorded respiration and cardiac pulsation signal using a respiratory belt and fingertip pulse plethysmograph. Temporal signal-to-noise ratios of EPI and MREG were comparable. Functional connectivity analysis using partial correlation showed a reduced standard error in MREG compared to EPI. Also, direct time course comparisons by down-sampling the MREG signal to the EPI temporal resolution showed lower variance in MREG. In general, we show that the higher temporal resolution is beneficial for fMRI time course 
modeling and this aspect can be exploited in off-line application but also, is especially attractive, for real-time $\mathrm{BCl}$ and $\mathrm{NF}$ applications. 


\section{INTRODUCTION}

Recent technological advances in computational power and the advent of more sophisticated image reconstruction techniques over the last years made it possible to use higher spatial and temporal resolutions in functional brain imaging with MRI (Feinberg et al., 2010; Poser \& Setsompop, 2018; Rabrait et al., 2008). However, these new possibilities also require more advanced preprocessing and statistical computations, such as handling auto-correlations across many volumes, to fulfill the requirements for the commonly used parametric statistical analyses (Fadili, Ruan, Bloyet, \& Mazoyer, 2003). Specific advancements in the field of functional magnetic resonance imaging ( $\mathrm{fMRI}$ ) provided new acquisition methods such as simultaneous multi-slice (SMS, also known as multi-band, MB) (Feinberg et al., 2010; Feinberg \& Setsompop, 2013), blipped controlled aliasing (CAIPI) EPI blipped-CAIPI-EPI) (Moeller et al., 2010; Setsompop et al., 2012), echo volumar imaging EVI) (Rabrait et al., 2008), or simultaneous multi-slice inverse imaging (SMS-InI) (Hsu et al., 2017), allowing to acquire fMRI data using repetition times (TR) in the range of hundreds of milliseconds. The method used in this article to perform $\mathrm{fMRI}$ is called MR-Encephalography (MREG) (Hennig, Zhong, \& Speck, 2007). It is of particular interest for real-time applications because it allows to use a very high temporal resolution of $100 \mathrm{~ms}$ (and possible even up to $25 \mathrm{~ms}$ ). To be more precise, the higher temporal resolution is a crucial factor to increase sensitivity, specificity and signalto-noise ratios (SNR) with respect to confounding signals, as physiological noise can be separated from the signal of interest (Assländer et al., 2013; Feinberg \& Setsompop, 2013; Feinberg \& Yacoub, 2012; Lee, Zahneisen, Hugger, LeVan, \& Hennig, 2013; Lewis, Setsompop, Rosen, \& Polimeni, 2016; Lin et al., 2012; Posse et al., 2013; Zahneisen et al., 2011). This is possible since the high temporal resolution allows to record these confounding signals and with this gives the possibilty to remove these components from the signal of interest. Additionally, since most sequences that allow sub-second TRs are also suitable for real-time fMRI data analysis (possible image reconstruction within a TR), MREG should be regarded as a potential candidate in realtime setups. MREG combines the sampling efficiency of non-Cartesian trajectories with under-sampled variable density read-outs to achieve ultra-fast fMRI acquisition (Assländer et al., 2013; Hennig et al., 2007; 
Zahneisen et al., 2011, 2012). In conjunction with recent parallel receiver arrays this permits repetition times below $100 \mathrm{~ms}$ for wholebrain fMRI. This approach trades off some spatial resolution and introduces susceptibility artifacts in the presence of strong offresonance gradients to achieve the ultra-fast read-out (Assländer et al., 2013). The clear downside of highly-undersampled non-Cartesian acquisition is their significantly higher computational burden compared echo-planar acquisitions. As a consequence, for MREG there is currently no solution to fully reconstruct each frame in real-time (i.e., in less than $100 \mathrm{~ms})$. Even strong cluster computers are not able to fulfill the criteria of reconstructing data within a single TR. To overcome this computational issue, a novel real-time MREG (rtMREG) method was recently introduced, which uses minimal reconstructions and creates a time course of interest from preselected brain areas using individual reconstructions vectors (LI, 2014; Riemenschneider, Levan, Reisert, \& Hennig, 2015). With this approach, a real-time access of predefined areas is possible.

In the current study, we demonstrate the potential of MREG for realtime applications such as brain-computer interfaces ( $\mathrm{BCl}$ 's) and, more specifically, neurofeedback (NF). To be able to identify specific differences between MREG and standard EPI-sequence parameters, we conducted three different experiments, each of them twice in a counterbalanced fashion across participants: Once with a standard SMS-EPI sequence using a TR of $2000 \mathrm{~ms}$ and once using an MREG sequence with a temporal resolution of $100 \mathrm{~ms}$. This allowed to directly investigate the individual differences in single subjects as well as group differences using the two sequences. In addition, we examined the overall differences of both sequences with respect to co-registration of anatomical and functional data as well as preprocessing and analysis methods. A first analysis focused on the statistical properties of SMS-EPI and MREG data using the standard general linear model (GLM), which is commonly used in fMRI data analyses (Monti, 2011). A second analysis focused on the temporal signal to noise ratio (tSNR) of SMS-EPI and MREG data. Eventually, a third analysis explored the potentials of high temporal resolution acquisitions with respect to functional connectivity analysis. We aimed to show the direct benefit of MREG for real-time applications along the preprocessing and analysis pipeline, since this method was not yet applied in a $\mathrm{BCl}$ environment. Even the possibility to 
provide NF in a common (e.g., thermometer; (Krause et al., 2017) fashion, but use MREG with this very high temporal resolution will certainly have benefits for offline analysis as we will show in this article.

\section{Proposed real-time study setup}

Due to the computationally extensive image reconstruction time of MREG it is not possible to analyze a functional localizer within the current session. A dedicated functional localizer session is advised, unless only anatomical information will be used, to define a target region. When opting for a functional target region of interest (ROI) localizer session on a different day, as described below, the data needs to be analyzed offline before the actual real-time session. This way, potential signal shifts, which are characteristic for any k-space trajectory, are already accounted for in the extracted target regions. On both scanning days, anatomical reference datasets need to be acquired for the co-registration of the ROls. On the second day, the previously localized ROls can be aligned to the current position of the subject using a transformation matrix created by registering the anatomical datasets from day one and day two as shown in Figure 1. 


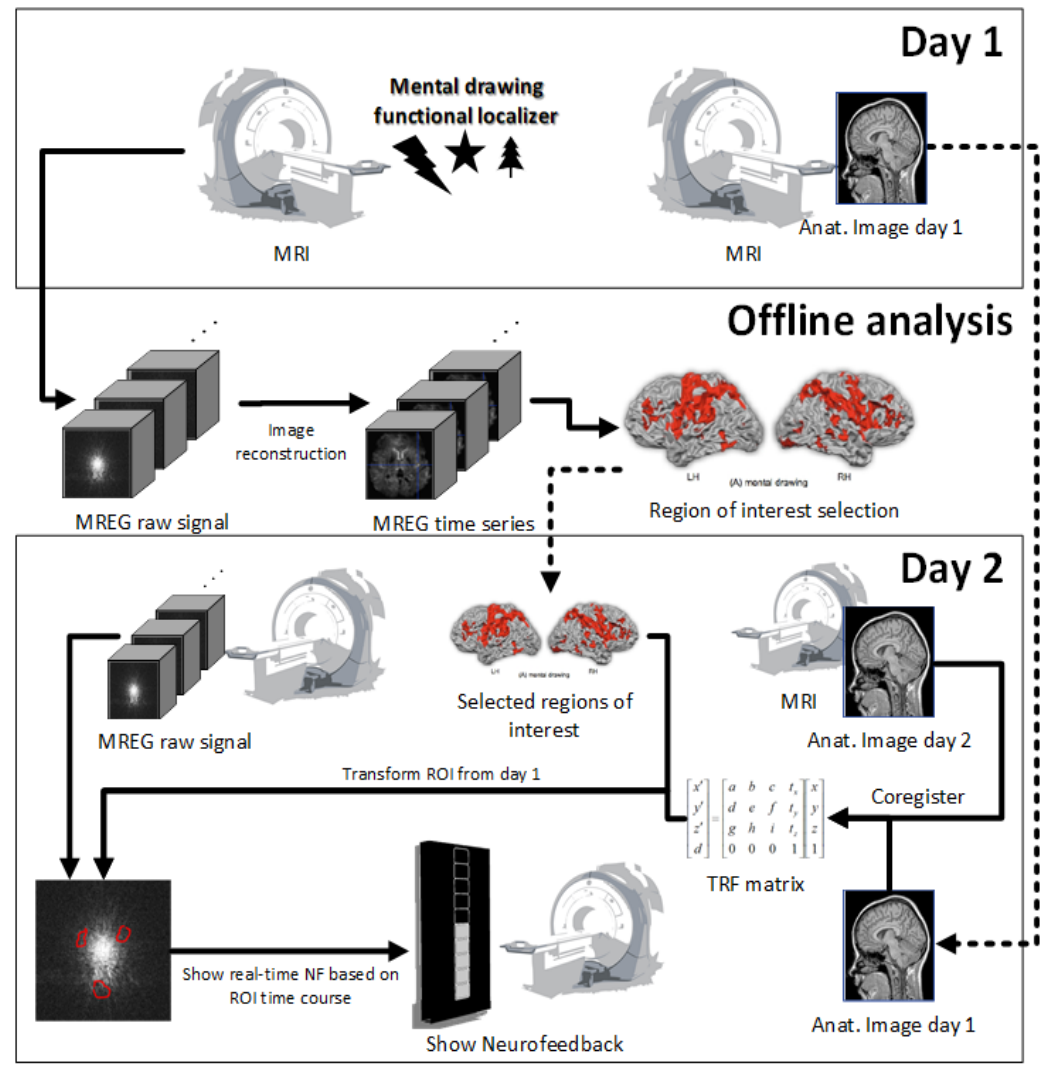

Figure 1: Workflow of rtMREG experiments. During the first day a localizer and anatomical scan is performed. At day two another anatomy is scanned for corregistration and localizing the region defined on the first day.

The matrix of the ROI-specific targeted partial reconstruction can be pre-computed right at the beginning of the actual real-time session and will then be applied for NF purposes during subsequent functional runs. The magnitudes of the raw data are used as a source for the NF calculations. The reconstruction results can be exported in real-time using e.g., a direct TCP/IP stream, which avoids potential delays possible when using a usual file transfer. To be able to receive the data directly a re-routing (e.g., 'tunneling') from the image reconstruction computer through the MRI console computer needs to be integrated, which can be realized by port forwarding from the image reconstruction computer to the real-time processing computer via the MRI console. 


\section{METHODS}

In this section we will cover the methods used to point out the differences between SMS-EPI and MREG data with respect to future real-time processing and the differences introduced by the high sampling rate.

\section{Participants}

Twelve healthy volunteers (six females; all recruited at Maastricht University, Maastricht, the Netherlands) aged between 23 and 39 years (mean $=28 ; \mathrm{SD}=4.97$ ) participated in the three experiments. All participants had normal or corrected-to-normal vision and had no history of neurological or psychological disorders. The volunteers were informed about the details of the study and the two different sequences. The study was approved by the local ethics committee, and participants gave their written informed consent before participating in the study.

\section{Study design}

All participants performed the same three tasks in pseudo-randomized order. The first task was a motor imagery paradigm consisting of eight task and nine rest blocks each lasting 24s. During the task blocks the participants were asked to draw a simple figure or object in their mind using the right hand (Lührs, Sorger, Goebel, \& Esposito, 2017; Sorger, Reithler, Dahmen, \& Goebel, 2012). Objects were for example stars, trees or cars that were easy to imagine for each person individually. The second task was a passive viewing task where the participants had to focus on the center of the screen while pictures of faces or houses were shown (Kanwisher, McDermott, \& Chun, 1997). Picture blocks had alternating faces and houses every $500 \mathrm{~ms}$, with a total duration of $16 \mathrm{~s}$. The rest blocks in between the picture blocks lasted $16 \mathrm{~s}$ as well. The third paradigm was a standard color-based Stroop task (Peterson et al., 1999). Participants had to press either the index finger for red colored text or the middle finger if the text color was green, while ignoring the semantic meaning of the text ("RED" or "GREEN) which was presented in the center of the screen. The slowest reaction time was set to $1.5 \mathrm{~s}$ and randomized stimulus interval ranging from $500 \mathrm{~ms}$ to $16 \mathrm{~s}$. 
Each run lasted 400 seconds for SMS-EPI and MREG. Each experiment was performed twice, once with SMS-EPI sequence and once with MREG. The whole experiment took approximately one hour for each participant including an additional anatomical scan.

\section{MRI data acquisition}

All data were acquired using a 3T MRI full body scanner (PRISMA fit, MR_VE11C, Siemens Healthcare, Erlangen, Germany) equipped with a 64-channel head coil. All sessions were scanned with the same scanning parameters for all participants. For the EPI data a multiband sequence (Feinberg et al., 2010; Moeller et al., 2010; Setsompop et al., 2012) was used where the scanning parameters were as follows: 200 volumes, $\mathrm{TR} / \mathrm{TE}=2000 \mathrm{~ms} / 30 \mathrm{~ms}$, flip angle 77 degrees, matrix size $=64 \times 64$ using a $192 \times 192 \mathrm{~mm}$ field of view (FOV), multiband factor 2, 50x 3.0-mm slices without gap. The MREG acquisition covered the exact same FOV as the EPI, with an isotropic nominal resolution of $3 \mathrm{~mm}$ resulting in a matrix size of $64 \times 64 \times 50, T R=100 \mathrm{~ms}$, flip angle of $23^{\circ}$, for 4000 volumes. We used a single-shot spherical stack-of-spirals trajectory for the read-out, as described in (Assländer et al., 2013). The reference scans were based on a gradient echo sequences (GRE) used for anatomical reference and calculation of the sensitivity maps for the MREG reconstruction had the following parameters: $T R / T E=381 \mathrm{~ms} / 2.46 \mathrm{~ms}$, flip angle 25 degrees, matrix=128 $\times 128$ using a $192 \times 192 \mathrm{~mm}$ field of view (FOV), 100x 0.5$\mathrm{mm}$ slices without gap.

Physiological signals (pulse oximetry, and respiratory depth) were recorded using the MR system's built-in wireless fingertip pulse plethysmograph and respiratory belt at $50 \mathrm{~Hz}$. For each participant, a MPRAGE T1-weighted anatomical data set encompassing the whole brain was acquired following the localizer experiment (scan parameters: $\mathrm{TR}=2250 \mathrm{~ms}, \mathrm{TE}=2.21 \mathrm{~ms}, \mathrm{FA}=9^{\circ}, \mathrm{FOV}=256 \times 256 \mathrm{~mm} 2$, matrix size $=$ $256 \times 256$, number of slices $=192$, slice thickness $=1 \mathrm{~mm}$, total scan time $=8 \mathrm{~min}$ and $26 \mathrm{~s}$ ).

EPI-SMS and MREG data preprocessing included motion correction and temporal filtering using BrainVoyager 20.6 (Brain Innovation B.V., Maastricht, Netherlands). For the MREG data an additional dynamic off resonance correction (DORK; (Pfeuffer, Van Moortele, Ugurbil, Hu, \& Glover, 2002) using FMRIB Software Library (FSL; http://www.fmrib.ox.ac.uk/fsl) was performed. A temporal high-pass 
filter of three cycles per time course was applied. The high-pass filtering was performed on the native data before the transformation to Montreal Neurological Institute (MNI) space to remove low frequency drifts from the data. In addition, linear trends were removed. No spatial smoothing was applied.

\section{MREG co-registration}

Unlike MREG, GRE data is relatively easy to co-register to individual anatomical datasets. For this reason, we first acquire a brief GRE reference scan shortly before MREG data is acquired and co-register GRE data to the anatomical dataset. Due to the temporal proximity of both MREG and GRE scans, the resulting transformation matrix can be then used on the MREG data.

More specifically, first the GRE data was stored as an fMRI (fmr) dataset in BrainVoyager. This format allows automatic boundary based registration (BBR) (Greve \& Fischl, 2009) of the functional and anatomical reference. Using this automatic procedure all GRE datasets were mapped to each subject's individual anatomical dataset in native space. After co-registration of the GRE images, the resulting transformation parameters were used to map the MREG data to the anatomical image. After transforming the MREG data into MNI standard space, the functional coverage was verified by overlaying all functional runs of all twelve participants on top of the MNI Colin27 standard template (Holmes et al., 1998). We also applied the BBR corregistration procedure to the SMS-EPI data to have maximally comparable results. The quality of the latter was also verified using the method described above, i.e., transforming the data into the $\mathrm{MNI}$ space and overlay all functional runs on top of the MNI Colin27 standard template.

\section{General linear model analysis}

fMRI data is most commonly analyzed using a general linear model (GLM; (Fadili et al., 2003). To ensure correct GLM analysis of the MREG data, global assumptions of model and noise (e.g., independence of repeated measurements over time (Barker, Aarabi, \& Huppert, 2013)) were re-evaluated. Since both sequences measured BOLD-related signals, we further investigate only the possibly violated assumptions that were caused by the higher sampling rate of MREG (Hao, Khoo, von Ellenrieder, \& Gotman, 2017; Uga, Dan, Sano, Dan, \& Watanabe, 2014). 
One assumption of the GLM is that there is no autocorrelation in the residuals (Eklund, Friman, Andersson, \& Knutsson, 2011). However previous work has shown that, this assumption is not always met (K. J. Friston et al., 2000). There are different sources causing the temporal autocorrelation in fMRI, e.g., trends, scanner imperfections or physiological noise (Boynton, Engel, Glover, \& Heeger, 1996; Eklund et al., 2011). As for the latter, since heartbeat and respiration are clearly visible in the MREG data, we were able to model them as confounds. The beta estimates are not directly affected by autocorrelation, but the serial correlation can result in biased estimates of the error variance and this can lead to inflated test statistic (Tak \& Ye, 2014).

To remove these correlations, the $\mathrm{fMRI}$ time series needs to be preprocessed. Here, we only focus on one of the most common processes, namely the pre-whitening, which should also perform best in block based stimuli paradigms (Friman \& Westin, 2005). To whiten the residuals, multiple steps need to be performed and different models can be used for the pre-whitening method, like auto-regressive (AR), moving-average (MA) or auto-regressive-moving-average models (ARMA, ARIMA or "Box-Jenkins") (Locascio, Jennings, Moore, \& Corkin, 1997). As the AR model is very often used in fMRI analysis, we decided to use this method (Rogers, Katwal, Morgan, Asplund, \& Gore, 2010).

Due to the high sampling rate of MREG data, higher order AR models may be required to sufficiently remove autocorrelation from the residuals. The AR model of a higher order $p$ can be described as:

$$
x(t)=\alpha_{1} x(t-1)+\ldots+\alpha_{p} x(t-p)+e(t)
$$

The parameter $e(t)$ is the white noise and parameters $\alpha_{1}, \ldots, \alpha_{p}$ are the AR parameters to be estimated for the different model orders (Eklund et al., 2011).

A model order needs to be selected objectively. This was done by selecting the model that minimizes an information criterion function. The Bayesian information criterion was used in this study:

$$
B I C(P)=-2 L L+P \ln (n)
$$

The BIC consists of the following parts: $\mathrm{P}$ is the model order; LL is the log-likelihood of the model fit; and $\mathrm{n}$ is the number of time points (Barker et al., 2013). It has been shown that this criterion worked well 
for fNIRS analysis (Barker, Rosso, Sparto, \& Huppert, 2016; Uga et al., 2014) which has a similar temporal resolution as MREG.

After the selection of the $A R(p)$ order the AR parameters was estimated using Burg's maximum entropy method. Although very often used, we decided not to use the Yule-Walker approach because it may lead to incorrect parameter estimates in case of nearly periodic signals. Instead, we used Burg's method (Burg, 1975; De Hoon, Van Der Hagen, Schoonewelle, \& Van Dam, 1996; Kay \& Marple, 1981), which is a recursive algorithm aiming to finding a sequence of values so that it constitutes the (empirical) partial autocorrelation function that is also described as reflection coefficients (Pollock \& Green, 1999).

The estimated AR parameters are then used to whiten the time series and model predictors using the following equation (Eklund et al., 2011):

$$
w(t)=x(t)-\sum_{i=1}^{p} \hat{\alpha}_{i} x(t-i)
$$

Here $\mathrm{p}$ is the order of the AR model and $\hat{\alpha}_{i}$ are the model parameter estimates.

To further investigate the relation to the explained variance in the model we conducted GLM analysis with and without additional confound predictors. More details about the added confounds can be found in the following section.

\section{Adding confound predictors using independent component analysis}

A suitable tool to improve the GLM model is to add additional predictors which only explain non-task relevant information of the data, so called confound predictors. These predictors can be generated using mathematical models or by extracting information from the data itself, provided that it is not part of the underlying paradigm used in the study. The BOLD fMRI signal arises from a complex mixture of neuronal, metabolic and vascular processes, and it is further corrupted by multiple non-neuronal fluctuations of instrumental, physiological (including motion) and subject-specific (psychophysiological) origin. Particularly, motion-related and physiological noise fluctuations are the main noise components of the signal, and data-driven multivariate approaches, such as principal and independent component analysis (PCA and ICA, 
respectively), strongly benefit from the information available in the shared task-independent phases of multi-voxel time courses. This in turn allows to robustly separate the spatially structured (noise) components from the data sets. After the extraction, the components need to be first classified as either signal or noise, thereby, a denoised fMRI time-series is usually reconstructed based only on the independent components classified as signal.

As a viable alternative, here we used ICA to create and add confound predictors to the GLM, in a sort of hybrid GLM-ICA analysis (see, e. g., (Mckeown et al., 1998)). This was done by first running the FastICA algorithm (Hyvärinen, 1999) on a given $\mathrm{FMRI}$ data set as implemented in BrainVoyager (ICA plugin), and then selecting the component time courses as candidate confounds for the GLM. When applied to the multivariate $\mathrm{fMRI}$ time-series, the FastICA algorithm allows to extract (up to) a predefined number of independent components, which can then be classified as either task-related or non-task-related signal sources (De Martino et al., 2007; McKeown et al., 1998) according to the correlation coefficient with the GLM task predictors. As we were only interested in non-task-related components, which do not (or only minimally) explain the multivariate (co)variance according to the experimental paradigm, each ICA component time course was correlated with the task predictor time courses and was only selected as a confound predictor in the GLM if the absolute value of the correlation coefficient was below the mean absolute correlation of all components or lower than 0.2. Given the high temporal dimensionality of the MREG time-series, up to ten ICA components were extracted from each run of each participant using the deflation version (i.e., one by one) of the FastICA algorithm, both for the SMS-EPI and MREG sequence. This approach ensured the computational feasibility of the procedure, even for future real-time applications (see, e. g., (Lührs et al., 2017)), because spatial maps are not used for reconstruction and the selected confound predictors are by definition not correlated to the GLM predictors (see, e. g., (McKeown, 2000; McKeown, Hansen, \& Sejnowsk, 2003). To verify that the extracted components included heartbeat and respiration signal information, it was correlated to the acquired physiological data using the fingertip pulse plethysmograph and respiratory belt. 


\section{Temporal signal to noise comparison}

To compare the overall signal quality, we conducted a comparison of the signal-to-noise ratio of the SMS-EPI and MREG data. This ratio can be seen as a comparison of the global signal level related to noise were the global signal is comprised of baseline and activation periods (Murphy, Bodurka, \& Bandettini, 2007; Welvaert \& Rosseel, 2013). This gives an indication of the overall signal quality compared to the background noise. Additionally, temporal filtering was applied using Gaussian full width half maximum kernel of $2 \mathrm{~s}$ low pass filter. This way the cleaned time course only consisted of the information of interest. Because this also inflates the noise term correlations of the GLM, we applied the filtering only to run a tSNR comparison between MRGE and SMS-EPI data. The following equation was used:

$$
t S N R=\frac{\bar{S}}{\sigma_{N}},
$$

where $\bar{S}$ is the average voxel time course and $\sigma_{N}$ the standard error of the mean.

The resulting tSNR maps contain a statistical difference between all SMS-EPI runs of all twelve participants compared to all MREG runs of all participants further described in the results section. For MREG data, TSNR values were computed using filtered and unfiltered data in order to see the effects of temporal filtering.

\section{Functional connectivity analysis}

The functional connectivity analysis has become more popular in recent years in real-time fMRI applications (Bastos \& Schoffelen, 2016; Karl J. Friston, 2011; Hutchison et al., 2013; Zilverstand, Sorger, Zimmermann, Kaas, \& Goebel, 2014). The connectivity between different brain regions can be used to strengthen functional connections to support rehabilitation processes (Canuet, 2015; Díez-Cirarda et al., 2017; Ochmann et al., 2017) or can give different insights than standard univariate analysis. Having a higher temporal resolution can be beneficial, e.g., for dynamic functional connectivity in event-based paradigms as shown by (Sahib et al., 2018) and gives more reliable connectivity results (Karahanoğlu \& Van De Ville, 2017). We investigated the potential benefits of a sequence with higher temporal resolution 
using both, simulated and real data (Akin, Lee, Hennig, \& LeVan, 2017). For the former, a partial correlation between two simulated regions of interests was modeled first. This was done using a double gamma hemodynamic response functions (HRF) (Glover, 1999) for each simulated region and convolving it with a box car function according to the block design protocol. Then, white noise was added to both simulated regions to mimic a fMRI signal. A control region was formed by only a white noise signal with zero mean. The partial correlation of the two simulated regions controlled for the white noise only signal was repeated 1000 times to model the difference in connectivity shape. This step was performed using (1) a temporal resolution of $2 \mathrm{~s}$ simulating fMRI TR and $100 \mathrm{~ms}$; (2) a temporal resolution of $100 \mathrm{~ms}$ simulating the MREG sampling rate. The results of this analysis can be found in section 3.4. To verify the results of the simulated data we also extracted time course information from the motor imagery experiment of all participants. We selected left sensorimotor (LSM) and left premotor (LPM) areas based on the SMS-EPI or MREG Stroop experiment run respectively and used a control region in white matter to control for global noise and physiological artifacts. Selecting the region in a different run is important to avoid misleading statistics caused by double dipping.

\section{Temporal down sampling of MREG to EPI resolution}

To be able to compare both signals in a direct application as a signal source for NF applications, the MREG time course was down sampled to the temporal resolution used for the SMS-EPI sequence (2s). The down sampling was performed by decreasing the sample rate of the MREG data by keeping the first sample and then every 20th sample after the first. Similar to the functional connectivity analysis the time course of LPM was extracted in both MREG and SMS-EPI for all participants in the motor imagery experiments.

\section{RESULTS}

\section{GLM and ICA analysis}

The GLM results mainly focus on the estimated AR model orders and the effect of adding additional confound predictors using ICA. 


\section{ICA confound predictors}

ICA was used to obtain additional confound predictors that were added to the GLM analysis. As shown in Figure 2 the respective ICA components for respiration and cardiac pulsation significantly correlated with the recorded cardiac pulsation and respiration data. For example, the respiration data for participant P07 gave a Pearson correlation of $r=0.4943$ with the respective ICA component and for the heartbeat a Pearson correlation of $r=0.89$ for the specific ICA component. Both were significant $(p<0.001)$.

Figure 2 depicts the ICA component maps for cardiac pulsation (red) and respiration (blue), as well as their respective time course are shown, together with the respective reference time course of the respiratory belt and fingertip pulse plethysmograph. 


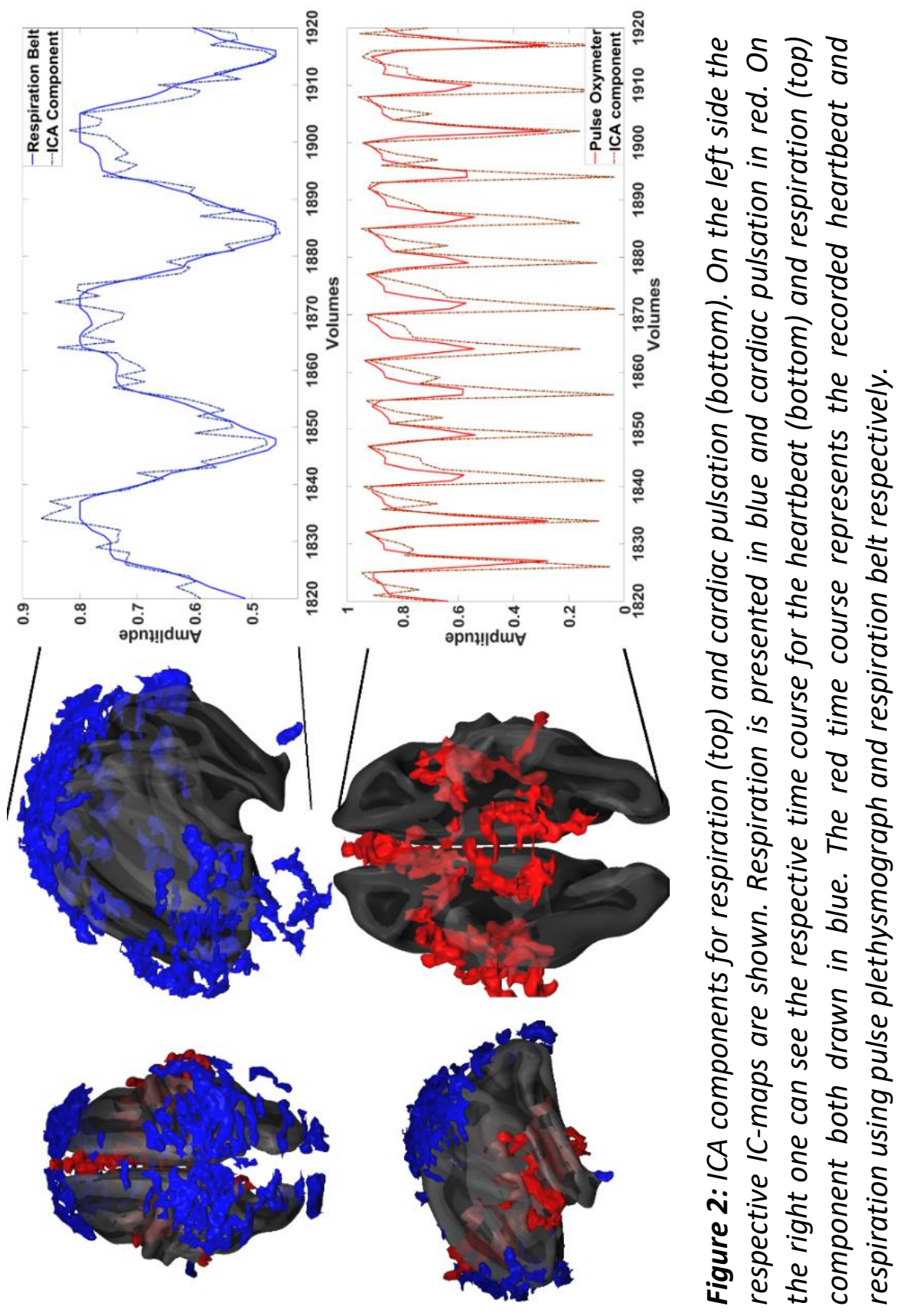




\section{GLM AR orders}

For all MREG experiments, BIC analysis estimated an average AR order across runs and participants of 21.30 without incorporating the ICA confound predictors. If the confound predictors were incorporated the estimated AR order was reduced to 17.97. Comparing individual AR order maps of each voxel for all participants showed a significant decrease of the estimated autocorrelation order $(p<0.05)$ in $61.32 \%$ of all voxels and a t-value below 0 (comparing MREG including ICA confound > MREG without confounds) in $97.8 \%$.

To illustrate the effect of the statistical inflation using no or low models for the autocorrelation correction, different AR models were used. Statistical maps for the motor imagery task are presented for subject P04 in Figure 3. 


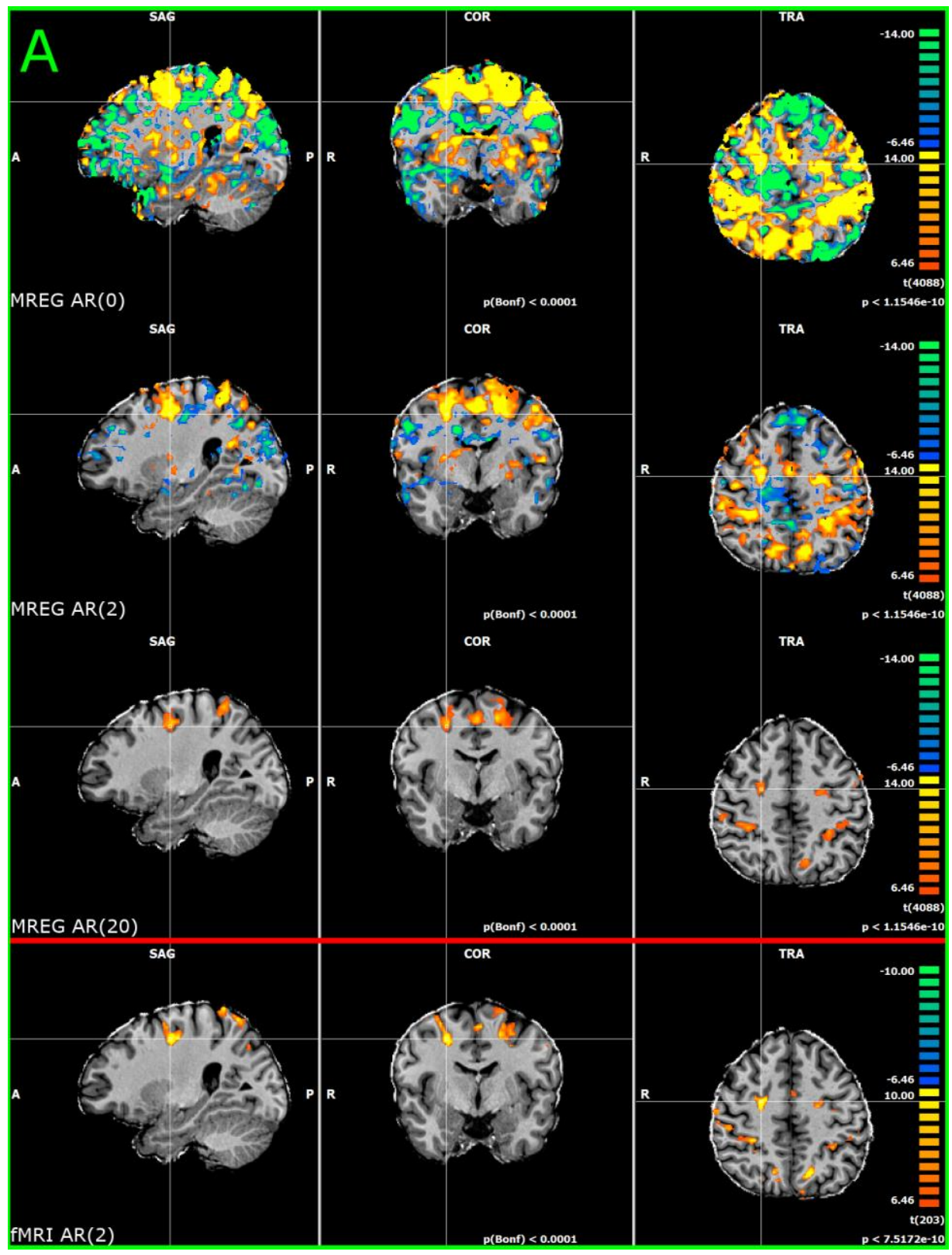

Figure 3: MREG AR correction using different AR orders. A) AR correction of standard GLM residuals without additional confound predictors (only motor imagery > rest). Different orders were used to investigate the effect of proper AR correction. The higher the order the lower the calculated t-values. B) AR correction of GLM including additional 


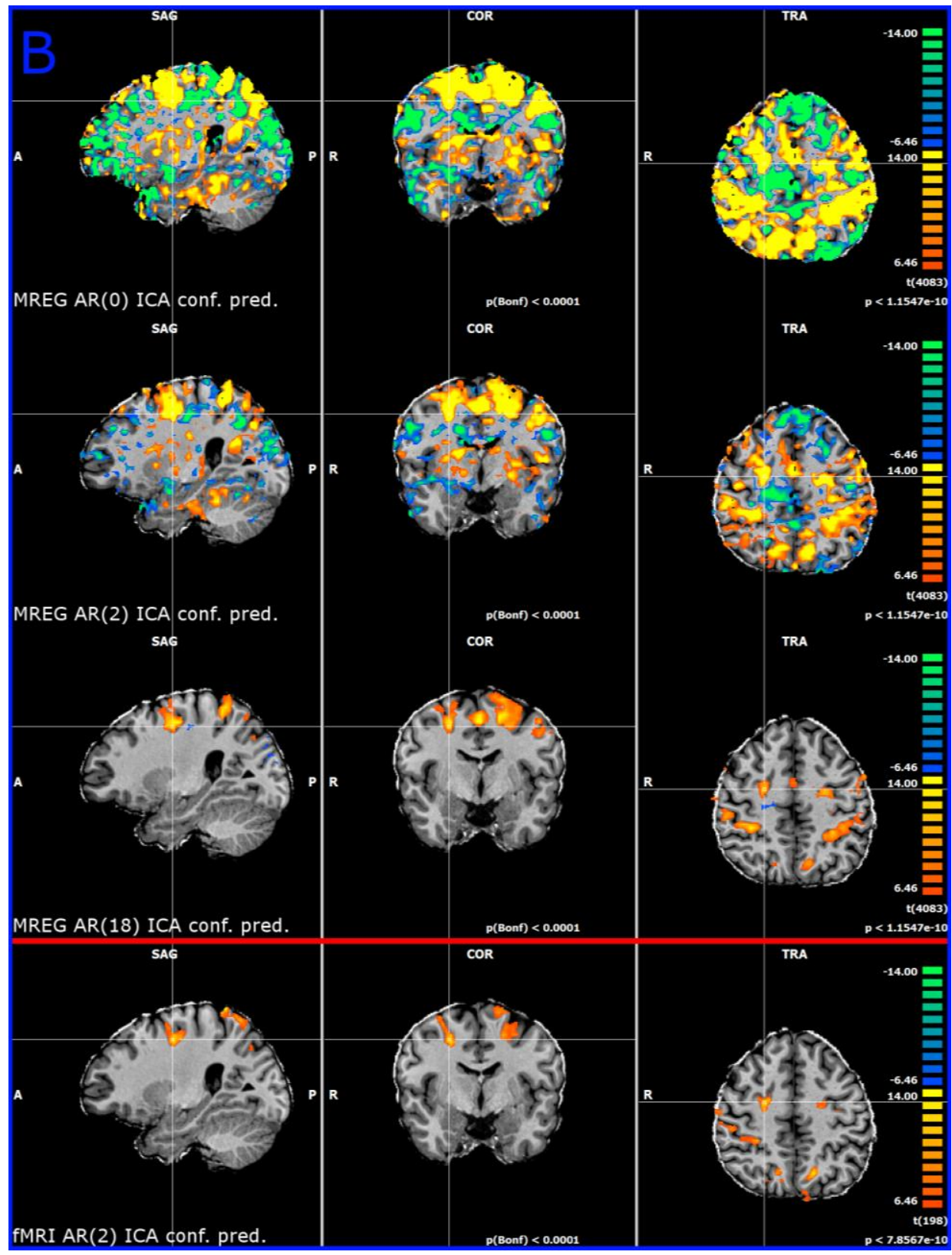

confound predictors for heartbeat and respiration extracted using ICA. The $t$-values are overall higher compared to the maps shown in part $A$. In the bottom of the figure a standard SMS-EPI analysis result is shown as a reference, once with (B) and once without (A) additional ICA based confound predictors. 
As shown in Figure $3 \mathrm{~A}$, no additional confound predictors were used and only the t-statistics of motor-imagery vs. rest are presented (Bonferroni corrected at $\left.p_{\text {bonf }}<0.0001\right)$ using different $A R$ orders $(0,2,20)$. Figure $3 B$ shows additional ICA confound predictors were added and the $\mathrm{t}$ statistics of the contrast motor-imagery $>$ rest is shown (Bonferroni corrected at $\left.p_{\text {bonf }}<0.0001\right)$ for different AR orders $(0,2,18)$. The high AR orders (20 and 18$)$ differ because they were estimated using BIC. Also, in individual participants this decrease is not significant $(p>0.05)$ across individual voxels. At the bottom of Figure 3, the standard SMS-EPI analysis results are shown using a standard $A R(2)$ model, with $(A)$ and without ICA confound predictors (). Unlike for SMS-EPI data, B). MREG data shows an increase in t-statistics when adding additional confound predictors. To show this effect in a specific volume of interest, we performed a ROI GLM analysis on the motor imagery run of P07 $10 \mathrm{~mm}$ radius cube centered at $\mathrm{MNI}$ coordinate: $6,-5,57$; Right BA 6, primary motor cortex). For MREG, the explained variance increased from $50.84 \%$ to $72.76 \%$. This effect was smaller for SMS-EPI data, which increased from $63.20 \%$ to $71.74 \%$. Here the t-values decreased when adding the ICA confound predictors to the GLM. Overall the decrease in statistical inflation using higher $A R(p)$ orders can clearly be seen when looking at the different t-maps. The t-values for SMS-EPI and MREG data were both Bonferroni corrected $\left(p_{\text {bonf }}<0.0001\right)$.

A detailed time course plot of the selected ROI is shown in Figure 4 which includes the model and error terms, as well as autocorrelation functions (ACF) for no AR correction and $A R(2)$ correction. The ACF of the residuals using the estimated $A R$ based on BIC was a nearly flat line and therefore not included in the plot because of no visible differences in the lags of the ACF. 


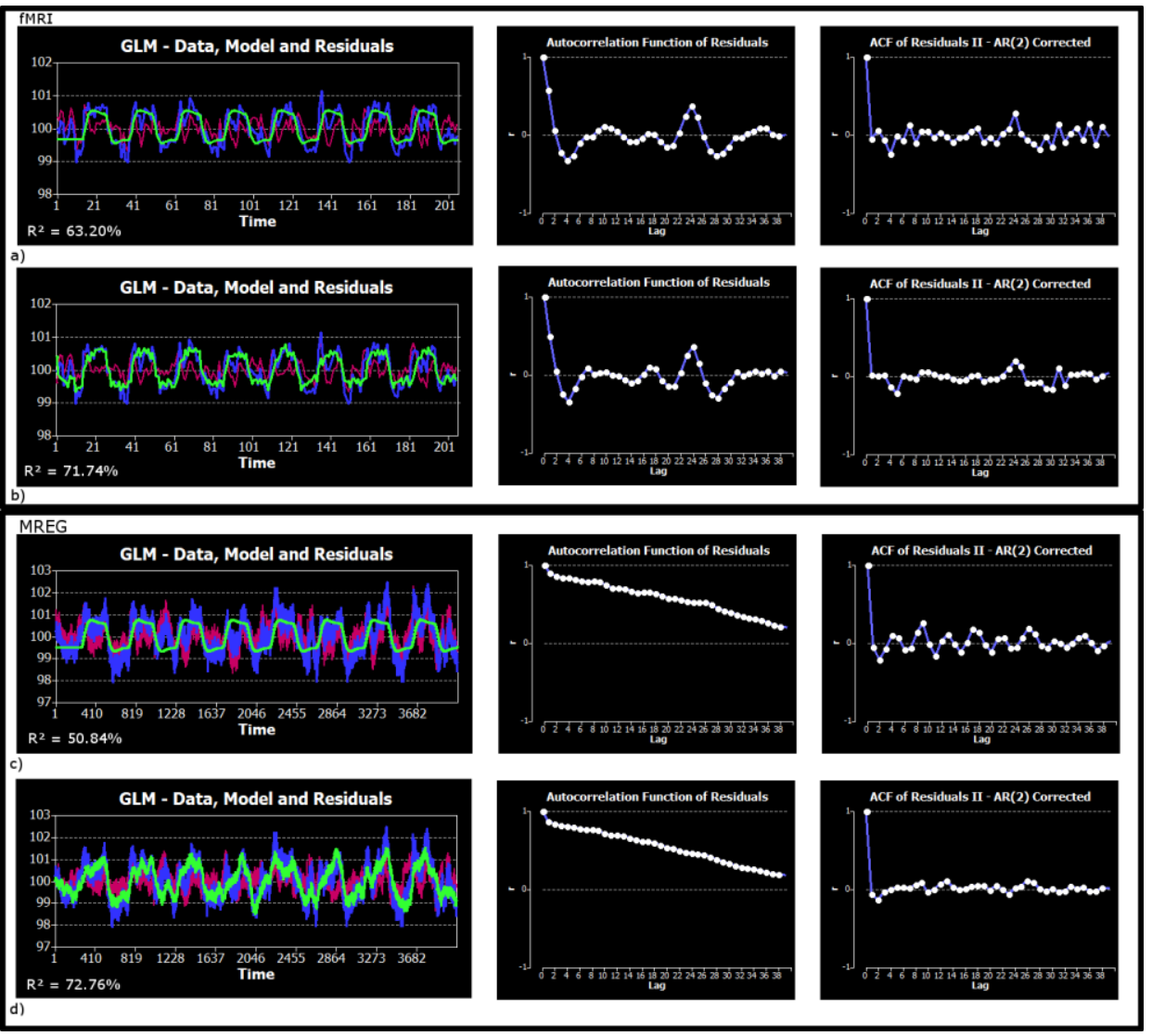

Figure 4: Time courses, models and residuals of an ROI GLM (P07, Motor Imagery). The left part of the figure shows the time course for SMS-EPI (top) and MREG (bottom) The upper plot ( $a$ and c) shows the GLM results without additional IC based confound predictors. The lower part ( $b$ and d) includes IC based confound predictors. The data is presented in blue, the model in green and in red the residuals (error) of the GLM. In the center the ACF for the residuals of the plot next to is shown. On the right the individual ACF's after $A R(2)$ correction are presented.

The explained variance of the GLM significantly increased $(p<0.001)$ for both the SMS-EPI and MREG sequence when ICA confound predictors were included. On average the explained variance increased from $26.91 \%$ to $48.42 \%$ for the SMS-EPI sequences and from $26.37 \%$ to $51.30 \%$ for MREG sequence. There was no significant difference between the increase of the SMS-EPI and MREG sequences. A detailed plot of all runs and the individual $R^{2}$ is presented in Figure 5. 

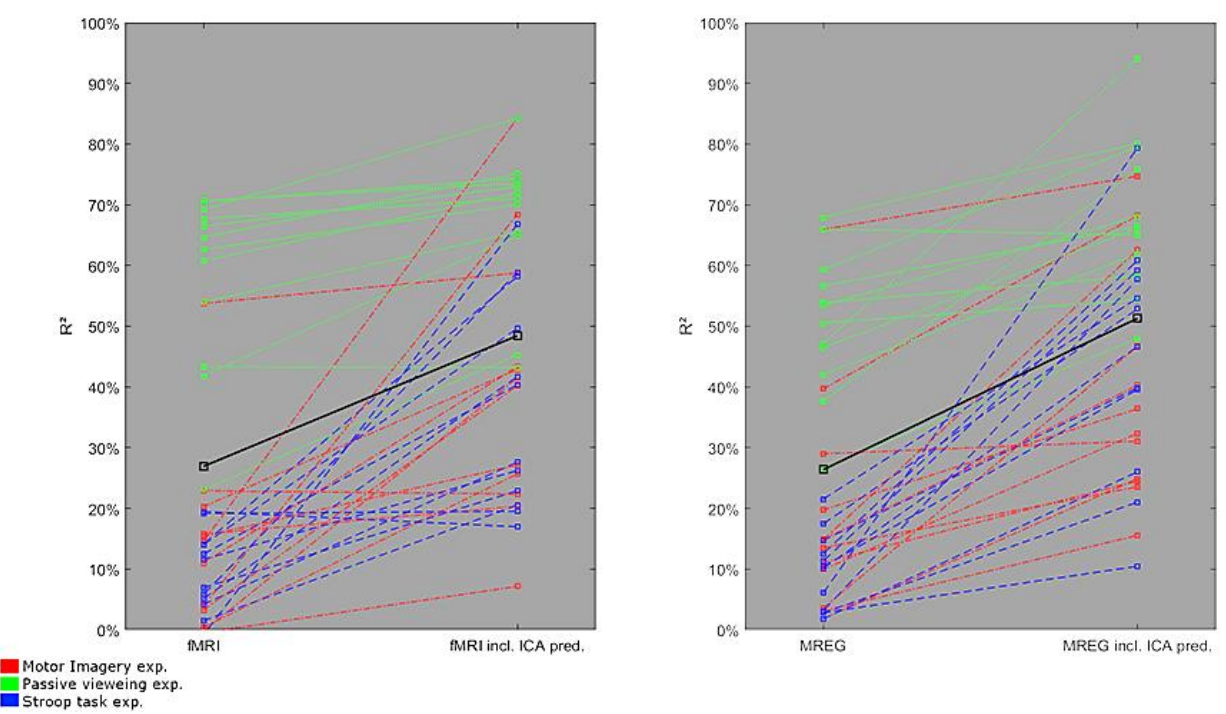

Figure 5: Explained variance of SMS-EPI and MREG ROI GLMs. In the left plot $R^{2}$ of the SMS-EPI GLM without additional confounds compared to the GLM including ICA confound predictors. On the right the same plot for MREG. Red being the motor imagery runs, green the passive viewing experiments and blue the response (Stroop) task sessions. Three ROI's were selected anatomically for each task.

\section{RFX analysis}

The RFX analysis revealed an overall consistency of SMS-EPI and MREG data. The comparison of the t-statistic of each map was based on Spearman RHO (Kornbrot, 2014). For the motor imagery run RHO was 0.4469 , for passive viewing 0.4762 and for the response (Stroop) task 0.4910 , all three of them were significant at $p<0.01$. An overview of the individual maps is shown in Figure 6. 


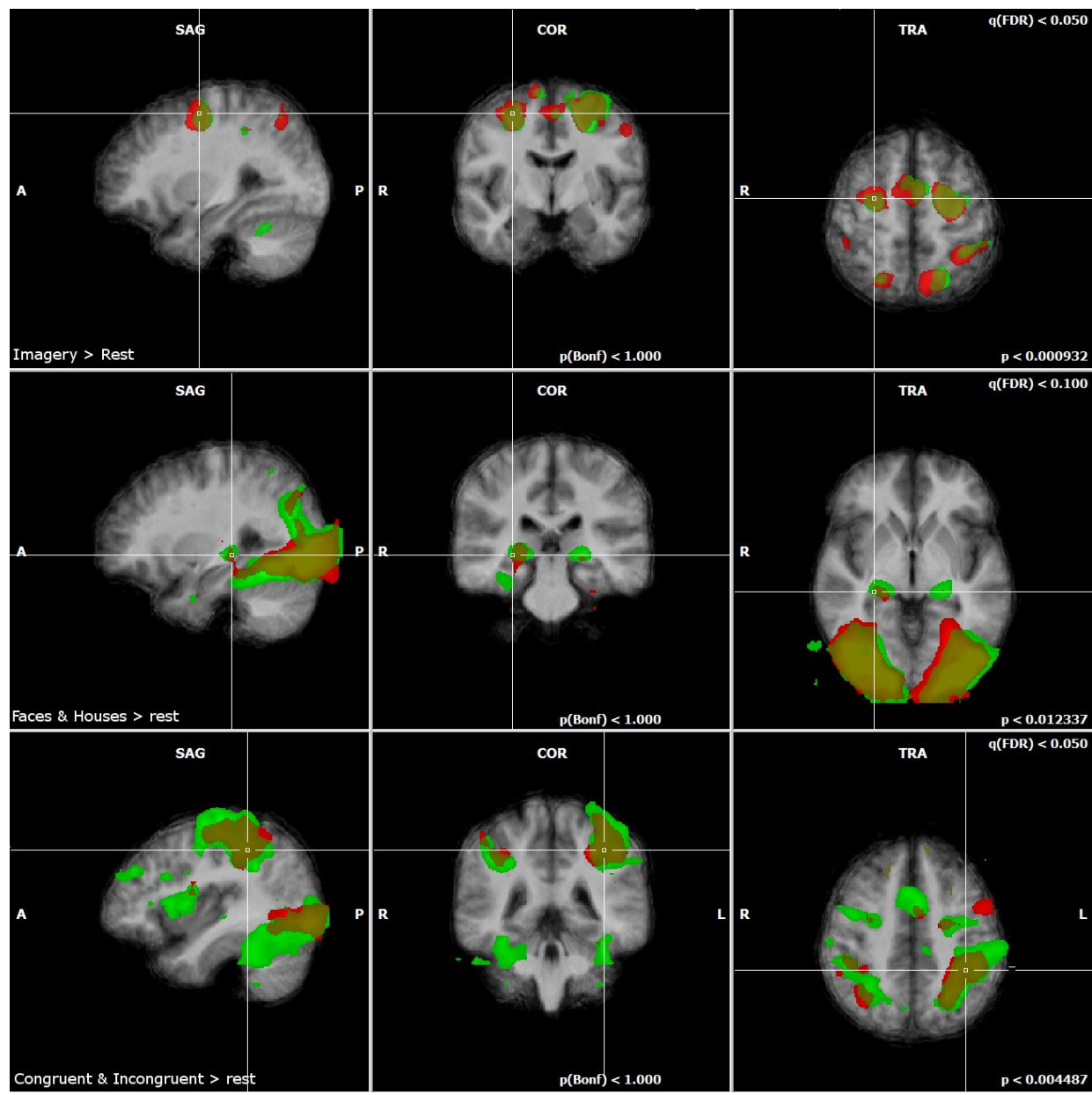

Figure 6: t-statistics of RFX GLM's comparing EPI-SMS (green) and MREG (red). Top part show RFX results for the motor imagery paradigm (imagery > rest). In the center the passive viewing experiment is shown (faces \& houses > rest) and in the bottom the Stroop task (congruent \& incongruent $>$ rest).

\section{tSNR}

The tSNR maps were compared using a pairwise t-test of the SMS-EPI and MREG runs (motor imagery, passive viewing, Stroop task) for each participant. The results are shown in Figure 7. Figure 7 left shows the SMS-EPI runs compared to the unfiltered MREG runs whereas Figure 7 right shows the filtered SMS-EPI runs compared to the filtered MREG runs using temporal smoothing with a gaussian kernel FWHM of $2 \mathrm{~s}$. 
Primary and supplementary motor areas as well as the ventrolateral prefrontal cortex, dorsal anterior cingulate and inferior temporal cortex show significantly higher tSNR for SMS-EPI compared to the unfiltered MREG runs ( $p<0.05$ Bonferroni corrected). After temporal filtering (Figure 7 right) the Primary and supplementary motor areas don't show any difference in tSNR, whereas the difference is maintained in parts of the dorsal anterior cingulate and the inferior temporal cortex.
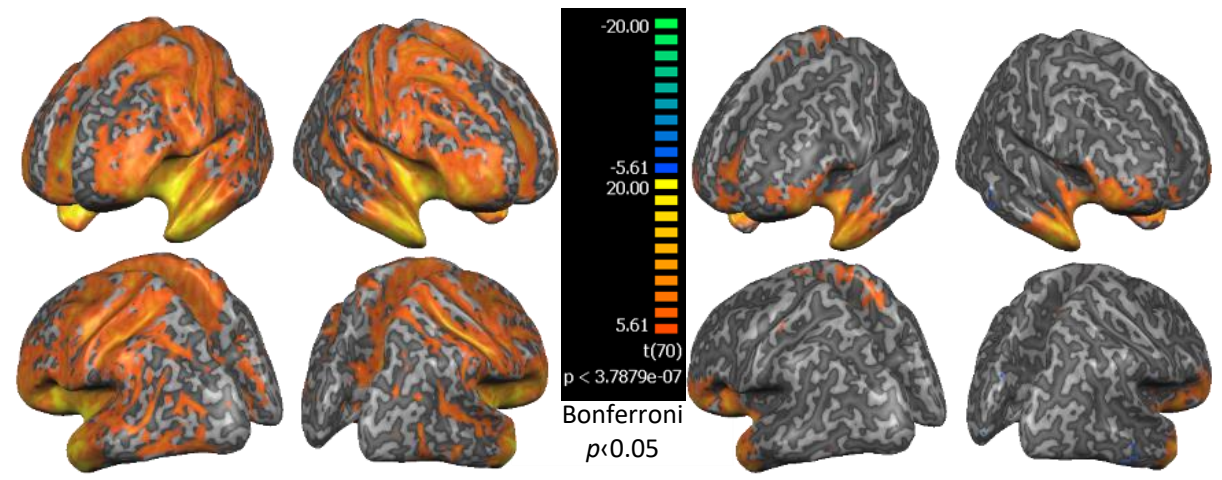

Figure 7: tSNR t-maps for EPI-SMS > MREG. The left side of the figure shows the t-test of EPI-SMS > MREG. On the right the same t-test is shown with an added temporal low pass filter for the MREG sequence. The maps are Bonferroni corrected ( $p_{\text {bonf }}<0.05$ ).

\section{Functional Connectivity}

The partial correlation of the generated white noise time courses convolved with the HRF (green time course in Figure 8) showed lower standard deviation for the high sampling rate (100ms sampling, blue time course) compared to the low temporal resolution ( $2 \mathrm{~s}$ sampling, red time course). The 2-s sampling was up-sampled to display the results in one graph. On the left side of Figure 8 a correlation window of 20s was used and on the right-hand side, a correlation window of $32 \mathrm{~s}$ was used. The blue curve (100-ms sampling) shows a lower standard deviation compared to the red curve ( $2 \mathrm{~s}$ sampling), which was significant at $p<0.01$. This finding was true for the short (10s) and long (20s) window (see left and right upper part of Figure 8). We also found this effect for the SMS-EPI (green curve) and MREG data (blue curve). High sampling MREG data showed significant lower standard deviation in the partial correlation $(p<0.01)$ for both for the short (10s) and long (20s) window (see $C$ ) and D) in Figure 8). 


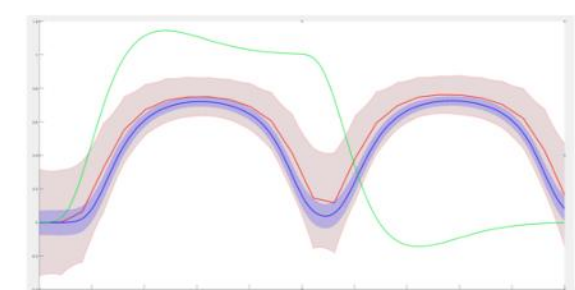

A)

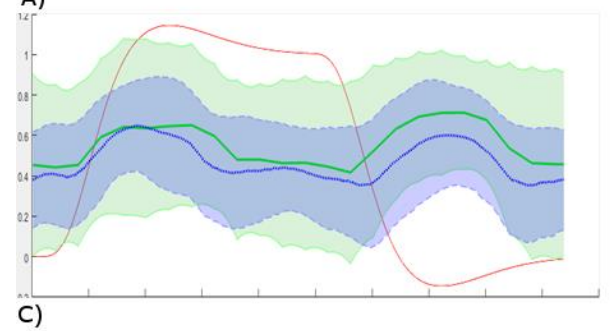

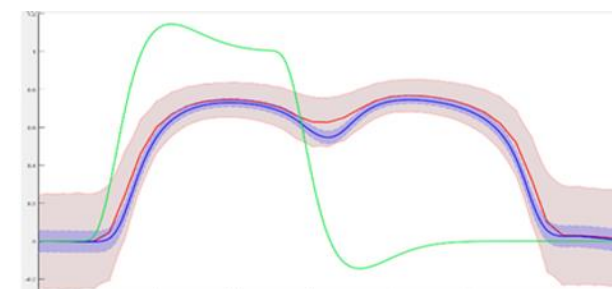

B)

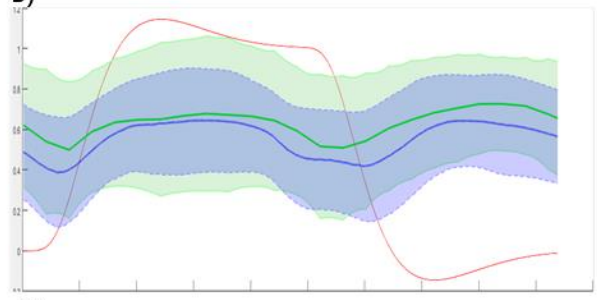

D)

Figure 8: Functional connectivity analysis using partial correlation. Top: simulated partial correlation for A) 10s moving window and B) $20 \mathrm{~s}$ moving window. Red curve, 2 s sampling, blue curve $100 \mathrm{~ms}$ sampling. Bottom: partial correlation of LSM and LPM (controlled by using a white matter ROI) in SMS-EPI (green curve) and MREG (blue curve) for C) $10 \mathrm{~s}$ and D) 20s. In red, as a reference, the standard double gamma HRF is depicted.

\section{Temporal down sampling of MREG to SMS-EPI resolution}

The down sampled MREG time course (to match the SMS-EPI temporal resolution) resulted in a significantly lower variance compared to the SMS-EPI $(p<0.05)$. As an example, a motor imagery trial with both time courses is shown in Figure 9. 


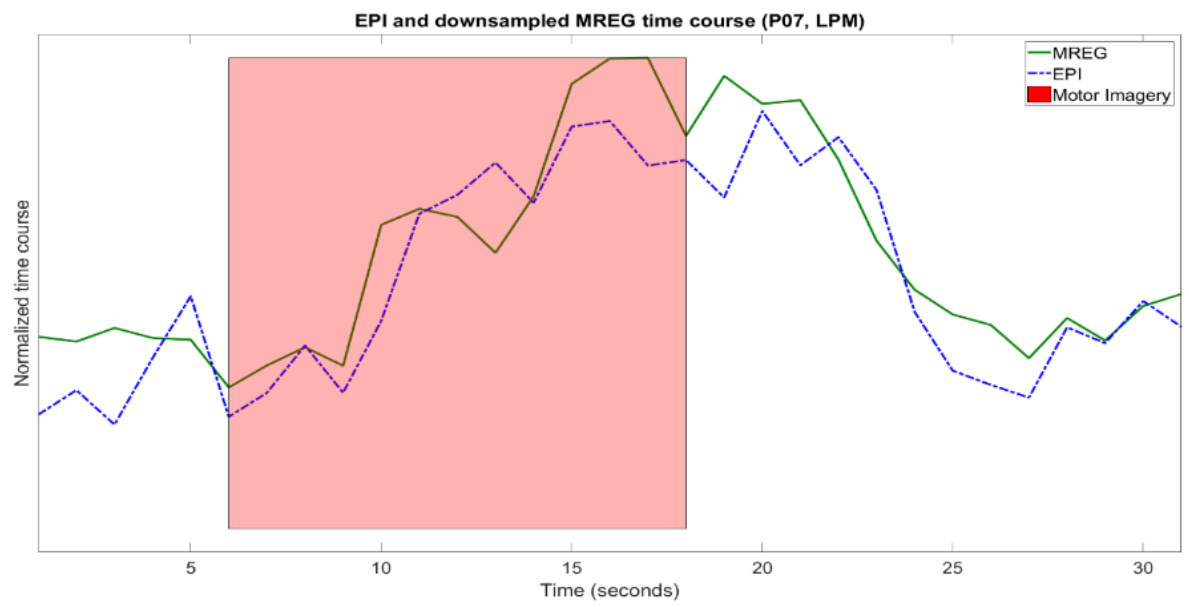

Figure 9: Down sampled MREG time course compared to the standard 2s TR EPI sequence. MREG time course has significant less variance compared to the SMS_EPI time course $(P<0.05)$. 


\section{DISCUSSION}

In this work we investigated the potential benefits of a recently developed parallel imaging technique MREG particularly for a possible use in real-time $\mathrm{BCl}$ and $\mathrm{NF}$ applications and compared it to a standard SMS-EPI sequence. Several parts of the analysis pipeline were investigated to point out specific differences in the pre- and postprocessing of the $\mathrm{fMRI}$ time-series generated by these sequences.

Generally, higher sampling rates (lower TRs) enable more detailed models of the HRF, e.g., for detecting the initial dip (Hu \& Yacoub, 2012) and to observe sub-second timing differences in task-based processes. However, higher sampling rates result in lower spatial resolution or brain coverage in non-parallel image acquisitions. Since for most NF applications the signal time course is used as a feedback source, the lower spatial resolution or coverage may be not necessarily a problem especially if the brain areas of interest can be targeted using a spatial resolution of $5-6 \mathrm{~mm}$ (or less).

Anyway, these drawbacks potentially resolve over time with the advent of parallel imaging techniques (Akin et al., 2017; Assländer et al., 2013; Hennig et al., 2007; Zahneisen et al., 2011, 2012).

MREG required adjustments to the standard fMRI processing pipeline, mainly, (1) functional/anatomical co-registration accounting for the lower spatial resolution of MREG, (2) adjusted statistical GLM analysis to correct for the higher temporal autocorrelation and (3) temporal filtering adaptations to remove heartbeat and respiration confounding signals. These differences will be discussed in the following sub-sections.

\section{MREG co-registration to anatomical data}

A short GRE sequence was used right before the MREG scan was collected and the time between both scans was set to $10 \mathrm{~s}$. It was assumed that during this period the head motion was absent or was very small between. To reduce potential motion in this interval and to keep its duration constant, we informed the participant before the start of the GRE sequence and did not communicate between GRE and MREG to keep the time low in which potential motion can occur. Even though this approach does not completely prohibit the potential of motionrelated misalignment, it seemed to be a good starting point and can be 
improved in following experiments by adding a (e.g., non-linear; (Klein et al., 2009) alignment step.

\section{ICA confound predictors}

To separate task-related signals from physiological fluctuations of no interest, ICA was used to identify noise components. The number of independent components is still a debate in current literature (Kairov et al., 2017). However, since the overall goal in this case was to only include confound predictors in the model, and not to find task relevant predictors or to spatially model functional networks, the question of the best number of ICA components is less relevant. Particularly, there were at least three reasons for keeping the number of ICA components very low: (i) the risk of losing physiological components is inversely related to the variance contribution of these components; thereby, the higher the contribution of physiological components, the better these will be extracted using relatively less temporal dimensions, whereas the lower the contribution of physiological components the lower the impact of potentially missing these components as confounds in the GLM; (ii) if two physiological components are not well separated (spatially) the removal of the (mixed) effects will operate as one, instead of two, confound predictors; (iii) the residual presence of task-related variance in a physiological component will be controlled by the increased correlation with the task predictor.

Apart from these qualitative expectations, we still checked for each participant that we found (at least) one heartbeat and one respiration component which were of most interest in this study. Indeed, the current results show that even when only using a few components, clear patterns for cardiac pulsation and respiration are quickly selected, as indicated in Figure 2. Interestingly, we were able to discern arterial structures like the circle of Willis when mapping these specific ICA confound components from the MREG data. As for the respiration component, a clear superficial pattern of the component map was visible. This is particularly encouraging for real-time $\mathrm{BCl}$ applications in which it is very important to control for respiration artifacts to ensure that participants did not achieve to reach a certain NF goal only by changing the respiration rate. Using MREG this could be implicitly controlled extracting the ICA component even without acquiring additional respiration information using a respiration belt. Nevertheless, 
further investigation is needed to further improve the selection of confound predictors especially for SMS-EPI and MREG studies using a very high sampling rate, and possibly build more abstract and general models to correct for physiological artifacts, eventually including spatial information. Such investigations will likely benefit from the use of several ICA component features (see, e. g., De Martino et al., 2007) that, besides the task correlation, have proven useful to separate BOLDrelated components from noise components, independently from the task activities.

\section{GLM analysis}

Because of the high temporal resolution, the parameters for the autocorrelation correction of the residuals were expected to be higher in MREG as compared to the standard SMS-EPI scanning parameters. The difference in temporal resolution of SMS-EPI and MREG data was the most important factor to consider in the GLM analysis, since their spatial properties did not differ notably. To ensure correct calculation of the $t$-values and avoid a statistical inflation caused by the high autocorrelation of the residuals in the MREG data, properties and assumptions of the GLM were investigated.

In $\mathrm{fMRI}$ data analysis with conventional TRs in the order of 1-3s the autocorrelation of the residuals can be corrected using low AR model orders (1-2; (Lenoski, Baxter, Karam, Maisog, \& Debbins, 2008), whereas for MREG we found that much higher model orders are necessary to correct for serial correlations. This is in line with recent reports on fast sampling fMRI experiments (Bollmann, Puckett, Cunnington, \& Barth, 2018; Corbin, Todd, Friston, \& Callaghan, 2018). We as well observed that a higher explained variance (e.g., by adding physiological confound predictors to the model) was linked to a lower estimated AR orders, which reflected a lower autocorrelation in the residuals. This is an important difference compared to sequences using a low TR and it is crucial to fully take advantage of the high temporal resolution of MREG sequence when modeling the expected time course in the design matrix by including specific confound predictors for cardiac pulsation and respiration related signals. Even though the inclusion of ICA component time courses as model predictors is in some cases difficult, the overall effect of improving the explained variance is expectedly the same with 
respect to including externally measured pulse and respiration data because of the high correlation of these time courses.

Even if the explained variance in both sequences is highly comparable, MREG is more sensitive to capture heartbeat and respiration information suggests that this sequence is far more sensitive than conventional $\mathrm{fMRI}$ where the heartbeat information can not directly be separated from the signal because of the low temporal resolution. While these effects are clearly visible in the single-subject analyses, the differences in the RFX group results between SMS-EPI and MREG sequences are less straightforward to explain or display. In fact, they can be caused by both the different sensitivity of the two sequences and by individual subject performance variability. All in all, more studies are necessary to clarify this matter.

In standard $\mathrm{fMRI}$ analysis pipelines, the detection of motion remains a problem which needs further investigation (Leonard, Flournoy, Lewis-de los Angeles, \& Whitaker, 2017; Todd, Josephs, Callaghan, Lutti, \& Weiskopf, 2015; Yakupov, Lei, Hoffmann, \& Speck, 2017). Two different correction methods, retrospective and prospective, are most often used in fMRI. Prospective motion correction shows better results in correction of motion artifacts, especially in the intra volume motion, which cannot be corrected using standard retrospective correction methods (Todd et al., 2015). For MREG the intra slice and volume motion correction problem is intrinsically reduced by the higher temporal resolution allowed by the acquisition method.

\section{The impact on real-time $\mathrm{BCl} /$ neurofeedback experiments}

Due to more and more attention to applications of real-time neuroimaging methods, specifically in the field of fMRI (Sulzer et al., 2013; Thibault, MacPherson, Lifshitz, Roth, \& Raz, 2018), the implementation of a higher temporal resolution is a very important goal. We showed that physiological components are clearly visible in the MREG data and exhibited that they can be extracted to correct the measured brain signal in real-time. This allows to control for brainmodulation effects caused by changes in cardiac pulsation or respiration. This is especially important to ensure that possible improvements in behavior by using $\mathrm{BCl} / \mathrm{NF}$ setups are not just caused by learning to regulate breathing but due to a real cognitive control. 


\section{CONCLUSION}

In this manuscript we investigated the different impact of a standard SMS-EPI sequence and the more recently developed MREG sequence on high temporal resolution fMRI. For both sequences the same coverage and spatial resolution was used. The temporal resolution of the MREG sequence was, however, 20 times higher than the SMS-EPI sequence using a conventional TR of $2 \mathrm{~s}$ as typically used in (real-time) fMRI recordings. Overall the results of SMS-EPI and MREG data analysis were comparable even though different preprocessing and analysis techniques needed to be adapted for the MREG compared to standard SMS-EPI data processing. Most importantly, a much higher AR model order of $\sim 20$ was required to correct for serial correlations in the GLM residuals. Thereby, the higher temporal resolution of MREG allows disentangling explicitly physiological influences from cardiac pulsation and respiration using ICA and therefore has the potential to be more sensitive compared to standard SMS-EPI.

tSNR was also comparable between sequences, although known sequence-specific geometric image distortions were observed. Further research is necessary to create more detailed models for sequences using such a high temporal resolution to explain more variance which, as we showed, lowers the temporal autocorrelation in the residuals and increases the power of the statistical analysis.

This exploratory research serves a more comprehensive utilization of the findings for following rt-MREG based NF/BCl research. 


\section{ACKNOWLEDGMENTS}

This research was financially supported by the European Commission's Health Cooperation Work Programme of the 7th Framework Programme, under the Grant Agreement $n^{\circ} 602450$ (IMAGEMEND) and $n^{\circ} 602186$ (BRAINTRAIN). Scanning hours were provided by Scannexus (Maastricht, Netherlands). BAP is funded by the Netherland's Organisation for Scientific Research (NWO 016.Vidi.178.052) and the National Institute of Health (R01MH111444, PI Feinberg).

This article reflects only the authors' view and the funding sources are not liable for any use that may be made of the information contained therein. 


\section{References}

Akin, B., Lee, H. L., Hennig, J., \& LeVan, P. (2017). Enhanced subject-specific resting-state network detection and extraction with fast fMRI. Human Brain Mapping, 38(2), 817-830. https://doi.org/10.1002/hbm.23420

Assländer, J., Zahneisen, B., Hugger, T., Reisert, M., Lee, H. L., LeVan, P., \& Hennig, J. (2013). Single shot whole brain imaging using spherical stack of spirals trajectories. Neurolmage, 73, 59-70. https://doi.org/10.1016/j.neuroimage.2013.01.065

Barker, J. W., Aarabi, A., \& Huppert, T. J. (2013). Autoregressive model based algorithm for correcting motion and serially correlated errors in fNIRS. Biomedical Optics $\quad$ Express, 1366. https://doi.org/10.1364/BOE.4.001366

Barker, J. W., Rosso, A. L., Sparto, P. J., \& Huppert, T. J. (2016). Correction of motion artifacts and serial correlations for real-time functional nearinfrared spectroscopy. Neurophotonics, 3(3), 031410. https://doi.org/10.1117/1.NPh.3.3.031410

Bastos, A. M., \& Schoffelen, J.-M. (2016). A Tutorial Review of Functional Connectivity Analysis Methods and Their Interpretational Pitfalls. Frontiers in Systems Neuroscience, 9, 175. https://doi.org/10.3389/fnsys.2015.00175

Bollmann, S., Puckett, A. M., Cunnington, R., \& Barth, M. (2018). Serial correlations in single-subject fMRI with sub-second TR. Neurolmage, 166, 152-166. https://doi.org/10.1016/j.neuroimage.2017.10.043

Boynton, G. M., Engel, S. A., Glover, G. H., \& Heeger, D. J. (1996). Linear systems analysis of functional magnetic resonance imaging in human V1. Journal of Neuroscience, 16(13), 4207-21. https://doi.org/10.1523/JNEUROSCI.16-13-04207.1996

Burg, J. P. (1975). Maximum Entropy Spectral Analysis. Astronomy and Astrophysics Supplement, Vol. 15, p.383. Retrieved from http://labs.adsabs.harvard.edu/adsabs/abs/1974A\&AS...15..383A/

Canuet, L. (2015). Neurorehabilitation in Stroke: The Role of Functional Connectivity. International Journal of Neurorehabilitation, 02(03), 1-2. https://doi.org/10.4172/2376-0281.1000172

Corbin, N., Todd, N., Friston, K. J., \& Callaghan, M. F. (2018). Accurate modeling of temporal correlations in rapidly sampled $\mathrm{fMRI}$ time series. Human Brain Mapping. https://doi.org/10.1002/hbm.24218

De Hoon, M. J. L., Van Der Hagen, T. H. J. J., Schoonewelle, H., \& Van Dam, H. (1996). Why Yule-Walker should not be used for autoregressive modelling. Annals of Nuclear Energy, 23(15), 1219-1228. https://doi.org/10.1016/0306-4549(95)00126-3

De Martino, F., Gentile, F., Esposito, F., Balsi, M., Di Salle, F., Goebel, R., \& 
Formisano, E. (2007). Classification of fMRI independent components using IC-fingerprints and support vector machine classifiers. Neurolmage, 34(1), 177-194. https://doi.org/10.1016/j.neuroimage.2006.08.041

Díez-Cirarda, M., Ojeda, N., Peña, J., Cabrera-Zubizarreta, A., Lucas-Jiménez, O., Gómez-Esteban, J. C., ... Ibarretxe-Bilbao, N. (2017). Increased brain connectivity and activation after cognitive rehabilitation in Parkinson's disease: a randomized controlled trial. Brain Imaging and Behavior, 11(6), 1640-1651. https://doi.org/10.1007/s11682-016-9639-x

Eklund, A., Friman, O., Andersson, M., \& Knutsson, H. (2011). Comparing fMRI Activity Maps from GLM and CCA at the Same Significance Level by Fast Random Permutation Tests on the GPU. Retrieved from http://www.divaportal.org/smash/record.jsf?pid=diva2:402372

Fadili, M. J., Ruan, S., Bloyet, D., \& Mazoyer, B. (2003). Analysis of fMRI time series. Human Brain Function, 178(1), 160-178. https://doi.org/10.1006/nimg.1995.1007

Feinberg, D. A., Moeller, S., Smith, S. M., Auerbach, E., Ramanna, S., Glasser, M. F., ... Yacoub, E. (2010). Multiplexed echo planar imaging for subsecond whole brain fmri and fast diffusion imaging. PLOS ONE, 5(12), e15710. https://doi.org/10.1371/journal.pone.0015710

Feinberg, D. A., \& Setsompop, K. (2013). Ultra-fast MRI of the human brain with simultaneous multi-slice imaging. Journal of Magnetic Resonance, 229, 90-100. https://doi.org/10.1016/j.jmr.2013.02.002

Feinberg, D. A., \& Yacoub, E. (2012). The rapid development of high speed, resolution and precision in fMRI. Neurolmage, 62(2), 720-725. https://doi.org/10.1016/j.neuroimage.2012.01.049

Friman, O., \& Westin, C. F. (2005). Resampling fMRI time series. Neurolmage, 25(3), 859-867. https://doi.org/10.1016/j.neuroimage.2004.11.046

Friston, K. J. (2011). Functional and Effective Connectivity: A Review. Brain Connectivity, 1(1), 13-36. https://doi.org/10.1089/brain.2011.0008

Friston, K. J., Josephs, O., Zarahn, E., Holmes, A. P., Rouquette, S., \& Poline, J. B. (2000). To smooth or not to smooth? Bias and efficiency in fMRI timeseries analysis. Neurolmage, 12(2), 196-208. https://doi.org/10.1006/nimg.2000.0609

Glover, G. H. (1999). Deconvolution of impulse response in event-related BOLD fMRI. Neurolmage, 9(4), 416-429.

https://doi.org/10.1006/nimg.1998.0419

Greve, D. N., \& Fischl, B. (2009). Accurate and robust brain image alignment using boundary-based registration. Neurolmage, 48(1), 63-72. https://doi.org/10.1016/j.neuroimage.2009.06.060

Hao, Y., Khoo, H. M., von Ellenrieder, N., \& Gotman, J. (2017). Subject-level reliability analysis of fast fMRI with application to epilepsy. Magnetic Resonance in Medicine, 78(1), 370-382. 
https://doi.org/10.1002/mrm.26365

Hennig, J., Zhong, K., \& Speck, O. (2007). MR-Encephalography: Fast multichannel monitoring of brain physiology with magnetic resonance. Neurolmage, 34(1),

212-219.

https://doi.org/10.1016/j.neuroimage.2006.08.036

Holmes, C. J., Hoge, R., Collins, L., Woods, R., Toga, A. W., \& Evans, A. C. (1998). Enhancement of MR images using registration for signal averaging. Journal of Computer Assisted Tomography, 22(2), 324-333. https://doi.org/10.1097/00004728-199803000-00032

Hsu, Y. C., Chu, Y. H., Tsai, S. Y., Kuo, W. J., Chang, C. Y., \& Lin, F. H. (2017). Simultaneous multi-slice inverse imaging of the human brain. Scientific Reports, 7(1), 17019. https://doi.org/10.1038/s41598-017-16976-0

$\mathrm{Hu}, \mathrm{X} .$, \& Yacoub, E. (2012). The story of the initial dip in fMRI. Neurolmage, 62(2), 1103-1108. https://doi.org/10.1016/j.neuroimage.2012.03.005

Hutchison, R. M., Womelsdorf, T., Allen, E. A., Bandettini, P. A., Calhoun, V. D., Corbetta, M., ... Chang, C. (2013). Dynamic functional connectivity: Promise, issues, and interpretations. Neurolmage, 80, 360-378. https://doi.org/10.1016/j.neuroimage.2013.05.079

Hyvärinen, A. (1999). Fast and robust fixed-point algorithms for independent component analysis. IEEE Transactions on Neural Networks, 10(3), 626634. https://doi.org/10.1109/72.761722

Kairov, U., Cantini, L., Greco, A., Molkenov, A., Czerwinska, U., Barillot, E., \& Zinovyev, A. (2017). Determining the optimal number of independent components for reproducible transcriptomic data analysis. $B M C$ Genomics, 18(1), 712. https://doi.org/10.1186/s12864-017-4112-9

Kanwisher, N. G., McDermott, J., \& Chun, M. M. (1997). The fusiform face area: A module in human extrastriate cortex specialized for face perception. Journal of Neuroscience, 17(11), 4302-4311. https://doi.org/10.1523/JNEUROSCI.17-11-04302.1997

Karahanoğlu, F. I., \& Van De Ville, D. (2017). Dynamics of Large-Scale fMRI Networks: Deconstruct Brain Activity to Build Better Models of Brain Function. Current Opinion in Biomedical Engineering, 3, 28-36. https://doi.org/10.1016/j.cobme.2017.09.008

Kay, S. M., \& Marple, S. L. (1981). Spectrum Analysis-A Modern Perspective. Proceedings of the IEEE, 69(11), 1380-1419. https://doi.org/10.1109/PROC.1981.12184

Klein, A., Andersson, J., Ardekani, B. A., Ashburner, J., Avants, B., Chiang, M.-C., ... Parsey, R. V. (2009). Evaluation of 14 nonlinear deformation algorithms applied to human brain MRI registration. Neurolmage, 46(3), 786-802. https://doi.org/10.1016/j.neuroimage.2008.12.037

Kornbrot, D. (2014). Spearman's Rho. In Wiley StatsRef: Statistics Reference Online. Chichester, UK: John Wiley \& Sons, Ltd. 
https://doi.org/10.1002/9781118445112.stat06541

Krause, F., Benjamins, C., Lührs, M., Eck, J., Noirhomme, Q., Rosenke, M., ... Goebel, R. (2017). Real-time fMRI-based self-regulation of brain activation across different visual feedback presentations. Brain-Computer Interfaces, $4(1-2)$,

87-101. https://doi.org/10.1080/2326263X.2017.1307096

Lee, H. L., Zahneisen, B., Hugger, T., LeVan, P., \& Hennig, J. (2013). Tracking dynamic resting-state networks at higher frequencies using MRencephalography. Neurolmage, 216-222. https://doi.org/10.1016/j.neuroimage.2012.10.015

Lenoski, B., Baxter, L. C., Karam, L. J., Maisog, J., \& Debbins, J. (2008). On the performance of autocorrelation estimation algorithms for $\mathrm{fMRI}$ analysis. IEEE Journal on Selected Topics in Signal Processing, 2(6), 828-838. https://doi.org/10.1109/JSTSP.2008.2007819

Leonard, J., Flournoy, J., Lewis-de los Angeles, C. P., \& Whitaker, K. (2017). How much motion is too much motion? Determining motion thresholds by sample size for reproducibility in developmental resting-state MRI. Research Ideas and Outcomes, 3, e12569. https://doi.org/10.3897/rio.3.e12569

Lewis, L. D., Setsompop, K., Rosen, B. R., \& Polimeni, J. R. (2016). Fast fMRI can detect oscillatory neural activity in humans. Proceedings of the National Academy of Sciences, 113(43), E6679-E6685. https://doi.org/10.1073/pnas.1608117113

LI, S.-M. (2014). Burmanesque mini review. Adv. Synth. Catal., 201, 1-6. https://doi.org/10.1002/adsc.201

Lin, F. H., Tsai, K. W. K., Chu, Y. H., Witzel, T., Nummenmaa, A., Raij, T., ... Belliveau, J. W. (2012). Ultrafast inverse imaging techniques for fMRI. Neurolmage, 62(2), 699-705. https://doi.org/10.1016/j.neuroimage.2012.01.072

Locascio, J. J., Jennings, P. J., Moore, C. I., \& Corkin, S. (1997). Time series analysis in the time domain and resampling methods for studies of functional magnetic resonance brain imaging. Human Brain Mapping, 5(3), 168-193. $\quad$ https://doi.org/10.1002/(SICI)10970193(1997)5:3<168::AID-HBM3>3.0.CO;2-1

Lührs, M., Sorger, B., Goebel, R., \& Esposito, F. (2017). Automated selection of brain regions for real-time fMRI brain-computer interfaces. Journal of Neural Engineering, 14(1), 016004. https://doi.org/10.1088/17412560/14/1/016004

McKeown, M. J. (2000). Detection of consistently task-related activations in fMRI data with hybrid independent component analysis. Neurolmage, 11(1), 24-35. https://doi.org/10.1006/nimg.1999.0518

McKeown, M. J., Hansen, L. K., \& Sejnowsk, T. J. (2003). Independent 
component analysis of functional MRI: What is signal and what is noise? Current Opinion in Neurobiology, 13(5), 620-629. https://doi.org/10.1016/j.conb.2003.09.012

McKeown, M. J., Makeig, S., Brown, G. G., Jung, T. P., Kindermann, S. S., Bell, A. J., \& Sejnowski, T. J. (1998). Analysis of $\mathrm{fMRI}$ data by blind separation into independent spatial components. Human Brain Mapping, 6(3), 160-188. https://doi.org/10.1002/(SICI)1097-0193(1998)6:3<160::AIDHBM5>3.0.CO;2-1

Moeller, S., Yacoub, E., Olman, C. A., Auerbach, E., Strupp, J., Harel, N., \& Uğurbil, K. (2010). Multiband multislice GE-EPI at 7 tesla, with 16-fold acceleration using partial parallel imaging with application to high spatial and temporal whole-brain FMRI. Magnetic Resonance in Medicine, 63(5), 1144-1153. https://doi.org/10.1002/mrm.22361

Monti, M. (2011). Statistical Analysis of fMRI Time-Series: A Critical Review of the GLM Approach. Frontiers in Human Neuroscience, 5, 28. https://doi.org/10.3389/fnhum.2011.00028

Murphy, K., Bodurka, J., \& Bandettini, P. A. (2007). How long to scan? The relationship between $\mathrm{fMRI}$ temporal signal to noise ratio and necessary scan duration. Neurolmage, 34(2), 565-574. https://doi.org/10.1016/j.neuroimage.2006.09.032

Ochmann, S., Dyrba, M., Grothe, M. J., Kasper, E., Webel, S., Hauenstein, K., \& Teipel, S. J. (2017). Does Functional Connectivity Provide a Marker for Cognitive Rehabilitation Effects in Alzheimer's Disease? An Interventional Study. Journal of Alzheimer's Disease, 57(4), 1303-1313. https://doi.org/10.3233/JAD-160773

Peterson, B. S., Skudlarski, P., Gatenby, J. C., Zhang, H., Anderson, A. W., \& Gore, J. C. (1999). An fMRI study of stroop word-color interference: Evidence for cingulate subregions subserving multiple distributed attentional systems. Biological Psychiatry, 45(10), 1237-1258. https://doi.org/10.1016/S0006-3223(99)00056-6

Pfeuffer, J., Van Moortele, P. F. De, Ugurbil, K., Hu, X., \& Glover, G. H. (2002). Correction of physiologically induced global off-resonance effects in dynamic echo-planar and spiral functional imaging. Magnetic Resonance in Medicine, 47(2), 344-353. https://doi.org/10.1002/mrm.10065

Pollock, D. S. G., \& Green, R. C. (1999). Handbook of Time Series Analysis, Signal Processing, and Dynamics. Handbook of Time Series Analysis, Signal Processing, and Dynamics. https://doi.org/10.1016/B978012560990-6/50000-2

Poser, B. A., \& Setsompop, K. (2018). Pulse sequences and parallel imaging for high spatiotemporal resolution MRI at ultra-high field. Neurolmage, 168, 101-118. https://doi.org/10.1016/J.NEUROIMAGE.2017.04.006

Posse, S., Ackley, E., Mutihac, R., Zhang, T., Hummatov, R., Akhtari, M., ... 
Yonas, H. (2013). High-Speed Real-Time Resting-State fMRI Using MultiSlab Echo-Volumar Imaging. Frontiers in Human Neuroscience, 7, 479. https://doi.org/10.3389/fnhum.2013.00479

Rabrait, C., Ciuciu, P., Ribés, A., Poupon, C., Le Roux, P., Dehaine-Lambertz, G., ... Lethimonnier, F. (2008). High temporal resolution functional MRI using parallel echo volumar imaging. Journal of Magnetic Resonance Imaging, 27(4), 744-753. https://doi.org/10.1002/jmri.21329

Riemenschneider, B., Levan, P., Reisert, M., \& Hennig, J. (2015). Nonlinear trajectories in real-time fMRI using target volumes. Abstract \#2055, 4(7), 232908. Retrieved from https://dev.ismrm.org/2015/2055.html

Rogers, B. P., Katwal, S. B., Morgan, V. L., Asplund, C. L., \& Gore, J. C. (2010). Functional MRI and multivariate autoregressive models. Magnetic Resonance Imaging, 28(8), 1058-1065. https://doi.org/10.1016/j.mri.2010.03.002

Sahib, A. K., Erb, M., Marquetand, J., Martin, P., Elshahabi, A., Klamer, S., ... Focke, N. K. (2018). Evaluating the impact of fast-fMRI on dynamic functional connectivity in an event-based paradigm. PLOS ONE, 13(1), e0190480. https://doi.org/10.1371/journal.pone.0190480

Setsompop, K., Gagoski, B. A., Polimeni, J. R., Witzel, T., Wedeen, V. J., \& Wald, L. L. (2012). Blipped-controlled aliasing in parallel imaging for simultaneous multislice echo planar imaging with reduced g-factor penalty. Magnetic Resonance in Medicine, 67(5), 1210-1224. https://doi.org/10.1002/mrm.23097

Sorger, B., Reithler, J., Dahmen, B., \& Goebel, R. (2012). A real-time fMRI-based spelling device immediately enabling robust motor-independent communication. Current Biology, 22(14), 1333-1338. https://doi.org/10.1016/j.cub.2012.05.022

Sulzer, J., Haller, S., Scharnowski, F., Weiskopf, N., Birbaumer, N., Blefari, M. L., ... Sitaram, R. (2013). Real-time fMRI neurofeedback: Progress and challenges. Neurolmage, 76, 386-399. https://doi.org/10.1016/j.neuroimage.2013.03.033

Tak, S., \& Ye, J. C. (2014). Statistical analysis of fNIRS data: A comprehensive review. Neurolmage, 85 , 72-91. https://doi.org/10.1016/j.neuroimage.2013.06.016

Thibault, R. T., MacPherson, A., Lifshitz, M., Roth, R. R., \& Raz, A. Neurofeedback with fMRI: A critical systematic review, 172 Neurolmage $\S$ (2018). Academic

Press. https://doi.org/10.1016/j.neuroimage.2017.12.071

Todd, N., Josephs, O., Callaghan, M. F., Lutti, A., \& Weiskopf, N. (2015). Prospective motion correction of $3 \mathrm{D}$ echo-planar imaging data for functional MRI using optical tracking. Neurolmage, 113, 1-12. https://doi.org/10.1016/j.neuroimage.2015.03.013 
Uga, M., Dan, I., Sano, T., Dan, H., \& Watanabe, E. (2014). Optimizing the general linear model for functional near-infrared spectroscopy: an adaptive hemodynamic response function approach. Neurophotonics, 1(1), 015004. https://doi.org/10.1117/1.NPh.1.1.015004

Welvaert, M., \& Rosseel, Y. (2013). On the definition of signal-to-noise ratio and contrast-to-noise ratio for fMRI data. PLOS ONE, 8(11), e77089. https://doi.org/10.1371/journal.pone.0077089

Yakupov, R., Lei, J., Hoffmann, M. B., \& Speck, O. (2017). False fMRI activation after motion correction. Human Brain Mapping, 38(9), 4497-4510. https://doi.org/10.1002/hbm.23677

Zahneisen, B., Grotz, T., Lee, K. J., Ohlendorf, S., Reisert, M., Zaitsev, M., \& Hennig, J. (2011). Three-dimensional MR-encephalography: Fast volumetric brain imaging using rosette trajectories. Magnetic Resonance in Medicine, 65(5), 1260-1268. https://doi.org/10.1002/mrm.22711

Zahneisen, B., Hugger, T., Lee, K. J., Levan, P., Reisert, M., Lee, H. L., ... Hennig, J. (2012). Single shot concentric shells trajectories for ultra fast fMRI. Magnetic Resonance in Medicine, 68(2), 484-494. https://doi.org/10.1002/mrm.23256

Zilverstand, A., Sorger, B., Zimmermann, J., Kaas, A., \& Goebel, R. (2014). Windowed correlation: A suitable tool for providing dynamic fMRI-based functional connectivity neurofeedback on task difficulty. PLOS ONE, 9(1), e85929. https://doi.org/10.1371/journal.pone.0085929 


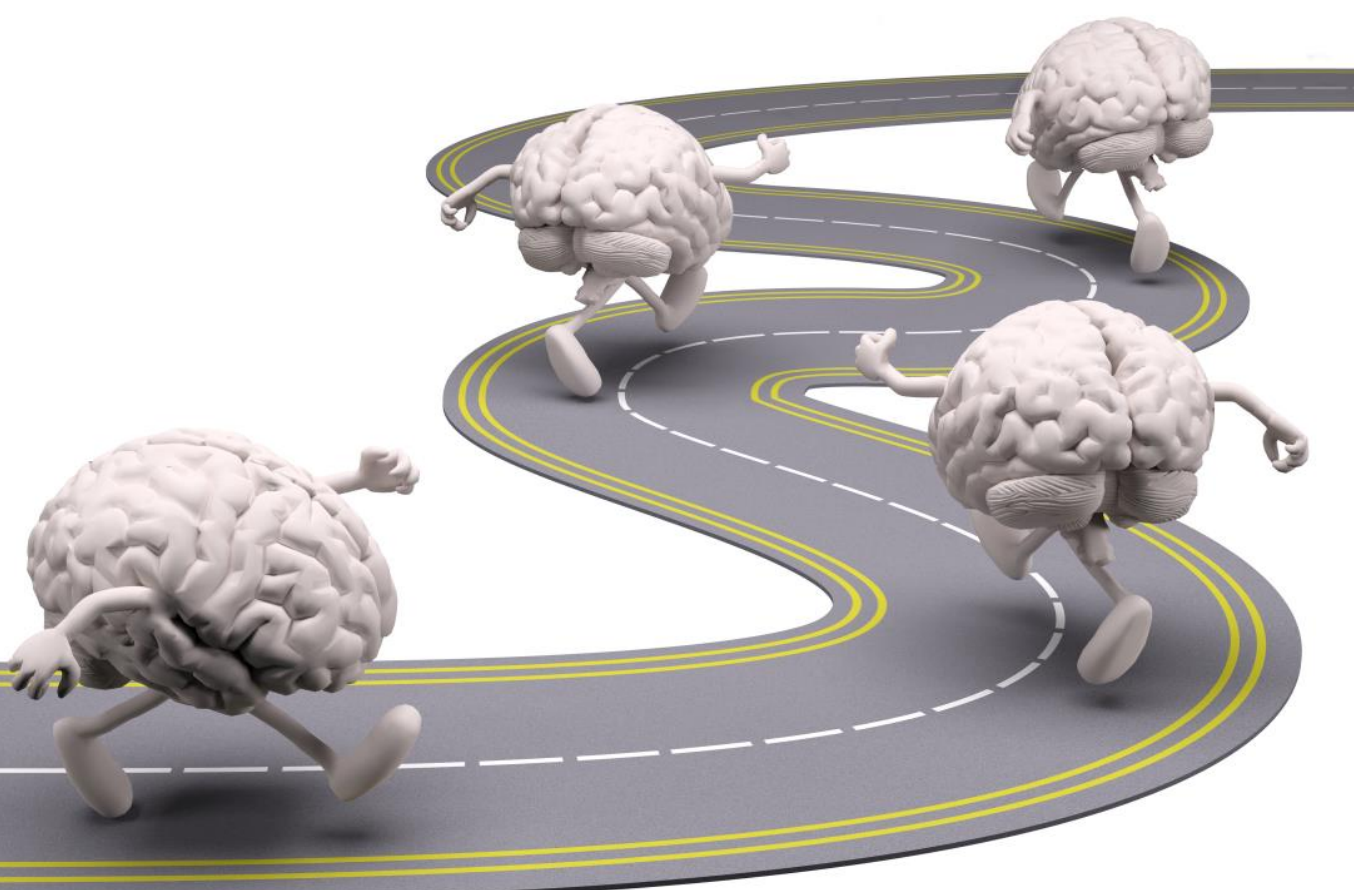




\section{Chapter 4}

\section{Turbo-Satori - A neurofeedback and BCl toolbox for real-time fNIRS}

Corresponding publication:

Lührs, M., Goebel, R., 2017. Turbo-Satori: a neurofeedback and brain-computer interface toolbox for real-time functional near-infrared spectroscopy. Neurophotonics, 4(4), 041504. 


\section{ABSTRACT}

Turbo-Satori is a neurofeedback and $\mathrm{BCl}$ toolbox for real-time functional near-infrared spectroscopy (fNIRS). It incorporates multiple pipelines from real-time preprocessing and analysis to neurofeedback and $\mathrm{BCl}$ applications. The toolbox is designed with a focus in usability, enabling a fast setup and execution of real-time experiments. Turbo-Satori uses an incremental recursive least-squares procedure for real-time general linear model calculation (rlsGLM) and support vector machine (SVM) classifiers for advanced $\mathrm{BCl}$ applications. It communicates directly with common NIRx fNIRS hardware and was tested extensively ensuring that the calculations can be performed in real-time without a significant change in calculation times for all sampling intervals during ongoing experiments of up to six hours of recording. Enabling immediate access to advanced processing features allows the use of this toolbox also for students and non-experts in the field of fNIRS data acquisition and processing. Flexible network interfaces allow 3rd party stimulus applications to access the processed data and calculated statistics in real-time so that this information can be easily incorporated in neurofeedback or $\mathrm{BCl}$ presentations. 


\section{INTRODUCTION}

In recent years, the developments of brain computer interfaces (BCls) based on functional near infrared spectroscopy (fNIRS) have been continuously evolved establishing its use for human computer interaction ( $\mathrm{HCl}$ )(Girouard, 2010; Lorenz, Pascual, Blankertz, \& Vidaurre, 2014; Solovey, 2012) as well as for brain state decoding(Lührs, Sorger, Goebel, \& Esposito, 2017; Naci, Cusack, Jia, \& Owen, 2013; Sato et al., 2013; Sorger et al., 2009; Sorger, Reithler, Dahmen, \& Goebel, 2012) and neurorehabilitation(Mihara \& Miyai, 2016). fNIRS is a very attractive method for these applications, because it is a non-invasive, portable and low cost technique, compared to methods like functional magnetic resonance imaging (fMRI), while also relying on the blood-oxygen-level dependent (BOLD) effect(Ogawa, Lee, Kay, \& Tank, 1990). The increased usage of fNIRS for $\mathrm{HCl}$ and $\mathrm{BCl}$ applications is only possible because previous research improved the signal quality and reliability of fNIRS measurements(Boas et al., 2003). A lot of the $\mathrm{BCl}$ research in this field was based on individualized analyses using various machine learning methods such as support vector machines (SVMs) or other classifiers(Naseer \& Hong, 2015). While these methods allow the use of $\mathrm{BCls}$ they are usually not providing insights in the signal quality and trial by trial performance of the participant. We developed a software package called Turbo-Satori bridging the gap between comprehensive analysis methods and the detailed inspection of fNIRS signals in realtime during an ongoing experiment. This possibility is very important since it allows real-time quality assurance by inspecting individual fNIRS channels and the overall signal quality of all channels at the same time. Especially when using neurofeedback routines, the quality of individual channels is crucial because they can be used as the basis of the neurofeedback signal, translated for example to the level of a thermometer display(Krause et al., 2017; Weiskopf et al., 2003). But also in other $\mathrm{BCl}$ applications, transparent information and statistical data about the signal quality of individual channels is important to relate it to the overall performance. Therefore, the mentioned features allow several potential use cases for Turbo-Satori requiring minimal implementation time because of the available interfaces and clearly structured user interface. We provide different use case examples for Turbo-Satori including SVM based left right finger tapping 
discrimination, neurofeedback example experiments as well as $\mathrm{BCl}$ application examples. Another aspect is the usability of the software which needs to fulfill the requirement of integrating and controlling complex routines while assuring its ease of use. Such a system allows even early stage researches to perform advanced real-time fNIRS based $\mathrm{BCl}$ experiments with relatively little effort. In this article, we will cover all major aspects of the software starting with the unique user interface and controls followed by specific preprocessing and analysis routines. 


\section{METHODS}

Turbo-Satori is written in $\mathrm{C}++$ and uses different libraries and frameworks such as Qt(Qt, n.d.) and Eigen(Guennebaud, Jacob, \& others, n.d.) as well as a collection of useful $\mathrm{C}++$ classes for digital signal processing("A Collection of Useful $\mathrm{C}++$ Classes for Digital Signal Processing," n.d.) .While developed for cross-platform use, the software currently runs only on the Windows platform in combination with devices from the company NIRx (NIRx Medical Technologies, LLC, 5670 Wilshire Blvd Suite, 1800, Los Angeles, CA 90036 U.S.A). The next section describes the user interface of Turbo-Satori that is designed to focus mainly on rich time course information and ease of use, especially for research investigating individual fNIRS channels.

\section{User Interface}

The user interface of Turbo-Satori mainly consists of four parts with specific settings and options. These interface parts can be freely rearranged or be hidden to be able to limit the data representation to only the currently desired information. A screenshot of the running application is shown in Fig. 1. The first part of the interface (see number 1 in Fig. 1) allows the user to configure global settings of analysis, controls and plotting. It also contains the control settings to start / stop the real-time, online or offline analysis. On the left side of the interface all available channels are displaying the beta value of each channel at the current point in time using the currently defined contrasts. The arrangement of the channels starts with first source and first detector in the top left corner to source and detector $\mathrm{n}$ in the bottom right like the definition in the NIRStar data acquisition software allowing to immediately observe whether the expected pattern is present ensuring data quality. 


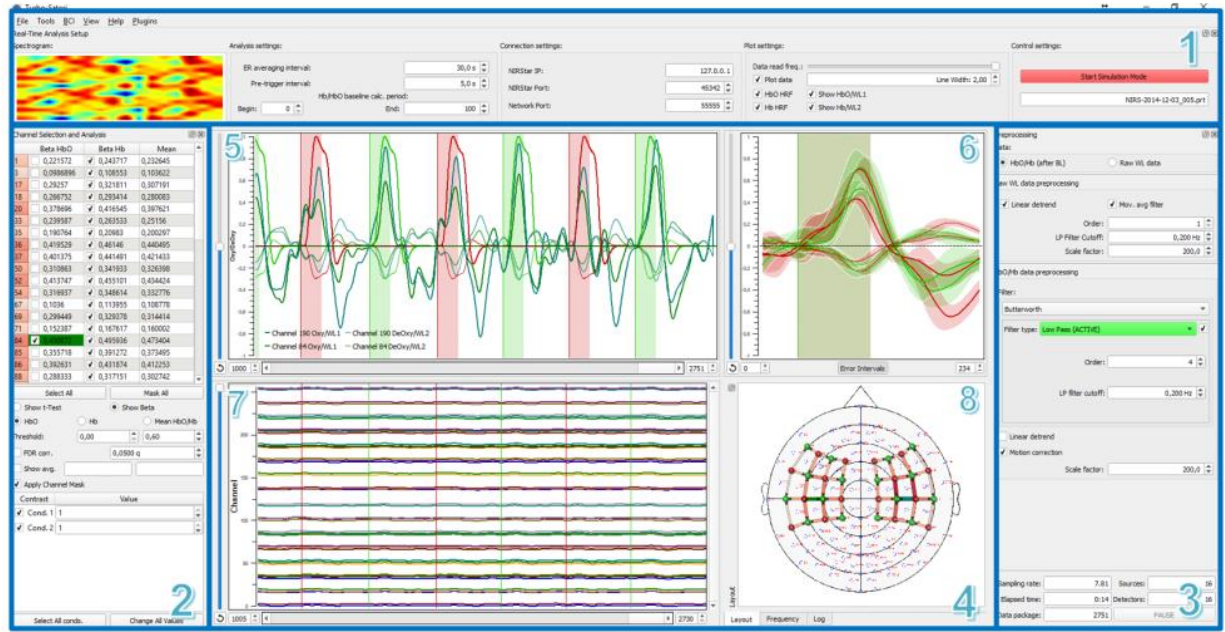

Figure 1: The graphical user interface of Turbo-Satori. The interface consists of four parts: 1 . The real-time analysis setup window, which includes configurations to set up the real-time analysis as well as connection, plotting and control settings. 2. The channel selection and analysis window. It contains the basic parameters and table to perform real-time analysis of the available channels. 3. The preprocessing window allows to change the preprocessing parameters for raw wavelength data as well as for the $\mathrm{HbO} / \mathrm{Hb}$ converted data. Additionally, the status information is shown in the bottom of the window. 4. The main application window provides three different plots and a layout view, frequency spectrogram and log window area (numbers 5-8) showing useful information or the layout of the sources, detectors and channels. 5. The time course of the selected channels (or average) for Raw wavelength 1 and/or 2, $\mathrm{HbO}$ and/or $\mathrm{Hb}$ signal. 6. The event related average of the selected channels time course. 7. The combined plot for all marked channels. 8 The layout view, (layout shown in figure, see tab bar at bottom).

The second part of the interface covers the channel selection for the plotting interface using a standard table which also shows basic statistical analysis results allowing to inspect beta-value of the oxyhemoglobin $(\mathrm{HbO})$ and deoxy-hemoglobin $(\mathrm{Hb})$ time courses in individual channels based on a real-time general linear model (GLM) (see below) calculation. It is also possible to average selected channels and get a separate analysis and plot for the average. In the bottom of 
this interface section one can set specific contrasts of each available condition defined in the experiment. Part three of the interface handles the preprocessing of the raw or converted $\mathrm{HbO} / \mathrm{Hb}$ data. More details about the filtering options are described in the next sub-section of the article. In the bottom of this interface section basic information about the experiment and settings are available.

Most of the interface (see number 4 in Fig. 1) is used to visualize and inspect time course data. There are two different types of data shown. The first one is presented in the top left (individual time course of selected channels, number 5 in Fig. 1), top right (event related averages of each condition for each selected channel, number 6 in Fig. 1) and bottom left (combined overview of all channels, number 7 in Fig. 1) of this interface section. These parts represent the individual time course of the channels and allow to inspect each channel individually while at the same time provide an overview of all channels which makes it possible to check for overall artifacts in the data or in individual channels. The event related average plot gives more insights in condition-based response profiles allowing to compare different tasks as well as overall performance and data quality.

In the bottom right of this section a layout overview is presented which shows the sources, detectors and the respective channels on a predefined montage (number 8 in Fig. 1). The source and detector positions are stored with respect to the underlying montage image and can be individually adapted using an integrated layout manager or loaded from the stored information if re-analyzing a dataset (stored with NIRStar software bundled using a NIRx device).

The configuration of the sections 1 to 3 is very flexible allowing to choose where to place each part or to have it as a floating interface for multi-screen configurations.

\section{Real-time preprocessing}

In comparison to offline analysis the major constraint of real-time analysis is to achieve a constant calculation time from time point to time point that is shorter than the sampling interval of two consecutive measurements during the whole experiment. This requirement is especially important for neurofeedback and $\mathrm{BCl}$ experiments using data from extended time windows or even the full-time course to calculate values for visualization and classification. The constant calculation time 
constraint is required for all sub-parts in the moment-to-moment processing pipeline including I/O operations, conversion from raw to $\mathrm{HbO} / \mathrm{Hb}$ signals, preprocessing, analysis and neurofeedback / $\mathrm{BCl}$ calculation and presentation, and the sum of operation times of these steps must be smaller than the sampling interval.

Turbo-Satori provides online $\mathrm{HbO} / \mathrm{Hb}$ concentration value calculations from raw wavelength data using the modified beer lambert law (MBLL)(Delpy et al., 1988). The parameters for concentration changes are based on the work of Essenpreis et. al. who performed a study on adults to calculate the across-subject average differential path length (DPF) (Essenpreis et al., 1993) and the molar extinction coefficients $(\varepsilon$ ) from W. B. Gratzer (W. B. Gratzer, n.d.), see Table 1.

Table 1: Parameters to calculate concentration changes using MBLL.

\begin{tabular}{l|ccc}
\multicolumn{2}{c}{$D P F^{\lambda}$} & $\varepsilon_{H b O_{\lambda}}\left(\frac{l}{\mathrm{~cm} \mathrm{mmol}}\right)$ & $\varepsilon_{H b_{\lambda}}\left(\frac{l}{\mathrm{~cm} \mathrm{mmol}}\right)$ \\
\hline $\begin{array}{l}\lambda_{1} \\
=760 \mathrm{~nm}\end{array}$ & 6.40 & 1.4865865 & 3.843707 \\
& & & \\
$\lambda_{2}$ & 5.75 & 2.526391 & 1.798643 \\
$=850 \mathrm{~nm}$ & & &
\end{tabular}

Since the $\mathrm{HbO} / \mathrm{Hb}$ concentration values need a baseline of raw data before it can be calculated in real-time for future time points, the program initially shows raw data and switches to $\mathrm{HbO} / \mathrm{Hb}$ data display as soon as the baseline is available. As default, the baseline is calculated from the first 200 received values but this can be changed using the "Hb/HbO baseline calc. period, Begin:" and "End" fields in the "Analysis Settings" setup dialog. Raw wavelength and converted $\mathrm{HbO} / \mathrm{Hb}$ data has its own preprocessing sub-panel. The values of the full channel plotter and especially of the selected (and event-related) channel plotter should be scaled so that they fall within -1 and +1 (the range of the $y$ axis). Preprocessing options allow to detrend the raw data and apply a moving average based low-pass filter with an order up to five. The order defines a repeated application of the low-pass filter on the data for stronger 
high frequency noise removal. The $\mathrm{HbO} / \mathrm{Hb}$ data can be low- and highpass filtered in real-time using a variety of different infinite impulse response (IIR) filters like the exponential moving average (EMA) filter and a simple moving average (SMA) filter. Implementations of RBJ Biquad, Butterworth, Chebyshev, Elliptic, Bessel and Legendre filter designs are based on the work of ("A Collection of Useful C++ Classes for Digital Signal Processing," n.d.) including Low Pass, High Pass, Band Pass and Band Stop transformations for most of the filters. This allows to adapt the filtering to each participant separately giving the individual advantages and drawbacks of each filter type(Antoniou, 2006; Popescu, 1996).

To verify the effect of the used filtering settings a frequency spectrogram is available showing the magnitude response for frequencies in a range from $0.0 \mathrm{hz}$ to $1.4 \mathrm{hz}$. The frequency plot is updated in real-time and displays the frequency spectrogram of the selected channel for $\mathrm{HbO}$ and/or $\mathrm{Hb}$. In addition to the real-time filtering procedures a motion correction approach described in (Cui, Bray, \& Reiss, 2010) is implemented which is based on the negative correlation of oxygenated and deoxygenated hemoglobin dynamics(Cui et al., 2010). This approach is very well suited for real-time calculation because of three major characteristics which are essential for real-time signal quality improvement: The correction at a specific point in time does only rely on the data before and does not need data later than the current point, the computation time for this procedure is low and constant and it does not require user interaction because the method can be used in an automatic fashion(Cui et al., 2010). The method is applied after the MBLL and filtering of the data. The motion correction can be turned on using the motion correction check box in the $\mathrm{HbO} / \mathrm{Hb}$ preprocessing field. For further performance details please recite (Cui et al., 2010).

\section{Real-time data analysis}

The analysis performed in Turbo-Satori is based on a real-time implementation of the GLM, the recursive least squares GLM (rlsGLM)(Pollock, 1999). Fitting an ordinary least squares (OLS) GLM is the same as finding estimates of the beta values minimizing the sum of squared error values $(e)$. $y$ represents the $\mathrm{HbO}$ or $\mathrm{Hb}$ data. 


$$
\begin{aligned}
& e^{\prime} e=\sum_{t=1}^{N} e_{t}^{2}=(y-X \beta)^{\prime}(y-X \beta), \\
& =y^{\prime} \mathrm{y}-2 \beta^{\prime} X^{\prime} \mathrm{y}+\beta^{\prime} X^{\prime} X \beta, \\
& \frac{d s}{d \beta}=-2 X^{\prime} \mathrm{y}+2 X^{\prime} X \beta=0,
\end{aligned}
$$

The solution can be directly calculated as:

$$
\beta=\left(X^{\prime} X\right)^{-1} X^{\prime} y
$$

For the rlsGLM, the beta values $(\beta)$ and inverted $X^{\prime} X$ design matrix can be updated incrementally using only information of the new time point with the following recursive equations:

$$
\begin{gathered}
\beta_{t+1}=\beta_{t}+\left(X_{t}^{\prime} X_{t}\right)^{-1} \frac{\left(y_{t+1}-x_{t+1} \beta_{t}\right)}{1+x_{t+1}^{\prime}\left(X_{t}^{\prime} X_{t}\right)^{-1} x_{t+1}}, \\
\left(X_{t+1}^{\prime} X_{t+1}\right)^{-1}=\left(X_{t}^{\prime} X_{t}\right)^{-1}-\frac{\left(X_{t}^{\prime} X_{t}\right)^{-1} x_{t+1} x_{t+1}^{\prime}\left(X_{t}^{\prime} X_{t}\right)^{-1}}{1+x_{t+1}^{\prime}\left(X_{t}^{\prime} X_{t}\right)^{-1} x_{t+1}},
\end{gathered}
$$

Since the $\left(X^{\prime} X\right)^{-1}$ term is the same for all channels, it can be precomputed before solving the $\beta$ for individual channels reducing the calculation time for each new data point in time.

In its standard formulation, rlsGLM result in the same beta estimates as a standard GLM over the whole time course up to the current point in time.

The GLM is calculated for each channel individually and for the average of the selected channels. The resulting beta values of the calculations are represented in the channel selection and analysis window as values as well as color coded in each channels row header. The user can change the thresholds for the color coding using the threshold spin boxes below the table. This allows a quick inspection of all channels and makes it easy for the user to select the most promising channel. A source of confounding noise in fNIRS analysis are serial correlations. Recent studies have proposed different methods to correct for this noise using offline(Cui et al., 2010; Naseer \& Hong, 2015) and real-time(Barker, Rosso, Sparto, \& Huppert, 2016) data analysis. In this real-time analysis approach the computed beta values are used for qualitative judgments, 
which are unaffected by serial correlations. The GLM may also be used to estimate single-trial responses (beta values) in the context of classifier applications (see below).

The results are also shown in the connection color and strength of a source and a detector in the layout view. This view allows a spatial inspection of the activation on a predefined montage or image. In some cases, it is even useful to take a picture of the fNIRS cap on a participant's head and point the sources, detectors and channels onto this image directly allowing an immediate translation from activity patterns to source detector locations on the participants head.

\section{Real-time multi-variate pattern classification}

Multi-variate pattern classification (MVPC) is gaining increasing interest in the neuroimaging community for both, offline and real-time fNIRS data analysis, because it allows detecting differences between conditions with higher sensitivity than conventional univariate analysis (see above) by focusing on the analysis and comparison of distributed patterns of activity. In such a multi-variate approach, data from many sources (e.g., channels in fNIRS and EEG, voxels in fMRI) are jointly analyzed. The high sensitivity of MVPC allows "brain reading" applications that aim to decode (predict) specific mental states or representational content from activity patterns. After performing a training phase, the decoding/prediction phase requires little computational load and it is, thus, suitable for real-time $\mathrm{BCl}$ applications including the decoding of mental states. Turbo-Satori uses multi-variate pattern classification based on the widely used support vector machine (SVM) learning algorithm. The used library in Turbo-Satori is the LIBSVM library(Chang \& Lin, 2013). In the "Tools" menu, the "SVM Training" item can be used to open the "Multi-Channel Pattern Classification" dialog allowing to train a SVM on the data from one or more completed runs of a real-time session (currently only one training run is supported); the dialog can also be used to perform offline testing, e.g., on the data of a subsequent run. After the training phase, the "Real-Time SVM Classification" dialog can be used to start trial-by-trial online classification producing prediction values that indicate to which class a distributed activity pattern belongs according to the information extracted by the classifier. 
The "Multi-Channel Pattern Classification" dialog as shown in Fig. 2 can be used to train SVMs to associate distributed activity patterns with class labels that correspond to two or more conditions ("classes") of a paradigm.

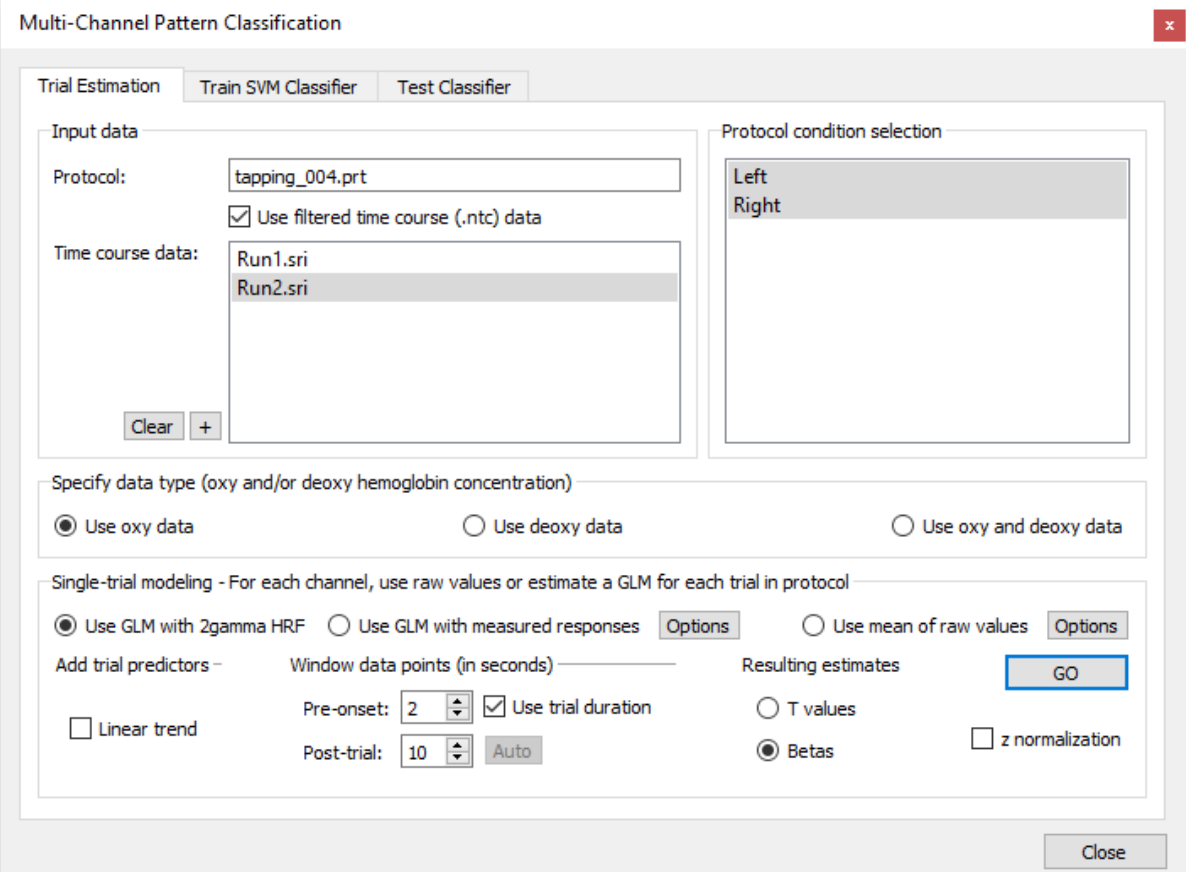

Figure 2: Multi-Channel Pattern Classification dialog. The Trial Estimation tab is selected allowing to control the estimation of response values per trial.

The "Trial Estimation" tab (Fig. 2) is used to specify how responses should be estimated for individual trials at each channel. The "Train SVM Classifier" tab (see Fig. 3) is used to create training data suitable for SVMs and to perform the actual training process. 


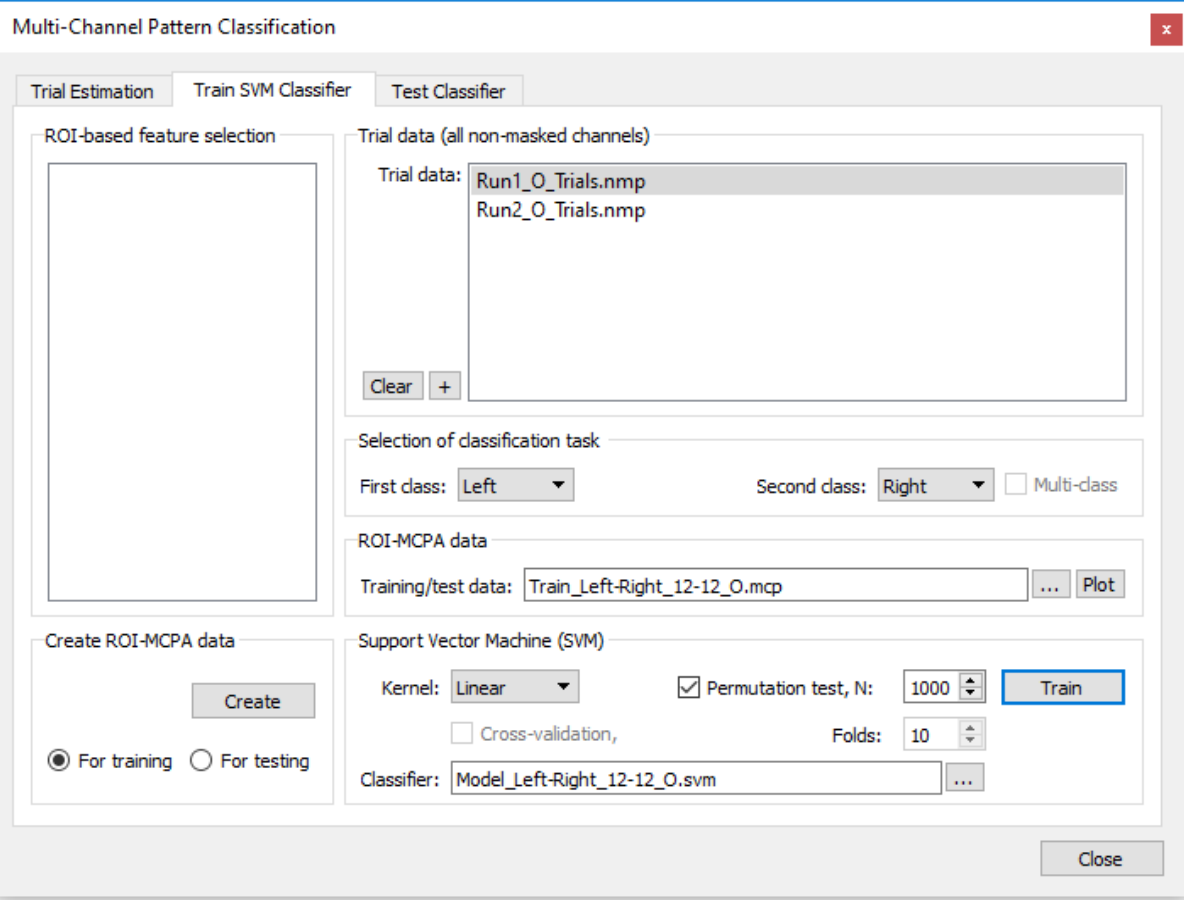

Figure 3: Multi-Channel Pattern Classification dialog. Here the Train SVM Classifier options are shown enabling to specify the classification task and channels used for training.

The classifier supports multiclass problems, i.e., distributed patterns may be assigned to two or more different conditions. The trained classifier can then be tested on completed run data using the "Test Classifier" tab. For real-time classification, the "Real-Time SVM Classification" dialog is used. In order to obtain input patterns for different classes, response values are estimated for each individual trial using a standard OLS-GLM (see above). Estimated single-trial responses across relevant channels (e.g., all non-masked channels of a montage) then form the feature vectors used to train or test the classifier. An estimated trial response might be as simple as the activity level at a certain time point (e.g., at the time of the expected hemodynamic peak response) or the mean response of a few measurement points around the peak response relative to a pre-stimulus baseline. In Turbo-Satori, however, trial-related time points are integrated by running single-trial GLMs (see above) to estimate beta values using an expected hemodynamic response shape. The estimated single-trial responses 
(beta values) across channels form multi-variate patterns that can be used for training runs and for online classification at the end of a trial. For a more robust fit of the model, a linear trend confound predictor may be added (next to the constant predictor and the main predictor) for estimating (and removing) a linear trend. The single trial channel estimates may be specified as either $t$ values, beta values or percent signal change beta values. The beta values option is selected as default. As default, the time course data within each trial window is znormalized, which can be turned off if raw time courses should be used. The "Plot" button can be used to create a visualization of the created training data (see results section for snapshot).

To test the performance of the classifier we conducted an experiment using a finger tapping paradigm described in section 2.6, including two training runs and one test run. A subset of 8 sources and 8 detectors incorporating 20 channels from the finger tapping paradigm was used in this experiment. The subset includes source $2,3,4,6,12,13,14,16$ and detectors 1,2,4,5,11,14,15,16, of the used sources and detectors described in section 3.1. The channel number is based on subsets source-detector pairs sorted in increasing numerical order (e.g. Channel \#1: Source 2, Detector 1, Channel \#2: Source 2, Detector 2,...). The first 10 channels (1-10) are placed on the left hemisphere and the 10 least channels (11-20) are placed on the right hemisphere. Only these 20 channels were used to train the classifier. A portable fNIRs system (NIRSport 8x8, NIRx Medizintechnik GmbH, Berlin, Germany) was used in combination with a fNIRs cap having proper size for each individual participant. The sampling rate was $7.8125 \mathrm{~Hz}$.

\section{Neurofeedback calculation}

For many (clinical) neurofeedback applications, it is desirable to visualize, e.g. in a thermometer, the mean activation level from selected channels during a modulation period relative to a baseline level, and to present the activity visualization to the participant as feedback. The Neurofeedback dialog, Fig. 4, can be used to select one channel or the average of multiple channels for feedback calculation and visualization and to prepare details about the feedback display. 


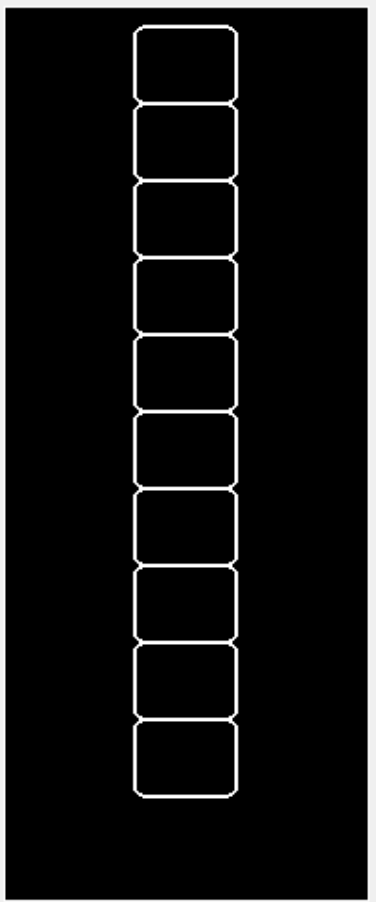

Feedback type

- Thermometer

Time course plot

Delayed feedback

No activity output to subject

V Export numerical values to disk

Export image snapshots to disk

Feedback settings

Average feedback values:

Max Val Oxy: $0,50 \neq$ Deoxy 0,20 ㄴ

$\square$ Set to max measured

Use ROI-GLM baseline (b0)

Baseline display level: 0

$\checkmark$ Show condition name as instruction

Duration: $1,5 \mathrm{~s} \rightarrow$
Based on time course / classifier

- Averaged selected channels

SVM output

- Use oxy data

Use deoxy data

Use oxy + deoxy data

Multi-ROI integration

Average multiple ROIs

Visualize multiple ROIs

Feedback baseline data points

Condition: MentalDrawi $\mathbf{v}$

Shift at begin: $5 \stackrel{\text { s }}{5}$

Shift at end: $2 \geqslant \mathrm{~s}$

No feedback condition

Figure 4: The neurofeedback dialog.

In this section, details about the calculation of neurofeedback signals from selected channels are described and how calculation is controlled using options in the "Feedback settings" field in the Neurofeedback dialog. Values for neurofeedback are calculated by subtracting the last baseline (BL) from the current scaled oxy/deoxy value. Note that deoxy values are inverted so that a negative deflection results in a positive feedback signal. In detail, the calculations are as follows:

$$
\begin{gathered}
f b_{\text {oxy }}=\left(o x y_{\text {value }}-o x y_{B L}\right) \\
f b_{\text {deoxy }}=-1 *\left(\text { deoxy }_{\text {value }}-\operatorname{deoxy}_{B L}\right)(12) \\
f b_{\text {combined }}=\frac{1}{2} *\left(\left(o x y_{\text {value }}-o x y_{B L}\right)-\left(\text { deoxy }_{\text {value }}-\text { deoxy }_{B L}\right)\right)
\end{gathered}
$$


Please note that equation (11) to (13) assume a negative correlation between the patterns of $\mathrm{HbO}$ and $\mathrm{Hb}$. This results in a percent signal value that is used to fill the thermometer display. To convert this value into a corresponding fill level of the thermometer, the resulting value is related to the "Max Val Oxy" and "Deoxy" values as specified in the Max Val Oxy and Max Val Deoxy field. If, for example, the calculated $f b_{\text {oxy }}$ value is 0.25 and the Max Val Oxy value is set to 0.5 , the thermometer is filled half, i.e. 5 out of 10 rectangles will be filled since $\mathrm{fb} /$ max_val $_{\text {oxy }}=0.5$. To find good values for the maximum, it might be useful to observe achievable neurofeedback amplitudes for specific channels in localizer or test runs.

Because of the sluggishness of the fNIRS (BOLD) response, the values defined by a baseline interval should not be used as defined in the protocol directly but should take the hemodynamic delay into account. In order to protect the BOLD decay from a previous modulation condition, values at the begin of the baseline condition need to be excluded. The number of points to exclude can be specified with the "Shift at begin" spin box. If the previous baseline condition occurred, for example, from time points (in seconds) 50 - 70 (20 seconds), the actual values considered for baseline calculation would be from 55 - 70 in case of value 5 for the shift at the begin of the baseline interval. In a similar way, one can also extend points "to the right side" at the end of a baseline interval since it takes some time until the BOLD signal rises in the modulation block. Since it is expected that the signal starts to rise already after about 2-3 seconds, the shift into the modulation interval should be, however, smaller than at the begin of the baseline condition. Using one second for the shift at end parameter results in the final interval 55-71 for the baseline period. The signal values of the identified data points within this period (16 seconds in the example) will be averaged to obtain the baseline level bl for the subsequent modulation block (see above). Note that 4 seconds are minimally required for a premodulation baseline (at least 10 are recommended), otherwise no feedback output is produced in the thermometer display.

\section{Real-time preprocessing and analysis verification}

We used data from four experiments to verify the calculation times for the whole preprocessing and analysis using second order real-time Butterworth low- and high-pass filters and rIsGLM calculation with two 
main predictors. The four experiments were analyzed post hoc using the same routines as used during real-time measurements allowing a direct translation. The first experiment (Fig. 8, yellow, 20 Channels [10.4167Hz]) consisted of 224990 data points which were generated automatically simulating an 6-hour experiment. The second experiment (Fig. 8, blue, 64 channels $[7.8125 \mathrm{~Hz}]$ ) was a mental drawing paradigm which consisted of 3291 data points and took approximately 7 minutes. The third and fourth experiment were data from the NIRx support website("NIRx support website," n.d.) including both a left or right hand finger tapping experiment. The first of these two experiments (Fig. 8, orange, 256 channels $[7.8125 \mathrm{~Hz}]$ ) consisted of 2750 data points and took approx. 6 minutes and the second experiment (Fig. 8, gray, 256 channels $[3.91 \mathrm{~Hz}]$ ) consisted of 2460 data points which were approx. 10 minutes. These two datasets were also used to show the performance of the rlsGLM and user interface as describe in the results section.

\section{Experimental setup}

$B C l$ and especially Neurofeedback experiments can require a complex setup involving multiple devices and software. It is therefore important to clarify the connections from and to Turbo-Satori as well as the position in an example setup as shown in Fig. 5. 


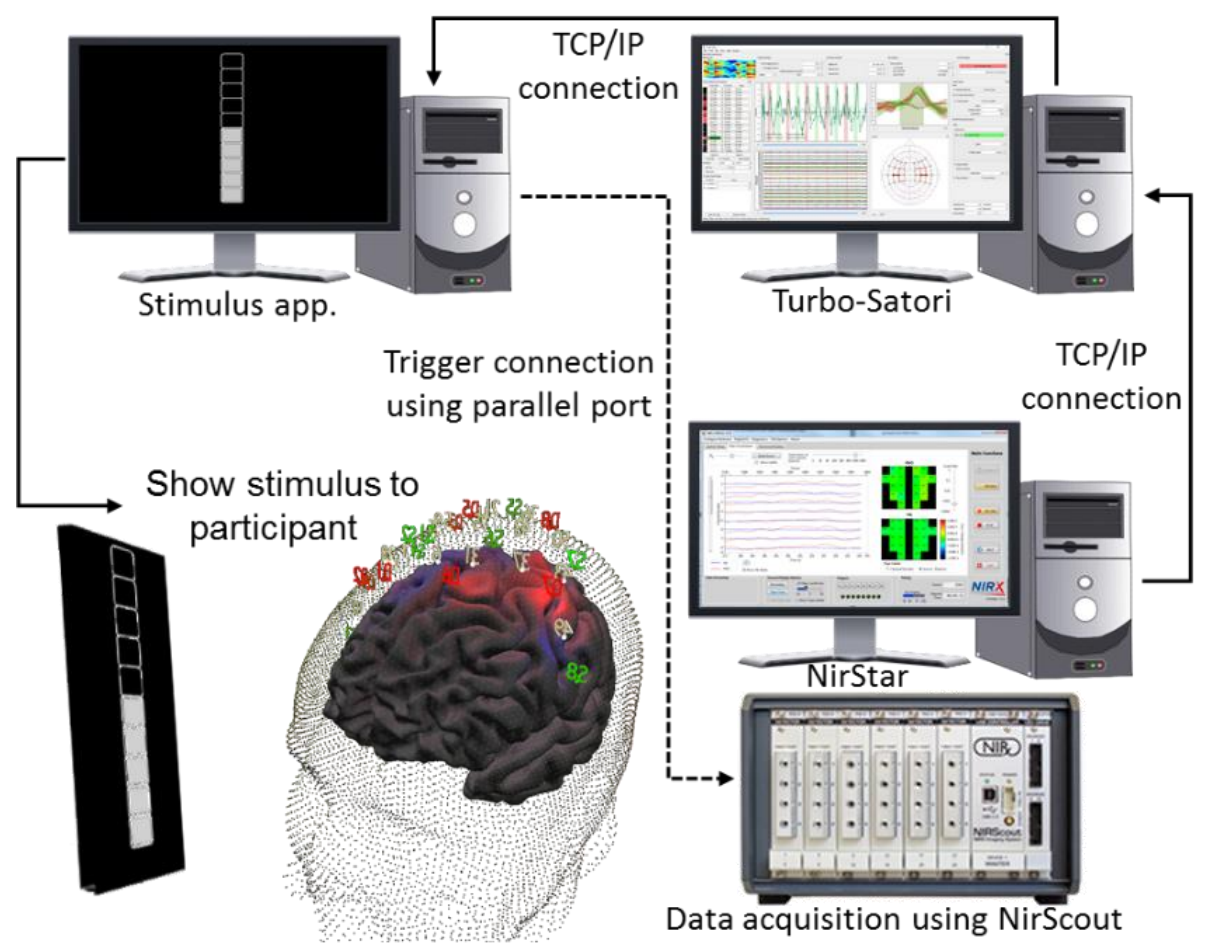

Figure 5: Setup of real-time fNIRS neurofeedback experiment using Turbo-Satori.

The data acquisition is based on a NIRx fNIRS system (NIRScout, NIRSCOUTX or NIRSport) which is operated by a computer or laptop running NIRStar (version 14.2)("NIRx support website," n.d.). The NIRx device is connected via a Universal Serial Bus (USB) cable to the computer with NIRStar. Turbo-Satori is installed on a separate computer which connects to the computer running NIRStar via a TCP/IP Ethernet connection. The two software packages use the NIRx network SDK to communicate in real-time and exchange data between NIRStar and Turbo-Satori. For $\mathrm{BCl}$ or specifically neurofeedback applications the stimulus presentation software needs to read or receive the $\mathrm{BCl}$ or neurofeedback information in real-time. In this example setup, the stimulus presentation software also runs on a separate computer. This computer is also connected to the computer running Turbo-Satori using a TCP/IP based Ethernet network connection. The data is exchange using a self-designed network interface enabling real-time access to raw and preprocessed fNIRS data as well as protocol and statistical 
information. This interface is available for software tools like Expyriment (Krause \& Lindemann, 2014), BrainStim ("BrainStim," n.d.) or MATLAB (MATLAB, The MathWorks Inc., Natick, MA, 2000). The prepared stimuli and $\mathrm{BCl}$ or neurofeedback information are then presented to the participant.

The separation of all three software tools ensures a fast processing of each element in the chain and ensures compliance to the time critical requirements of these kind of experiments. 


\section{RESULTS}

\section{Finger tapping example}

The rlsGLM analysis revealed different patterns for left and right finger tapping visualized in the layout view of Turbo-Satori. The task specific patterns on the right hemisphere for left finger (red task period) tapping and on the left hemisphere for right finger tapping (green task period) appears after three task epochs on the right hemisphere during the left tapping task and after four task epochs on the left hemisphere for the right tapping task using a fixed beta threshold. The minimum beta threshold was set to 0.40 (white color and thin lines) and the maximum color coding (red) and strength was set to 0.70. For both tasks, a separate task vs rest contrast was selected. After each task block a state of the current layout was extracted and put together in Fig. 6 .

After a few repetitions of each task one can see a pattern evolving representing the channels exceeding the selected beta threshold. Also, a difference in the pattern strength is visible; in Fig. 6 a) the right hemisphere for left finger tapping and in Fig. 6 b) the left hemisphere for right finger tapping exhibits a larger response strength. The beta values of the offline analysis were highly correlated compared to the online analysis having an average Pearson correlation coefficient of 0.999 being significant with $\mathrm{P}<0.0001$. Comparing the neurofeedback levels and the underlying preprocessed fNIRS signal (for $\mathrm{HbO}, \mathrm{Hb}$ and mean) resulted in an average Pearson correlation of 0.9311 being significant with $\mathrm{P}<0.0001$. 


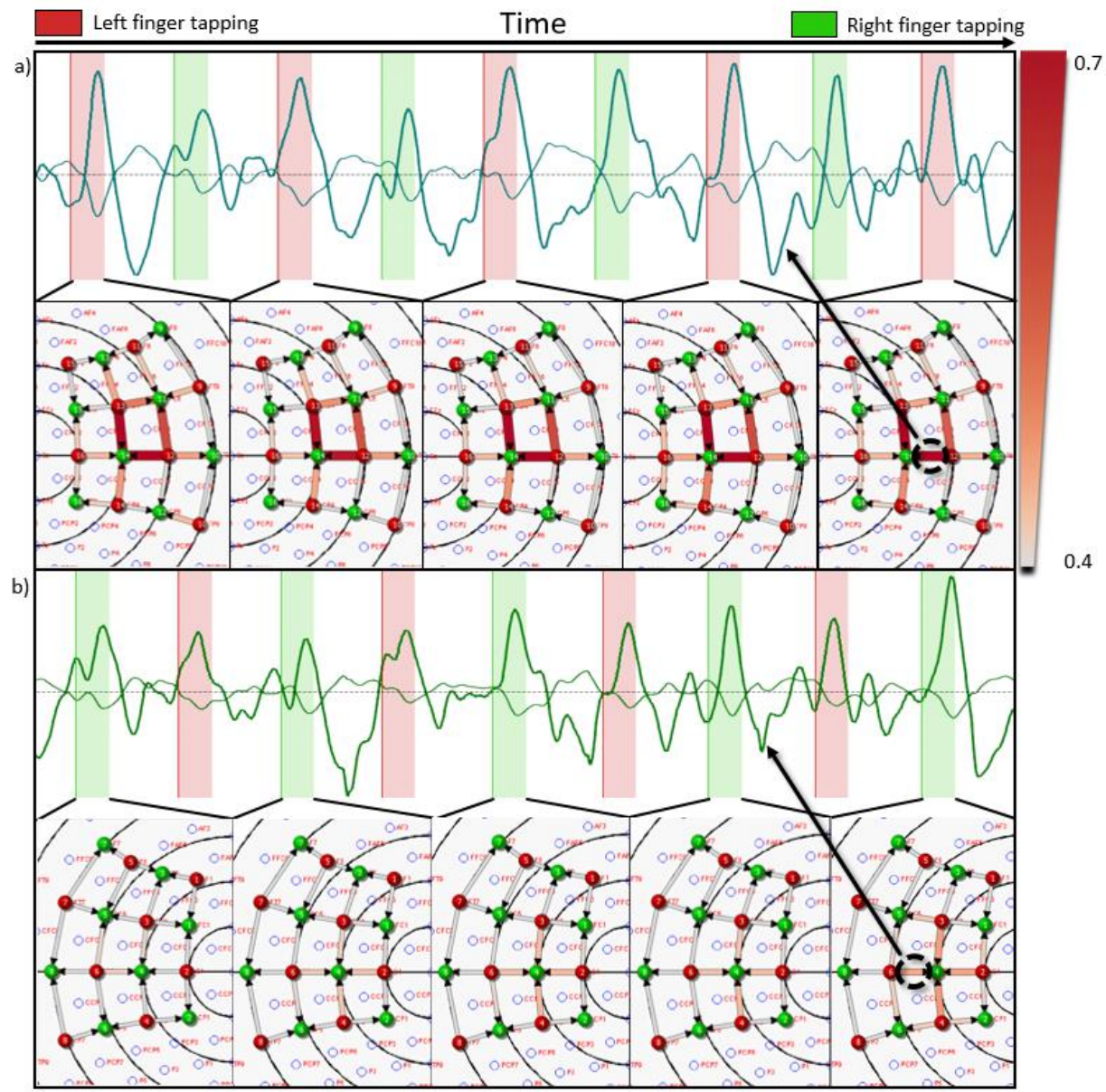

Figure 6: Incremental results of one of the left and right finger tapping experiments. a) represents the right hemisphere pattern and show the oxy (bold line) / deoxy (thinner line) time course of the channel covering C4. b) shows the left hemisphere pattern and oxy (bold line) / deoxy (thinner line) time course of the channel covering $\mathrm{C} 3$. The strength of the edges between sources (red points) and detectors (green points) represent the beta values using the $\mathrm{HbO}$ time courses for the GLM calculation in this example.

\section{SVM analysis of finger tapping experiment}

In Fig. 7 the trained classifier is shown. The y-axis represents the training patterns with black lines separating the different classes for each run (first run class 1 , first run class 2 , second run class 1 second run class 2 ); 
the $x$-axis represents the channels used for training (20 channels in this example). The colored rectangles indicate the value of the estimated responses within a pattern for a given channel (negative values greento-blue, positive values yellow-to-red). The rows at the bottom of the graph show the mean values averaged over the trials of the available classes. In this example, one can clearly see in the average plot a pattern in the trial responses. For the first class (left finger tapping) the least 10 channels (11-20, placed on top of the right hemisphere) show a stronger response and the first 10 channels (1-10, placed on top of the left hemisphere) show a larger response for the second class (right finger tapping).

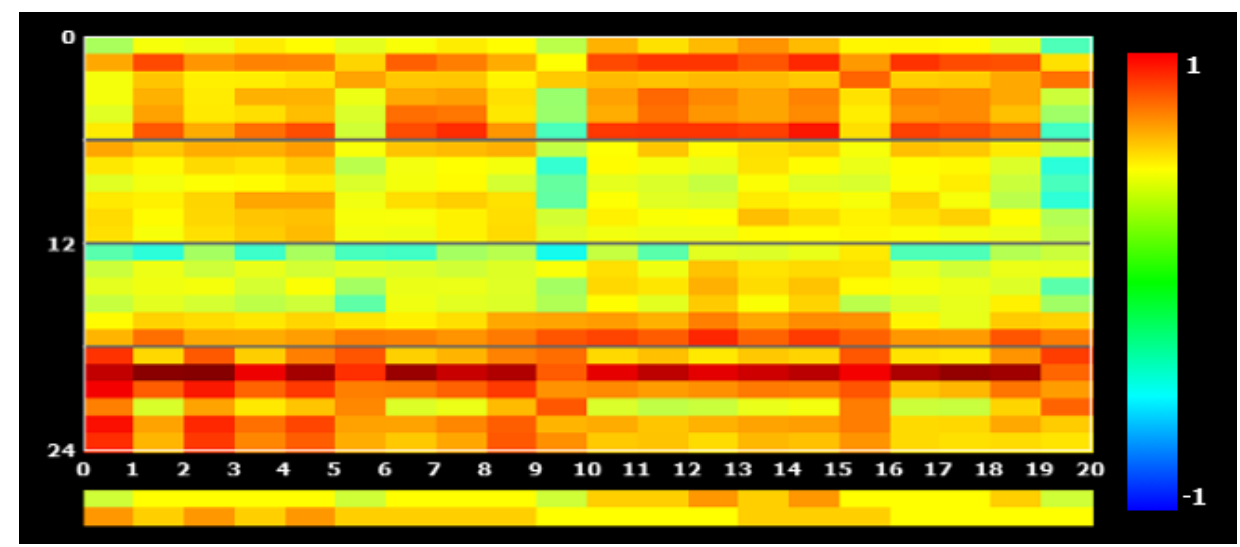

Figure 7: Visualization of the finger tapping training dataset in the training pattern plot. Rows in the upper part represent patterns of single trials with black lines separating different classes and runs (first run class 1 , first run class2, second run class 1 second run class2). The colored rectangles indicate the value of the estimated responses within a pattern for a given channel (negative values green-to-blue, positive values yellow-to-red). The rows at the bottom of the graph show the mean values averaged over the trials of the two available classes (left and right tapping).

We employed a leave-one-run-out splitting procedure to cross-validate the classification performance between left and right finger tapping using a 1000 folds' permutation test. For this dataset using two training runs ( 12 trials per condition) and one test run an accuracy of $100 \%$ was reached having a significant accuracy $(p<0.01)$. The 95th percentile of each 1000 permutations test was $69.36 \%$. 


\section{Calculation times}

All experiments were analyzed successfully in Turbo-Satori using a standard laptop computer with $3.0 \mathrm{GHz}$ Intel ${ }^{\circledR}$ Core $^{\mathrm{TM}}$ i7-5500U CPU, 8GB ram and $512 \mathrm{mb}$ SSD storage. The calculation times were much lower than the respective sampling rates. For the first experiment an average calculation time of $0.361 \mathrm{~ms}$ ( $\mathrm{min} 0.246 \mathrm{~ms}$, $\max 2.249 \mathrm{~ms}$, std. 0.0841) was achieved using a sampling rate of $10.41467 \mathrm{~Hz}$ ( $\sim 96 \mathrm{~ms}$ interval). The second experiment was performed with a mean calculation time of $0.741 \mathrm{~ms}$ ( $\mathrm{min} 0.443 \mathrm{~ms}$, $\max 4.480 \mathrm{~ms}$, std. 0.164) with a sampling frequency of $7.8125 \mathrm{~Hz}$ (128ms interval). For the third experiment, a calculation time of $2.661 \mathrm{~ms}$ ( $\mathrm{min} 1.496 \mathrm{~ms}$, $\max 15.544 \mathrm{~ms}$, std. 0.509) was achieved using a sampling rate of $7.8125 \mathrm{~Hz}$ (128ms interval). The last experiment performed similarly having an average calculation time of $2.753 \mathrm{~ms}$ ( $\mathrm{min} 1.495 \mathrm{~ms}$, max $16.712 \mathrm{~ms}$, std. 0.503 ) using a sampling rate of $3.91 \mathrm{~Hz}(\sim 255 \mathrm{~ms}$ interval). All experiments didn't show any significant linear increase in calculation time over the whole dataset (see asterisks in Fig. 8). An overview of these results is shown in Fig. 8.

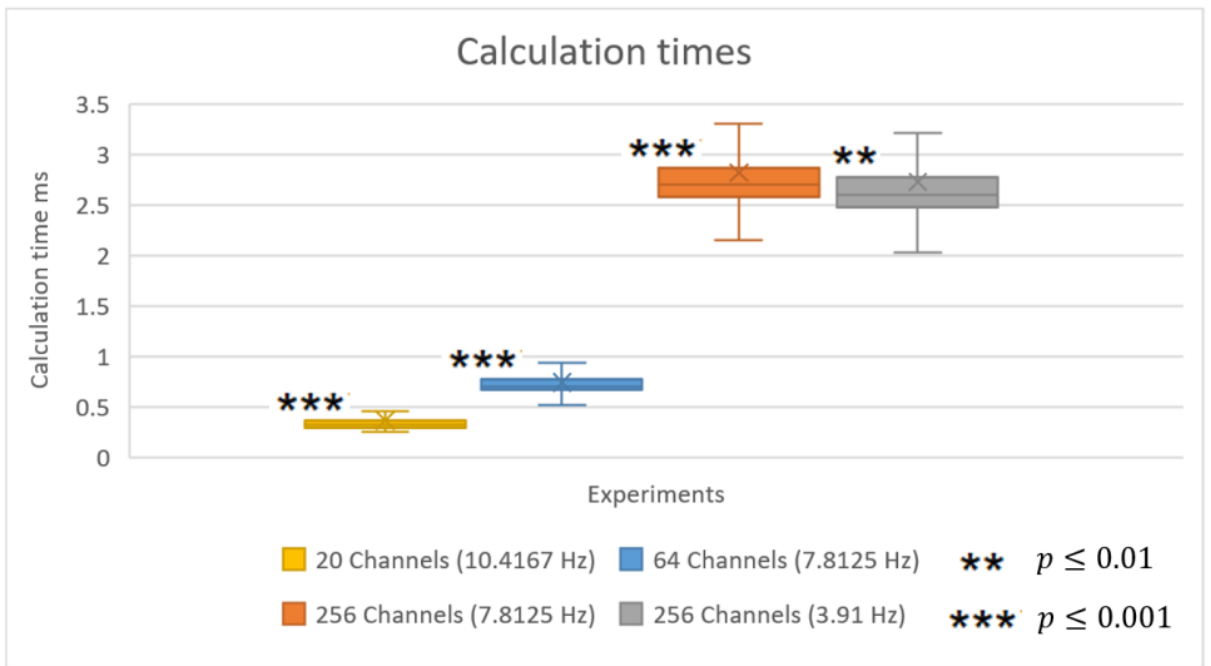

Figure 8: Calculation times for the 4 conducted experiments with varying number of channels and sampling rate. Asterisk indicate no trend in calculation times. 


\section{DISCUSSION}

$\mathrm{BCls}$ and especially neurofeedback applications using fNIRS gain increasing importance in recent years. Therefore, software is needed that helps researchers and clinicians to easily use state-of-the-art analysis methods and to apply these techniques in broad research and increasingly in clinical fields. Turbo-Satori has been developed to fulfil these needs by integrating a fast calculation engine implemented in $\mathrm{C}++$ with an easy to use interface that provides useful information about channel-level signal quality. The software offers analysis methods including statistical and classification (SVM) tools that are useful for $\mathrm{BCl}$ applications. Furthermore, calculation and visualization tools are provided that enable neurofeedback applications.

The implemented incremental GLM, which is used in real-time fMRI applications since many years, could potentially be used as a verification of participant's performance and speed up the experiments by giving more insights during the experiment. This would allow, for example, to stop a localizer experiment earlier in case that a required performance level is not reached saving time that can be used to better instruct the participant. A standard (windowed) GLM is used to estimate single-trial channel responses as part of the classification tools. The (incremental) GLM can also be used to regress out confounds (e.g. drifts) from the data providing cleaned input data for neurofeedback and classification sub-routines. The high sampling frequencies used in fNIRS experiments introduce a significant temporal autocorrelation within the time course which can lead to inaccurate estimation of the signal degrees of freedom and error covariance using an OLS based algorithms(Barker, Aarabi, \& Huppert, 2013). Therefore, the current version of Turbo-Satori reports beta-values as a default output. Nevertheless there are popular methods to mitigate the effect include data "pre-coloring"(YE, TAK, JANG, JUNG, \& JANG, 2009) and "pre-whitening"(Barker et al., 2013). Especially, in a recent paper(Barker et al., 2016) Barker et al. presented an online least square estimation algorithm incorporating the "prewhitening" technique for real time fNIRS. These tools are planned to be integrated in a future version of Turbo-Satori.

While Turbo-Satori already offers many relevant features, further improvements and integration of new tools for real-time fNIRS processing and analysis is needed. To allow adding custom calculations, 
a plugin interface has been created enabling software developers to extend the capabilities of the software.

The broad spectrum of different kinds of IIR filters offer a high flexibility for the researcher to easily select a good filter and corresponding settings during data acquisition for each individual participant. As shown in (Naseer \& Hong, 2015) these individual filtering can be a crucial factor if the data quality is maybe not optimal and very noisy.

The sampling rate of fNIRS data acquisition can be selected in the hardware setup of the NIRx NIRs device. It allows to freely decide based on the number of sources, detectors and steps, how fast the data should be acquired. For real-time fNIRS the sampling frequency is in principle the maximum time that is available to still perform the analysis and preprocessing in real-time. The calculation time strongly depends on the number of channels used in the analysis. Using only 20 channels resulted in a much faster calculation allowing for an overall higher sampling rate in the future. Using only the channels of interest in the data analysis would therefore be the best option for real-time applications whereas for the localizer online analysis all channels could be considered, ensuring the best selection for the $\mathrm{BCl}$ or neurofeedback experiment.

Also in the field of neurofeedback based treatments fNIRS gains more attention and more studies are recently published(Kober et al., 2014; Marx et al., 2015; Mihara \& Miyai, 2016). This shows the importance and need of more tools for real-time processing which will allow more researches to focus their research on real-time fNIRS applications and treatments as it was the case for real-time fMRI studies(Sulzer et al., 2013). The standardization of the accessibility of fNIRS data is also very important to facilitate the applications of real-time fNIRS experiments using different hardware to record the fNIRS signal. In this article, we based our developments on hardware from NIRx who use a proprietary interface to gain access to the fNIRS data in real-time. An alternative would be the use of an already elaborated library like the lab streaming layer (LSL)("Lab streaming layer (LSL)," n.d.) for the unified collection of measurement time series in research experiments. One can gain access to the software in a collaborative environment since new tools and features are continuously added. Also, it is possible that the software may become commercially available in the future. 


\section{CONCLUSION}

We presented Turbo-Satori a feature rich software toolbox for real-time fNIRS neurofeedback and $\mathrm{BCl}$ applications. An example setup was proposed using a clear differentiation between data acquisition, processing and presentation ensuring a good workflow and straightforward interaction with the different tools. With data from four experiments we showed the stability and reliability of the calculation performance from preprocessing and analysis methods ensuring a durable performance even for very long experiments running for several hours. 


\section{ACKNOWLEDGMENTS}

We thank Amaia Benitez, Hannah Boeijkens, Laurien Nagels-Coune and especially Bettina Sorger for their valuable feedback during the development of the software. We also want to thank Stefan Debener and Ling Chia-Chen who perform the first neurofeedback study using Turbo-Satori. This research was financially supported by the European Commission's Health Cooperation Work Programme of the 7th Framework Programme, under the Grant Agreement $n^{\circ} 602186$ (BRAINTRAIN). 


\section{REFERENCES}

A Collection of Useful C++ Classes for Digital Signal Processing. (n.d.). Retrieved April 7, 2017, from https://github.com/vinniefalco/DSPFilters

Antoniou, A. (2006). Digital Signal Processing: Signals, Systems, and Filters. McGraw Hill. McGraw-Hill. https://doi.org/10.1036/0071454241

Barker, J. W., Aarabi, A., \& Huppert, T. J. (2013). Autoregressive model based algorithm for correcting motion and serially correlated errors in fNIRS. Biomedical Optics Express, 4(8), 1366-1379. https://doi.org/10.1364/BOE.4.001366

Barker, J. W., Rosso, A. L., Sparto, P. J., \& Huppert, T. J. (2016). Correction of motion artifacts and serial correlations for real-time functional nearinfrared spectroscopy. Neurophotonics, 3(3), 31410. https://doi.org/10.1117/1.NPh.3.3.031410

Boas, D. A., Strangman, G., Culver, J. P., Hoge, R. D., Jasdzewski, G., Poldrack, R. A., ... Mandeville, J. B. (2003). Can the cerebral metabolic rate of oxygen be estimated with near-infrared spectroscopy? Physics in Medicine and Biology, 48(15), 2405-2418. Retrieved from http://www.ncbi.nlm.nih.gov/pubmed/12953906

BrainStim. (n.d.). Retrieved April 7, 2017, from https://svengijsen.github.io/BrainStim/

Chang, C., \& Lin, C. (2013). LIBSVM : A Library for Support Vector Machines. ACM Transactions on Intelligent Systems and Technology (TIST), 2, 1-39. https://doi.org/10.1145/1961189.1961199

Cui, X., Bray, S., \& Reiss, A. L. (2010). Functional near infrared spectroscopy (NIRS) signal improvement based on negative correlation between oxygenated and deoxygenated hemoglobin dynamics. Neurolmage, 49(4), 3039-3046. https://doi.org/10.1016/j.neuroimage.2009.11.050

Delpy, D. T., Cope, M., Zee, P. van der, Arridge, S., Wray, S., \& Wyatt, J. (1988). Estimation of optical pathlength through tissue from direct time of flight measurement. Physics in Medicine and Biology, 33(12), 1433-1442. https://doi.org/10.1088/0031-9155/33/12/008

Essenpreis, M., Cope, M., Elwell, C. E., Arridge, S. R., van der Zee, P., \& Delpy, D. T. (1993). Wavelength dependence of the differential pathlength factor and the log slope in time-resolved tissue spectroscopy. Advances in Experimental Medicine and Biology, 333, 9-20. https://doi.org/10.1007/978-1-4899-2468-1_2

Girouard, A. (2010). Towards adaptive user interfaces using real time fNIRS. ProQuest Dissertations and Theses, 3422034(August), 201. Retrieved from

http://ezproxy.net.ucf.edu/login?url=http://search.proquest.com/docvie w/751927102 ?accountid=10003\%5Cnhttp://sfx.fcla.edu/ucf?url_ver=Z39 
$.88-$

2004\&rft_val_fmt=info:ofi/fmt:kev:mtx:dissertation\&genre=dissertations $+\&+$ theses \&sid=ProQ:ProQuest+Dissertations $+\&+T$

Guennebaud, G., Jacob, B., \& others. (n.d.). C++ template library for linear algebra: matrices, vectors, numerical solvers, and related algorithms. Retrieved April 7, 2017, from http://eigen.tuxfamily.org

Kober, S. E., Wood, G., Kurzmann, J., Friedrich, E. V. C., Stangl, M., Wippel, T., ... Neuper, C. (2014). Near-infrared spectroscopy based neurofeedback training increases specific motor imagery related cortical activation compared to sham feedback. Biological Psychology, 95, 21-30. https://doi.org/10.1016/j.biopsycho.2013.05.005

Krause, F., Benjamins, C., Lührs, M., Eck, J., Noirhomme, Q., Rosenke, M., ... Goebel, R. (2017). Real-time fMRI-based self-regulation of brain activation across different visual feedback presentations. Brain-Computer Interfaces, $4(1-2)$,

87-101. https://doi.org/10.1080/2326263X.2017.1307096

Krause, F., \& Lindemann, O. (2014). Expyriment: A Python library for cognitive and neuroscientific experiments. Behavior Research Methods, 46(2), 416428. https://doi.org/10.3758/s13428-013-0390-6

Lab streaming layer (LSL). (n.d.). Retrieved April 7, 2017, from https://github.com/sccn/labstreaminglayer

Lorenz, R., Pascual, J., Blankertz, B., \& Vidaurre, C. (2014). Towards a holistic assessment of the user experience with hybrid BCls. Journal of Neural Engineering, 11(3), 35007. Retrieved from http://stacks.iop.org/17412552/11/i=3/a=035007

Lührs, M., Sorger, B., Goebel, R., \& Esposito, F. (2017). Automated selection of brain regions for real-time $\mathrm{fMRI}$ brain-computer interfaces. Journal of Neural Engineering, 14(1), 16004. https://doi.org/10.1088/17412560/14/1/016004

Marx, A.-M., Ehlis, A.-C., Furdea, A., Holtmann, M., Banaschewski, T., Brandeis, D., ... Strehl, U. (2015). Near-infrared spectroscopy (NIRS) neurofeedback as a treatment for children with attention deficit hyperactivity disorder (ADHD) a pilot study. Frontiers in Human Neuroscience, 8, 1038. https://doi.org/10.3389/fnhum.2014.01038

Mihara, M., \& Miyai, I. (2016). Review of functional near-infrared spectroscopy in neurorehabilitation. Neurophotonics, 3(3), 31414. https://doi.org/10.1117/1.NPh.3.3.031414

Naci, L., Cusack, R., Jia, V. Z., \& Owen, A. M. (2013). The brain's silent messenger: using selective attention to decode human thought for brainbased communication. J Neurosci, 33(22), 9385-9393. https://doi.org/10.1523/jneurosci.5577-12.2013

Naseer, N., \& Hong, K.-S. (2015). fNIRS-based brain-computer interfaces: a 
review. Frontiers in Human Neuroscience, 9(JAN), 3. https://doi.org/10.3389/fnhum.2015.00003

NIRx support website. (n.d.). Retrieved April 7, 2017, from http://support.nirx.de/downloads/

Ogawa, S., Lee, T. M., Kay, A. R., \& Tank, D. W. (1990). Brain magnetic resonance imaging with contrast dependent on blood oxygenation. Proceedings of the National Academy of Sciences of the United States of America, 87(24), 9868-9872. Retrieved from http://www.ncbi.nlm.nih.gov/pubmed/2124706

Pollock, D. S. G. (1999). Handbook of time series analysis, signal processing, and dynamics. Signal processing and its applications.

Popescu, T. D. (1996). Introduction to signal processing. Control Engineering Practice, 4(12), 1771-1772. https://doi.org/10.1016/S09670661(96)90009-X

Qt. (n.d.). Multiscreen / cross platform application development with Qt. Retrieved April 7, 2017, from https://www.qt.io/qt-for-applicationdevelopment/

Sato, J. R., Basilio, R., Paiva, F. F., Garrido, G. J., Bramati, I. E., Bado, P., ... Moll, J. (2013). Real-time $\mathrm{fMRI}$ pattern decoding and neurofeedback using FRIEND: An FSL-integrated $\mathrm{BCl}$ toolbox. PLOS ONE, 8(12), 1-13. https://doi.org/10.1371/journal.pone.0081658

Solovey, E. T. (2012). Real-Time FNIRS Brain Input For Enhancing Interactive Systems. ProQuest Dissertations and Theses, 3512420, 131. Retrieved from

http://ezproxy.net.ucf.edu/login?url=http://search.proquest.com/docvie w/1026792625?accountid=10003\%5Cnhttp://sfx.fcla.edu/ucf?url_ver=Z3 9.88-

2004\&rft_val_fmt=info:ofi/fmt:kev:mtx:dissertation\&genre=dissertations $+\&+$ theses\&sid=ProQ:ProQuest+Dissertations+\&+

Sorger, B., Dahmen, B., Reithler, J., Gosseries, O., Maudoux, A., Laureys, S., \& Goebel, R. (2009). Another kind of "BOLD Response": answering multiplechoice questions via online decoded single-trial brain signals. Progress in Brain Research, 177(C), 275-292. https://doi.org/10.1016/S00796123(09)17719-1

Sorger, B., Reithler, J., Dahmen, B., \& Goebel, R. (2012). A real-time fMRI-based spelling device immediately enabling robust motor-independent communication. Current Biology, 22(14), 1333-1338. https://doi.org/10.1016/j.cub.2012.05.022

Sulzer, J., Haller, S., Scharnowski, F., Weiskopf, N., Birbaumer, N., Blefari, M. L., ... Sitaram, R. (2013). Real-time fMRI neurofeedback: Progress and challenges. Neurolmage, 76, 386-399. https://doi.org/10.1016/j.neuroimage.2013.03.033 
W. B. Gratzer. (n.d.). Tabulated Molar Extinction Coefficient for Hemoglobin. Retrieved July 14, 2017, from http://omlc.ogi.edu/spectra/hemoglobin/summary.html

Weiskopf, N., Veit, R., Erb, M., Mathiak, K., Grodd, W., Goebel, R., \& Birbaumer, N. (2003). Physiological self-regulation of regional brain activity using real-time functional magnetic resonance imaging (fMRI): Methodology and exemplary data. Neurolmage, 19(3), 577-586. https://doi.org/10.1016/S1053-8119(03)00145-9

YE, J., TAK, S., JANG, K., JUNG, J., \& JANG, J. (2009). NIRS-SPM: Statistical parametric mapping for near-infrared spectroscopy. Neurolmage, 44(2), 428-447. https://doi.org/10.1016/j.neuroimage.2008.08.036 


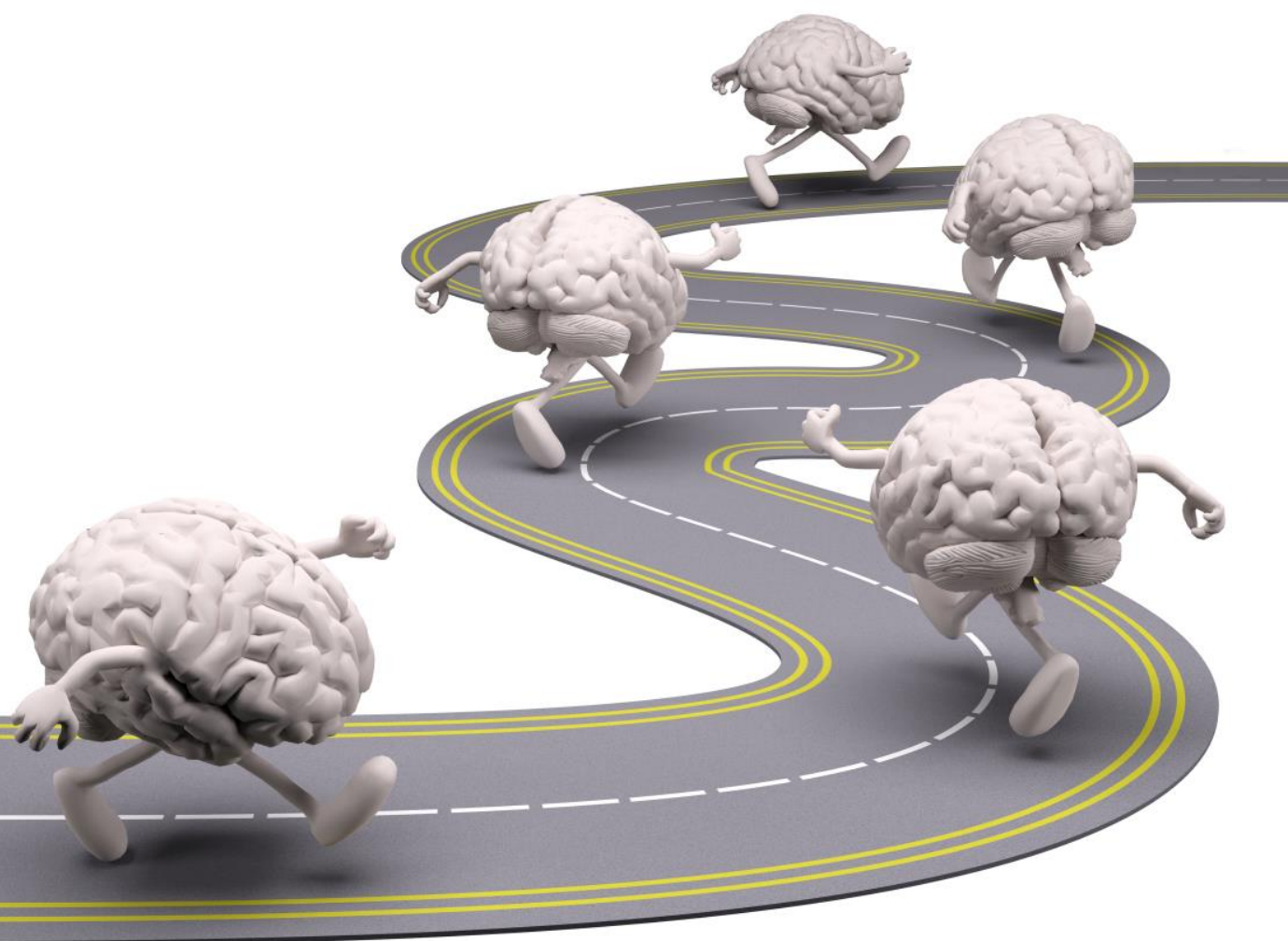




\section{Chapter 5}

Summary, general discussion and conclusion 


\section{SUMMARY}

The focus of this thesis was to create and advance methods for real-time $\mathrm{BCl}$ applications for two functional brain-imaging modalities, $\mathrm{fMRI}$ and fNIRS, both relying on the hemodynamic brain response. First the automatized selection of target areas for rt-fMRI $\mathrm{BCl}$ applications was described followed by the technological advancements in MR-image acquisition allowing for more sensitive and specific neurofeedback presentations, and at the end the mobile $\mathrm{BCl}$ applications using TurboSatori and fNIRS - a method which also has high temporal resolution.

Chapter 2 described two methods to automatize the selection of ROls for $\mathrm{BCl}$ applications. Even though the manual expert selection still achieved better accuracies as compared to automatic selection, future developments may close this gap, e.g., by adding real-time adaptations to the ROI selection, and allow for fully automatized procedures were the $\mathrm{BCl}$ operator only has a quality-control and participant-handling task.

Chapter 3 shaded light on the technological advancements in fMRI neuroimaging methods allowing for much shorter TRs. It was shown that there is a clear benefit of using very short TRs, namely the potential to explain more variance with respect to the known sources contributing to the MR signal and a clearer picture of the underling hemodynamic couplings and the HRF. This is especially important for neurofeedback applications allowing to control for BOLD changes artificially induced by physiological adaptations (conscious or unconscious).

The last chapter focused on applying high-sampling rates in mobile applications, that would allow performing experiments, for example, investigating real-world social interactions, in which free movement is a substantial element (e.g., playing an instrument or doing sports), which are not possible to conduct in an MRI environment because participants need to lie down and cannot move. A software toolbox called TurboSatori was developed and introduced, serving as the real-time analysis software for fNIRS data allowing performing real-time fNIRS $\mathrm{BCl}$ and Neurofeedback experiments. 


\section{GENERAL DISCUSSION}

On the first sight, the chapters of this thesis may not seem to be directly connected. However, the insights from each individual finding helped already or might help in the future to improve the development of the later applications. For example, the automatic ROI selection can be easily transferred to fNIRS and would allow objectifying the selection of channels for $\mathrm{BCl}$ experiments. Also, the results of chapter 3 allows near real-time AR corrected GLM analysis for fNIRS applications and have already yielded into adaptations in the developed fNIRS software. Especially the reduction of autocorrelation in the GLM residuals by adding more informing confound predictors is very important. Combining different modalities and knowledge transfer to create new applications is therefore a critical aspect in the development of the rt-fMRI and rt-fNIRS. Importantly, both $\mathrm{FMRI}$ and fNIRS hold the potential to become available as treatment tools in a clinical context. This potential is also the basis for the long-term goal of this thesis so that each chapter possibly support this path. Research in clinical sub populations has already shown that fMRINF can be effective (Thibault, MacPherson, Lifshitz, Roth, \& Raz, 2018). However, in order to provide rt-fMRI NF to patients, the costs for fMRI sessions need to be fully or partially covered by health insurances. A successful treatment could support the development of more costeffective tools to support patients, e.g., to control their craving (Cox et al., 2016). The cost-effectiveness is hereby a crucial factor and a cheaper rt-NF treatment method would be beneficial to employ neurofeedback as a general therapy alternative or combination with treatment as usual. Since most hospitals have an MRI machine, the availability is a rather neglectable issue even though patients would have to visit the hospital or clinic to receive the treatment.

As for fNIRS, it has been shown that it has a high potential to be successfully used as a neurofeedback treatment tool as well, e.g., for psychiatric disorders like schizophrenic illnesses or affective disorders (Barth, Strehl, Fallgatter, \& Ehlis, 2016; Ehlis, Schneider, Dresler, \& Fallgatter, 2014; Marx et al., 2015; Mihara et al., 2012). Moreover, even if only superficial brain areas can be reached with fNIRS, there are possibilities to target parts of brain networks which are coupled with deeper areas like the amygdala or striatum. As recently shown by Intrator and colleagues (Meir-Hasson, Kinreich, Podlipsky, Hendler, \& Intrator, 
2014), it is potentially possible to also use markers from other modalities to train specific brain areas. They used EEG to create the so called fingerprints based on the combined information of $\mathrm{FMRI}$ and EEG (MeirHasson et al., 2014). After creation of these fingerprints the neurofeedback training could be performed only using EEG. With this possibility in mind, the potentials of fNIRS are still to be investigated and need further research in multiple directions.

The hardware costs could be estimated as low as of $1 / 30$ or less of the cost of an 3T MRI machine. This implies a potentially wider treatment possibility compared to rt-fMRI (Cutini \& Brigadoi, 2014). Not only that, but, as fNIRS is a mobile technique, it opens the possibility to provide treatments at home.

Looking at the literature, one can find only little efforts combining fMRI and fNIRS (Cui, Bray, Bryant, Glover, \& Reiss, 2011; Heinzel et al., 2013; Steinbrink et al., 2006; Wijeakumar, Huppert, Magnotta, Buss, \& Spencer, 2017). Mainly because the source of the fNIRS signal is currently not completely clear (Steinbrink et al., 2006). Still, the mobile aspect of fNIRS is very promising and can path the way towards real-world applications. Also vice versa the research in $\mathrm{fMRI}$ could potentially contribute much more to the development of the fNIRS field. Since both method measure hemodynamic changes, more effort should be taken (e.g., long term simultaneous recordings or combined clinical trials) to allow both fields to benefit from each other and speed up the developments especially for research targeting the treatment of specific diseases as described in the introduction of this thesis.

It becomes clear that both methods have shown strong potential for new clinical applications and therefore should also be further developed in the future, even enforcing the efforts to benefit from each other's developments and research outcomes. This can imply in the future that if it can be shown that treatment was successful using fNIRS or fMRI the other method would constitute an alternative option. Meaning that a successful regulation using rt-fMRI and a possible coverage using fNIRS could result in a combined treatment (e.g., pre- and post-MRI and rt-fMRI sessions and in between rt-fNIRS sessions). With this, the costs can be significantly reduced - keeping the insights and possible research outcomes high.

Comparing rt-fMRI with rt-fNIRS only in these points already shows that there is a need for both methods in the future. Also, because one cannot 
cover the same areas with fNIRS compared to fMRI because fNIRS cannot reach deep brain structures till now. On the other hand, can fMRI not be used in real-world environments and can therefore only be used in specifically designed situations. Also, the possibility to use fNIRS during the performance of active tasks like playing and instrument, doing sports or social interactions will become more and more important in the future (Cutini \& Brigadoi, 2014). 


\section{CONCLUSION}

In the beginning of the thesis the increase in publications on rt-fMRI and rt-fNIRS was shown. This increase is very promising but needs to be supported by more standardized methods, procedures and routines for both rt-fMRI and rt-fNIRS research and clinical applications. If there is enough effort in making these tools useable for a broad field and not only specialists, only then there will be a chance to change and improve standard treatments and help to create new possible therapies. EEG neurofeedback is a good example, which shows how a method that was used only for research, e.g., to study neurofeedback applications in ADHD, anxiety, depression, epilepsy, autistic spectrum disorder, insomnia, drug addiction, schizophrenia, learning disabilities, dyslexia and dyscalculia (Marzbani, Marateb, \& Mansourian, 2016), is now available as a treatment for patients suffering for example from ADHD (Micoulaud-Franchi et al., 2014). This promising development should serve as a good example for future developments of rt-fMRI and rt-fNIRS. 


\section{REFERENCES}

Barth, B., Strehl, U., Fallgatter, A. J., \& Ehlis, A.-C. (2016). Near-Infrared Spectroscopy based Neurofeedback of Prefrontal Cortex Activity: A Proofof-Concept Study. Frontiers in Human Neuroscience, 10, 633. https://doi.org/10.3389/fnhum.2016.00633

Cox, W. M., Subramanian, L., Linden, D. E. J., Lührs, M., McNamara, R., Playle, R., ... Ihssen, N. (2016). Neurofeedback training for alcohol dependence versus treatment as usual: study protocol for a randomized controlled trial. Trials, 17(1), 480. https://doi.org/10.1186/s13063-016-1607-7

Cui, X., Bray, S., Bryant, D. M., Glover, G. H., \& Reiss, A. L. (2011). A quantitative comparison of NIRS and fMRI across multiple cognitive tasks. Neurolmage, 54(4), 2808-2821. https://doi.org/10.1016/j.neuroimage.2010.10.069

Cutini, S., \& Brigadoi, S. (2014). Unleashing the future potential of functional near-infrared spectroscopy in brain sciences. Journal of Neuroscience Methods, 232, 152-156. https://doi.org/10.1016/j.jneumeth.2014.05.024

Ehlis, A. C., Schneider, S., Dresler, T., \& Fallgatter, A. J. (2014). Application of functional near-infrared spectroscopy in psychiatry. Neurolmage, 85, 478488. https://doi.org/10.1016/j.neuroimage.2013.03.067

Heinzel, S., Haeussinger, F. B., Hahn, T., Ehlis, A. C., Plichta, M. M., \& Fallgatter, A. J. (2013). Variability of (functional) hemodynamics as measured with simultaneous fNIRS and fMRI during intertemporal choice. Neurolmage, 71, 125-134. https://doi.org/10.1016/j.neuroimage.2012.12.074

Marx, A.-M., Ehlis, A.-C., Furdea, A., Holtmann, M., Banaschewski, T., Brandeis, D., ... Strehl, U. (2015). Near-infrared spectroscopy (NIRS) neurofeedback as a treatment for children with attention deficit hyperactivity disorder (ADHD)â€"a pilot study. Frontiers in Human Neuroscience, 8, 1038. https://doi.org/10.3389/fnhum.2014.01038

Marzbani, H., Marateb, H. R., \& Mansourian, M. (2016). Neurofeedback: A Comprehensive Review on System Design, Methodology and Clinical Applications. Basic and Clinical Neuroscience, 7(2), 143-158. https://doi.org/10.15412/J.BCN.03070208

Meir-Hasson, Y., Kinreich, S., Podlipsky, I., Hendler, T., \& Intrator, N. (2014). An EEG Finger-Print of fMRI deep regional activation. Neurolmage, 102(P1), 128-141. https://doi.org/10.1016/j.neuroimage.2013.11.004

Micoulaud-Franchi, J.-A., Geoffroy, P. A., Fond, G., Lopez, R., Bioulac, S., \& Philip, P. (2014). EEG neurofeedback treatments in children with ADHD: an updated meta-analysis of randomized controlled trials. Frontiers in Human Neuroscience, 8, 906. https://doi.org/10.3389/fnhum.2014.00906

Mihara, M., Miyai, I., Hattori, N., Hatakenaka, M., Yagura, H., Kawano, T., ... Kubota, K. (2012). Neurofeedback using real-time near-infrared 
spectroscopy enhances motor imagery related cortical activation. PLoS ONE, 7(3), e32234. https://doi.org/10.1371/journal.pone.0032234

Steinbrink, J., Villringer, A., Kempf, F., Haux, D., Boden, S., \& Obrig, H. (2006). Illuminating the BOLD signal: combined fMRI-fNIRS studies. Magnetic Resonance Imaging, 24(4), 495-505. https://doi.org/10.1016/j.mri.2005.12.034

Thibault, R. T., MacPherson, A., Lifshitz, M., Roth, R. R., \& Raz, A. Neurofeedback with fMRI: A critical systematic review, 172 Neurolmage $\S$ (2018). Academic Press. https://doi.org/10.1016/j.neuroimage.2017.12.071

Wijeakumar, S., Huppert, T. J., Magnotta, V. A., Buss, A. T., \& Spencer, J. P. (2017). Validating an image-based fNIRS approach with $\mathrm{fMRI}$ and a working memory task. Neurolmage, 147, 204-218. https://doi.org/10.1016/j.neuroimage.2016.12.007 



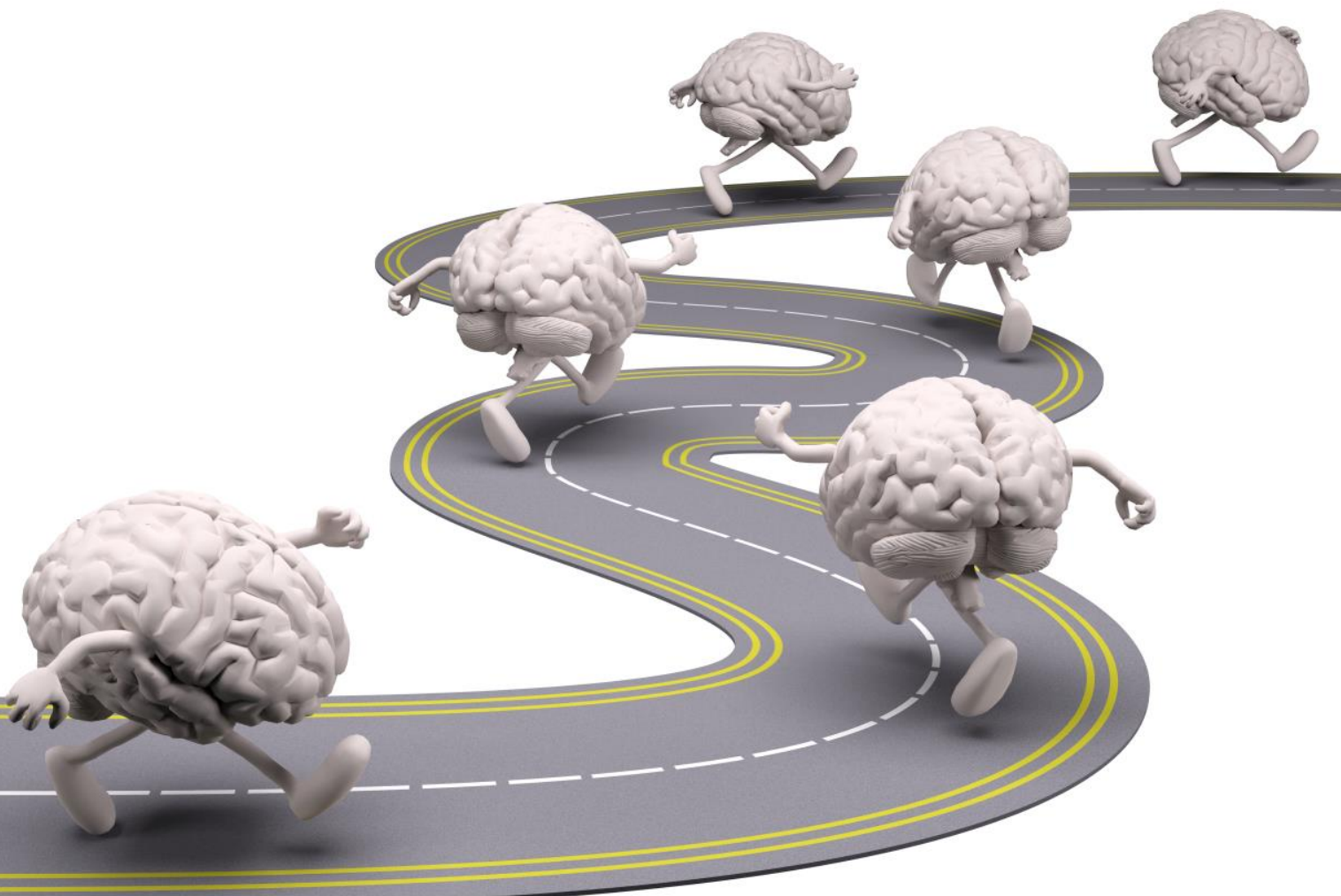




\section{ADDENDUM}

Knowledge valorization

Acknowledgments

Curriculum Vitae

Publications 



\section{Knowledge valorization}

In this chapter, the worth of the gained knowledge from this thesis is evaluated concerning a new possible value that can be created based on the presented findings. Examples for these possible values are very diverse and range from patents, licenses, open source tools, algorithms and software to many more. We will point out the direct and indirect merit of this thesis concerning the developed methods and software, starting with the potential benefits for a more widespread application of neurofeedback treatments. The different chapters will be targeted individually, and the relevant points, target groups, activities/products, innovation, and implementation will be discussed.

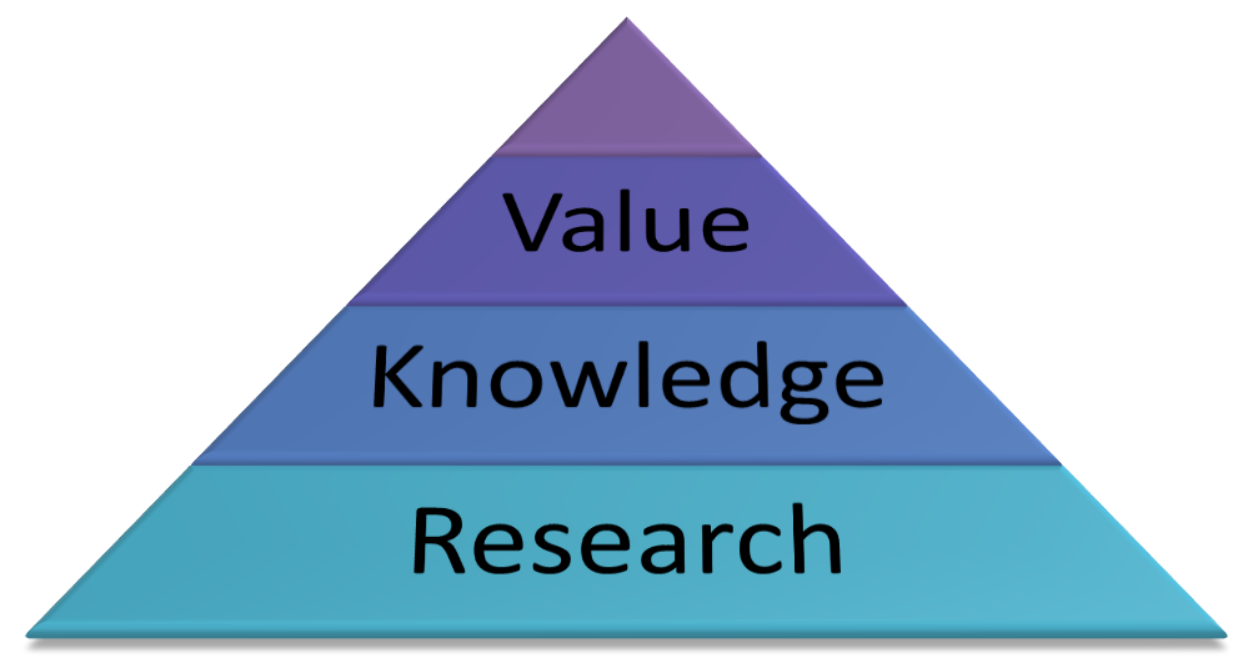




\section{Potential knowledge valorization from chapter 2}

As already described in the introduction of this thesis, the increasing amount of publications in the field of $\mathrm{BCl}$ and especially neurofeedback research shows the increasing interest and the potential of these approaches (Mihara \& Miyai, 2016; Naseer \& Hong, 2015; Thibault, MacPherson, Lifshitz, Roth, \& Raz, 2018). Since the findings of this research are continuously becoming more sophisticated and accepted in the field, the next step should be striving to make NF therapy available to patients. To be able to provide these rather complicated methods as a therapy option, pipelines that allow a streamlined and patient-centered approach needs to be developed.

The presented findings in this chapter are of interest for clinical use in the near future (Marzbani, Marateb, \& Mansourian, 2016). With the newly developed algorithm to automatically select brain areas based on an individual localizer of the participant, we not only provide a tool that could be a part of these pipelines (Lührs, Sorger, Goebel, \& Esposito, 2017), but we also aim at reducing the need for highly trained personnel to carry out the NF therapy, and thus, making the approach simpler and easier to implement.

The products or processes, into which the research can be translated to, were already described in the chapter We based our research on the analyzed $\mathrm{fMRI}$ data from Turbo-BrainVoyager. The developed procedures were implemented entirely or partially as plugins for this product, giving a direct value to researchers using this product. The research showed that combining different routines and procedures creates a new method with a wide application range. Since it has already been implemented in a commercial product, part of this research is directly available. 


\section{Potential knowledge valorization from chapter 3}

Improving and developing new methods has always been a significant research outcome, which translates into social and economic value (Fine, Denzin, \& Lincoln, 1995). The increasing developments in MR-sequences could provide benefits for $\mathrm{fMRI}$ neurofeedback, as described in chapter three of this thesis (Assländer et al., 2013; Zahneisen et al., 2011). Investigating them does not hold a direct social or economic value, but it can translate into it, as it will bring us closer to developing efficient fMRIbased NF therapies in the near future. These do benefit not only the academic community but also the same target groups, as described in the previous section.

Concerning innovation, this chapter focused primarily on the verification of available methods suitable for the used MR-sequence. Therefore, the leading innovation was the sequence itself, and to a lesser extent, the proof of principle to use established fMRI analysis methods for these data. Next steps will be focused on implementing real-time workflows and will eventually become the most important outcome of this chapter because it will make this technique available to a broader range of applications.

\section{Potential knowledge valorization from chapter 4}

The knowledge we gained was directly transferred into the developed software for rt-fNIRS analysis, Turbo-Satori (Lührs \& Goebel, 2017). Combining the knowledge into software that gives access to very advanced procedures and algorithms in a natural way is one of the most valuable outcomes of this thesis.

Future research and developments can now be directly translated into the software package, which will further gain value over time. So, in addition to the research interest in the academic community, the software is also of interest as a tool in this community. Bringing in value from research findings to develop tools to be used for research directly. At the same time, it is essential that the tools, such as fNIRS, become available to a broader audience. fNIRS is a mobile application, which gained more interest in the recent years, as described in the introduction of this thesis. The points raised for the fMRI methods apply to fNIRS as well, with the addition that the Turbo-Satori software is more likely to be used in therapeutic applications, due to its lower complexity and costs, compared to $\mathrm{fMRI}$. Since the software itself is the product, there is no 
translation. Since the field of rt-fNIRS applications is entirely new, the software itself is innovative and can be seen as a contribution towards a more full spread of the fNIRS method. The last point, the implementation, is also already accomplished with the first release of the software.

In summary, the focus of this thesis relied on the development and improvement of automatic procedures for $\mathrm{FMRI}$ and $\mathrm{fNIRS}$ neurofeedback applications. Developing automatic routines is a strong valorization of the gained knowledge in itself since the newly developed procedures can help to pave the way to use automatic procedures for patient treatment. Even though it will still take some time to be established, every step towards this goal is helpful and will be beneficial in the upcoming years. 


\section{REFERENCES}

Assländer, J., Zahneisen, B., Hugger, T., Reisert, M., Lee, H. L., LeVan, P., \& Hennig, J. (2013). Single shot whole brain imaging using spherical stack of spirals trajectories. Neurolmage, 73, 59-70. https://doi.org/10.1016/j.neuroimage.2013.01.065

Fine, G. A., Denzin, N., \& Lincoln, Y. (1995). Handbook of Qualitative Research. Contemporary Sociology, 24(3), 416. https://doi.org/10.2307/2076550

Lührs, M., \& Goebel, R. (2017). Turbo-Satori: a neurofeedback and brain-computer interface toolbox for real-time functional near-infrared spectroscopy. Neurophotonics, 4(04), 1. https://doi.org/10.1117/1.NPh.4.4.041504

Lührs, M., Sorger, B., Goebel, R., \& Esposito, F. (2017). Automated selection of brain regions for real-time $\mathrm{fMRI}$ brain-computer interfaces. Journal of Neural Engineering, 14(1), 016004. https://doi.org/10.1088/1741-2560/14/1/016004

Marzbani, H., Marateb, H. R., \& Mansourian, M. (2016). Neurofeedback: A Comprehensive Review on System Design, Methodology and Clinical Applications. Basic and Clinical Neuroscience, 7(2), 143-158. https://doi.org/10.15412/J.BCN.03070208

Mihara, M., \& Miyai, I. (2016). Review of functional near-infrared spectroscopy in $\begin{array}{lll}\text { neurorehabilitation. } & \text { Neurophotonics, } & 3(3),\end{array}$ https://doi.org/10.1117/1.NPh.3.3.031414

Naseer, N., \& Hong, K.-S. (2015). fNIRS-based brain-computer interfaces: a review. Frontiers in Human Neuroscience, 9(JAN), 3. https://doi.org/10.3389/fnhum.2015.00003

Thibault, R. T., MacPherson, A., Lifshitz, M., Roth, R. R., \& Raz, A. Neurofeedback with fMRI: A critical systematic review, 172 Neurolmage $\S$ (2018). Academic Press. https://doi.org/10.1016/j.neuroimage.2017.12.071

Zahneisen, B., Grotz, T., Lee, K. J., Ohlendorf, S., Reisert, M., Zaitsev, M., \& Hennig, J. (2011). Three-dimensional MR-encephalography: Fast volumetric brain imaging using rosette trajectories. Magnetic Resonance in Medicine, 65(5), 1260-1268. https://doi.org/10.1002/mrm.22711 
•・・ 


\section{Acknowledgments}

In the last couple of years, I was very fortuned getting to know so many great people which makes it now very difficult for me to not forgot anyone in this acknowledgment.

First, I would like to thank all my supervisors who made this thesis possible. Fabrizio, you helped me so much at the beginning of my Ph.D. to get started, and I'm very thankful for all the time you spend together with me working on revisions. This will accompany me for my whole research career. Bettina, we had so many productive meetings which I really appreciate because they helped me a lot to stick to my goal and succeed in this whole work. I'm looking forward to developing new BCl's and ideas together with you!

Rainer, since we first met at the HBM in Canada in 2011, I was intrigued by your never-ending enthusiasm which continued throughout my whole time here in Maastricht. I'm really thankful that you and Claudia gave me the opportunity to do a Ph.D. and I'm looking forward to working together with you on the next exciting projects. I'm also looking forward to the future trips with you Claudia, it was always nice to travel together!

To all my present and former colleagues at Brain Innovation (yes, the company has a real name, and it is not BrainVoyager (-)), thank you for the support and great time in the last six years. I really enjoyed all the activities, especially the parts including sushi! Also, the coffee break wouldn't have been so entertaining without you! Armin and Judith, thank you so much for your patience and sympathetic ear whenever I had my "short" questions and problems! But I guess this will never stop. Bianca and Hester, you are both the good souls of the company. Thank you for always cheering up with Stroopwafels and cake! Of course, I won't forget all the Mölkky afternoons with you Florian. I'm looking forward to the next projects with you! Rick, Carmine, Leon (now all part of the 30 club (-)), Caroline and Makis thanks for not forgetting to ask me for lunch even though I now most of the time bring my own food! Thank you, Anita, 
for office entertainment and your great company on the trip to Berlin and the voyages that will follow in the next years!

I also want to thank all the present and former colleagues at the $\mathrm{CN}$ department! Thank you for all the great experiences I had in the last years from projects, activities to the overall inspiring workplace. Special thanks to Amaia, Tabea, Hannah, Laurien, Valentin, Thomas, Benedikt, Judith, Sven and of course Riny and Christl for all their patients with my neverending questions and "appointment with Rainer" request. Thank you, Amaia for always listening and never stopping to ask if I'm fine, as in good. Thank you for your all the conversations Tabea! I'm really missing the thrilling car rides.

It was always important for me to have good friends that helped me not to think about work all the time! Thank you Andrè, Lea, Jonas, Sven, Dana, Nils, Jan, Martin, Christian, Sebastian, Frank, Frank, David, and Claudia. I really enjoyed all the festivals, weekends and parties and I'm looking forward to the ones that will follow (-)

At the end and most importantly, I would like to thank my family and Maria for all the continued support and the backing that allowed me to accomplish this milestone. Vielen vielen Dank für den Rückhalt und die Unterstützung! Ohne euch wäre das für mich nicht möglich gewesen! 


\section{Curriculum Vitae}

Michael Lührs was born on January $24^{\text {th }}, 1988$ in Nordhorn, Germany. In 2007, he graduated from the GBS Nordhorn Fachgymnasium Technik, Germany.

In the same year, he started studying Computational Visualistics at the Otto-von-Guericke-University in Magdeburg. He received the bachelor's degree in 2011 and continued his studies on a master's degree level in the same field. He completed his studies in 2012. The bachelor's and master's thesis focused on the improvement of software interfaces for the rt-fMRI analysis software Turbo-BrainVoyager. This lead to a proof of principle rt-fMRI hyperscanning study, which was the central point of the master thesis.

After finishing the studies, he moved to Maastricht to work as a Ph.D. candidate under the supervision of Prof. dr. Rainer Goebel, Dr. Fabrizio Esposito, and Dr. Bettina Sorger at Brain Innovation B.V. and the Department of Cognitive Neuroscience, Faculty of Psychology and Neuroscience, Maastricht University. After obtaining his Ph.D., he will continue working at Brain Innovation. 



\section{Publications}

\section{Peer-reviewed journals:}

Lührs, M., Sorger, B., Goebel, R., \& Esposito, F. (2017).

Automated selection of brain regions for real-time $\mathrm{fMRI}$ brain-computer interfaces. Journal of Neural Engineering, 14(1), 016004.

Lührs, M., \& Goebel, R. (2017).

Turbo-Satori: a neurofeedback and brain-computer interface toolbox for real-time functional near-infrared spectroscopy. Neurophotonics, 4(04), 1.

Krause, F., Benjamins, C., Lührs, M., Eck, J., Noirhomme, Q., Rosenke, M., ... Goebel, R. (2017). Real-time fMRI-based self-regulation of brain activation across different visual feedback presentations. BrainComputer Interfaces, 4(1-2), 87-101.

Neyedli, H. F., Sampaio-Baptista, C., Kirkman, M. A., Havard, D., Lührs, M., Ramsden, K., ... Johansen-Berg, H. (2018). Increasing Lateralized Motor Activity in Younger and Older Adults using Real-time fMRI during Executed Movements. Neuroscience, 378, 165-174.

Spetter, M. S., Malekshahi, R., Birbaumer, N., Lührs, M., van der Veer, A. H., Scheffler, K., ... Hallschmid, M. (2017). Volitional regulation of brain responses to food stimuli in overweight and obese subjects: A real-time fMRI feedback study. Appetite, 112, 188-195.

Cox, W. M., Subramanian, L., Linden, D. E. J., Lührs, M., McNamara, R., Playle, R., ... Ihssen, N. (2016). Neurofeedback training for alcohol dependence versus treatment as usual: study protocol for a randomized controlled trial. Trials, 17(1), 480.

Mehler, D. M. A., Sokunbi, M. O., Habes, I., Barawi, K., Subramanian, L., Range, M., Evans, J., Hood, K., Lührs, M., ... Linden, D. E. J. (2018). Targeting the affective brain - a randomized controlled trial of real-time fMRI neurofeedback in patients with depression. Neuropsychopharmacology, 1.

Mueller, C., Lührs, M., Baecke, S., Adolf, D., Luetzkendorf, R., Luchtmann, M., \& Bernarding, J. (2012). Building virtual reality fMRI paradigms: A framework for presenting immersive virtual environments. Journal of Neuroscience Methods, 209(2), 290-298. 


\section{In preparation:}

Lührs, M., Riemenschneider, B., Eck, J., Benitez, A., Poser, B., Heinecke, A., Krause, F., Esposito, F., Sorger, B., Hennig, J., Goebel, R. (2018, Neurolmage, submitted). MR-Encephalography for $\mathrm{BCl} /$ neurofeedback applications with high temporal resolution.

Kamp, T., Sorger, B., Lührs, M., Goebel, R. (2018, Neurolmage, submitted).

Gradual self-regulation of the default mode network with real-time fMRI neurofeedback.

Zich, C., Haller, S., Lührs, M., Lisk, S., Lau, J., Cohen Kadosh, K.

(2018, Cerebral Cortex, submitted). Modulatory effects of dynamic fMRI-based neurofeedback on emotion regulation networks during adolescence

Preissl, H., Kohl, S., Veit, R., Spetter, M., Günther, A., Lührs, M., ... Hallschmid, M., (2018, Neurolmage, submitted). Real-time fMRI neurofeedback training to improve eating behavior by self-regulation of the dorsolateral prefrontal cortex: a randomized controlled trial in overweight and obese subjects.

Rosenke, M., Eck, J., Noirhomme, Q., Krause, F., Benjamins, C., Lührs, M., Goebel, R. (2018, Brain-Computer-Interfaces, submitted). Selfregulation of a functional network using network connectivity and network activation during real-time fMRI.

Krause, F., Benjamins, C., Eck, J., Lührs, M., van Hof, R., Goebel,

R., (2018, HBM, submitted). Active head motion reduction in Magnetic Resonance Imaging using tactile feedback.

Zhao, Z., Yao, S., Li, K., Sindermann, C., Zhou, F., Zhao, W., Li, J., Lührs, M., Goebel, R., Kendrick, K., Becker, B. (2018, Psychotherapy \& Psychosomatics, submitted). Real-time functional connectivityinformed neurofeedback of amygdala-frontal pathways reduces anxiety

Direito, B., Lima, J., Simões, M., Sayal, A., Sousa, T., Lührs, M., Ferreira, C., Castelo-Branco, M. (2018, Neuroscience, submitted). A highly selective dynamic facial processing domain in superior temporal sulcus which can be causally used as $\mathrm{fMRI}$ neurofeedback target.

Whittaker, J., Mehler, D., Williams, A., Krause, F., Lührs, M., Wise, R., Turner, D., Linden, D. (2018, Neurolmage, submitted). The BOLD response in primary motor cortex and supplementary motor area during kinaesthetic motor imagery based gradual fMRI neurofeedback 


\section{Conference contributions:}

Lührs, M., Neyedli, H., Johansen-Berg, H., Goebel, R. (2014, HBM, Hamburg, Germany). Turbo-Feedback: A real-time fMRI neurofeedback tool using a novel network communication interface.

Lührs, M., Krause, F., Benjamins, C., Brunheim, S., Eck, J., Noirhomme, Q., Rosenke, M., Goebel, R. (2015, HBM, Hawaii, USA). Improving the workflow of real-time $\mathrm{fMRI}$ Neurofeedback experiments.

Lührs, M., Sorger, B., Goebel, R., Esposito, F., (2016, BCl Meeting, Asilomar, CA, USA). FMRI-based Letter Decoding using Real-time ICA.

Lührs, M., Goebel, R. (2016, BCI Meeting, Asilomar, CA, USA). Turbo-Satori: A novel real-time fNIRS data processing and analysis toolbox.

Lührs, M., Poser, B., Auer, T., Goebel, R. (2017, rtFIN, Nara, Japan). Retrieving $\mathrm{fMRI}$ data in real-time: difficulties and pitfalls.

Lührs, M., Riemenschneider, B., Eck, J., Benitez, A., Poser, B., Heinecke, A., Krause, F., Esposito, F., Sorger, B., Hennig, J., Goebel, R. (2017, rtFIN, Nara, Japan). Real-time MR-Encephalography for $\mathrm{BCl} /$ Neurofeedback applications.

Lührs, M., Tursic, A., Goebel, R. (2018, BCl Meeting, Asilomar, CA, USA). Functional verification of fNIRS probe locations using a generalized SVM classifier model for $\mathrm{BCl}$ applications. 
Talks:

Lührs, M., Zilverstand A. (2016, BCI Meeting, Asilomar CA, USA). Towards clinical application of $\mathrm{fMRI}$-based $\mathrm{BCls}$ and transfer to fNIRS \& EEG.

Lührs, M. (2016, fNIRS Meeting, Paris, France). A novel Neurofeedback and BCI toolbox for real-time fNIRS: Turbo-Satori.

Lührs, M. (2016, IDEA user meeting, Maastricht, Netherlands). Real-time export made easy. 